\title{
Evolution of a 4-Benzyloxy-benzylamino Chemotype to Provide Efficacious, Potent, and Isoform Selective PPARa Agonists as Leads for Retinal Disorders
}

Xiaozheng Dou, ${ }^{\dagger, \S}$ Dinesh Nath, ${ }^{\dagger,}$ Henry Shin, ${ }^{\ddagger}$ Elmar Nurmemmedov, ${ }^{\ddagger}$ Philip C. Bourne, ${ }^{\S}$ Jian-Xing Ma, ${ }^{\ddagger}$ and Adam S. Duerfeldt*i,\&

Institute for Natural Products Applications and Research Technologies and ${ }^{\S}$ Department of Chemistry \& Biochemistry, University of Oklahoma, 101 Stephenson Parkway, Stephenson Life Sciences Research Center, Norman, Oklahoma 73019, United States

Department of Physiology, University of Oklahoma Health Sciences Center, 941 Stanton L. Young Boulevard, Oklahoma City, Oklahoma 73104, United States

"John Wayne Cancer Institute \& Pacific Neuroscience Institute at Providence Saint John's Health Center, 2200 Santa Monica Boulevard, Santa Monica, California 90404, United States

Supporting information -Two-concentration luciferase data, PPAR $\alpha$ expression and purification, cellular thermal shift western blots, cytotoxicity, in silico metabolic predictions, isothermal titration calorimetry thermograms and curve fits, ${ }^{1} \mathrm{H}$ and ${ }^{13} \mathrm{C}$ NMR spectra for final compounds, HPLC traces for select leads, and pharmacokinetic data sets.

*to whom correspondence should be addressed. Department of Chemistry and Biochemistry, University of Oklahoma, 101 Stephenson Parkway, Stephenson Life Sciences Research Center, Norman, Oklahoma 73019. Phone: 1-405-325-2232. Email: adam.duerfeldt@ou.edu. 


\section{Table of Contents}

PPARa Luciferase Two-Concentration Assessment .................................................................S4

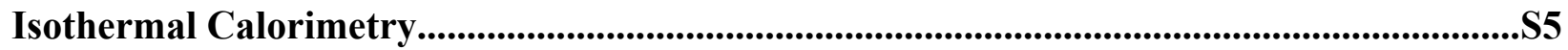

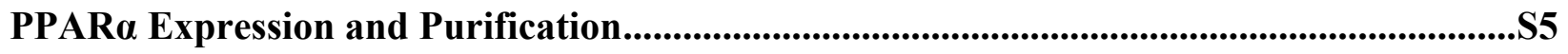

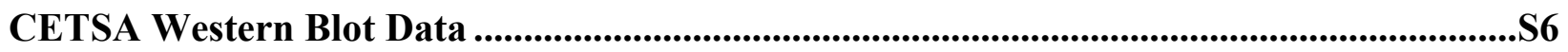

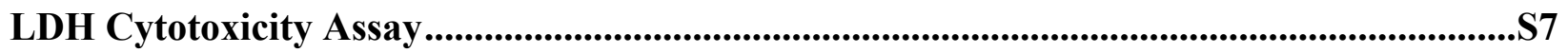

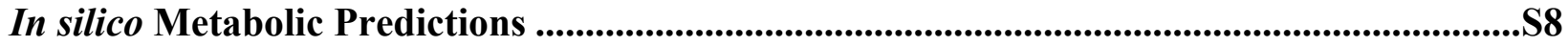

NMR Spectra for Final Compounds

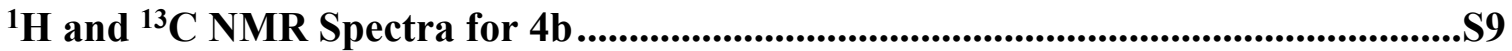

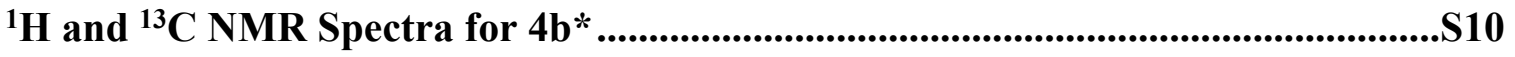

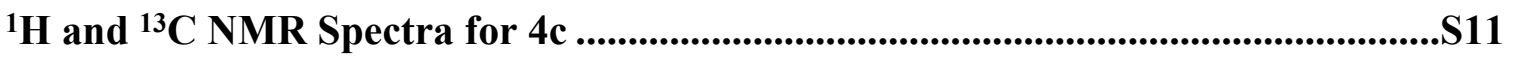

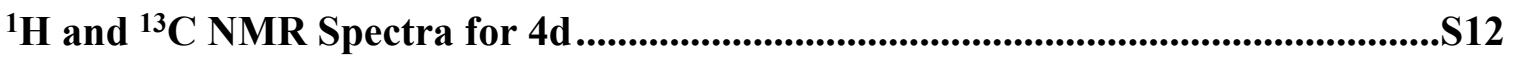

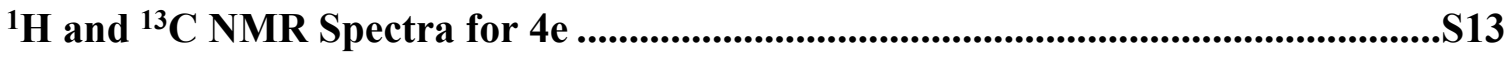

${ }^{1} \mathrm{H}$ and ${ }^{13} \mathrm{C}$ NMR Spectra for $4 \mathrm{f}$................................................................................S14

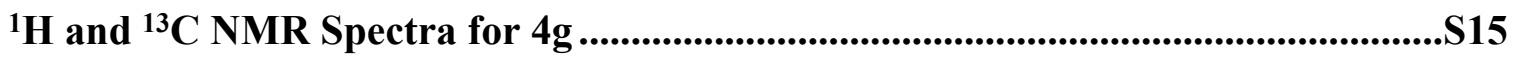

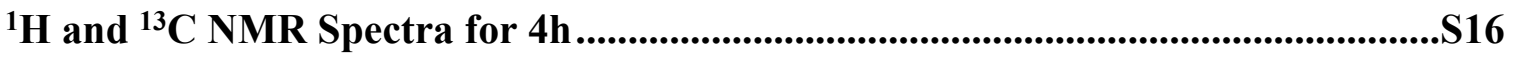

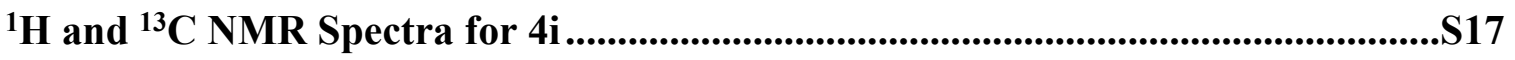

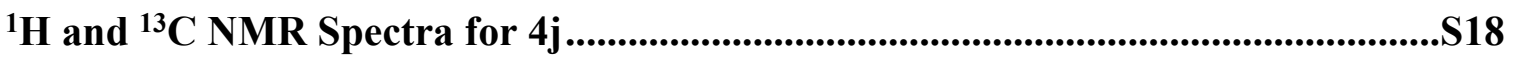

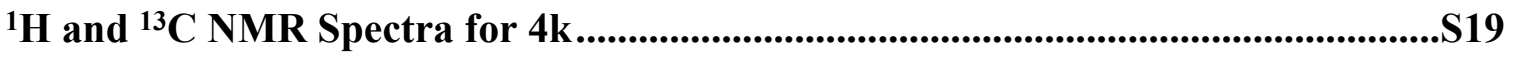

${ }^{1} \mathrm{H}$ and ${ }^{13} \mathrm{C}$ NMR Spectra for 41 .....................................................................................S20

${ }^{1} \mathrm{H}$ and ${ }^{13} \mathrm{C}$ NMR Spectra for $4 \mathrm{~m}$......................................................................................S21

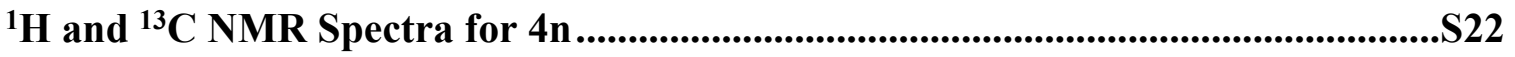

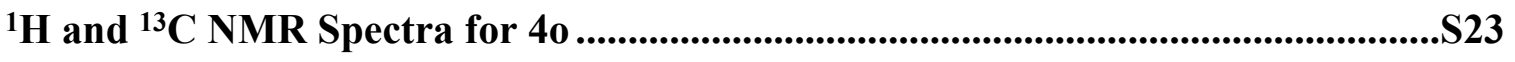

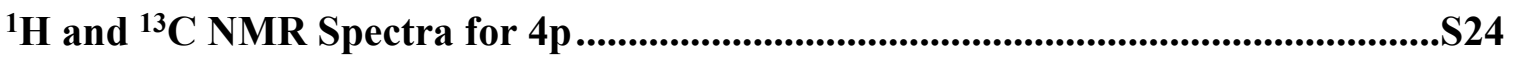

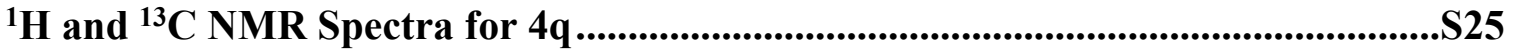

${ }^{1} \mathrm{H}$ and ${ }^{13} \mathrm{C}$ NMR Spectra for $4 \mathrm{r}$.........................................................................................S26

${ }^{1} \mathrm{H}$ and ${ }^{13} \mathrm{C}$ NMR Spectra for 4s...................................................................................S27

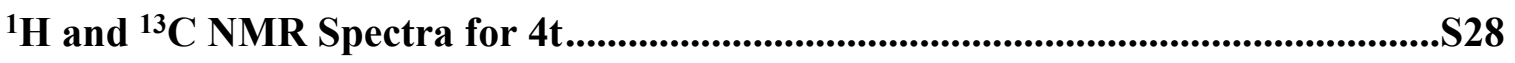

${ }^{1} \mathrm{H}$ and ${ }^{13} \mathrm{C}$ NMR Spectra for $4 \mathrm{u}$...................................................................................S29

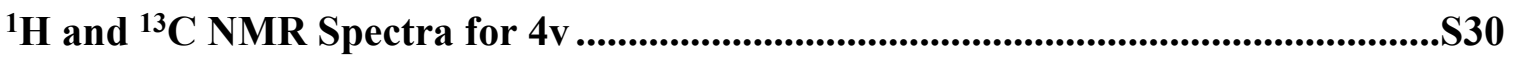

${ }^{1} \mathrm{H}$ and ${ }^{13} \mathrm{C}$ NMR Spectra for $4 \mathrm{w}$.....................................................................................S31

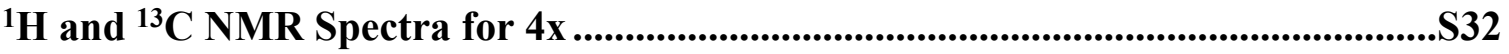




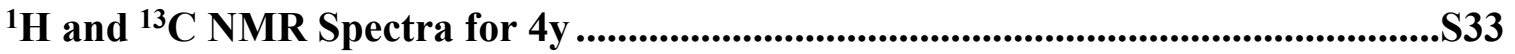

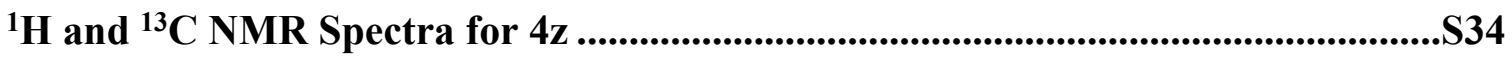

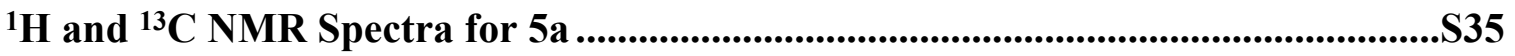

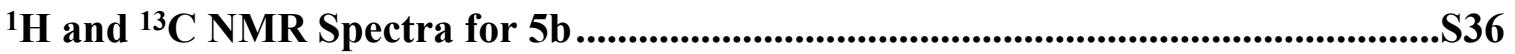

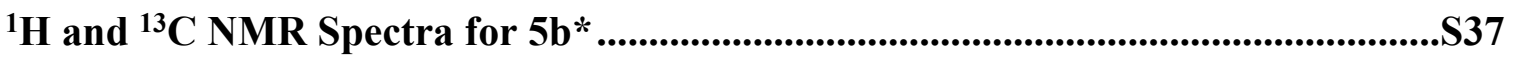

${ }^{1} \mathrm{H}$ and ${ }^{13} \mathrm{C}$ NMR Spectra for $5 \mathrm{f}$.........................................................................................

${ }^{1} \mathrm{H}$ and ${ }^{13} \mathrm{C}$ NMR Spectra for 19a .............................................................................339

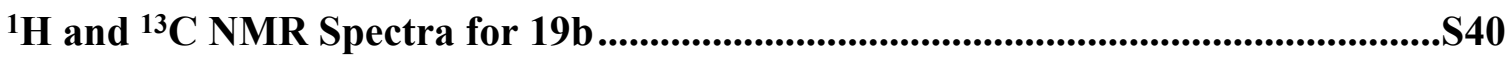

${ }^{1} \mathrm{H}$ and ${ }^{13} \mathrm{C}$ NMR Spectra for $19 \mathrm{c}$...................................................................................

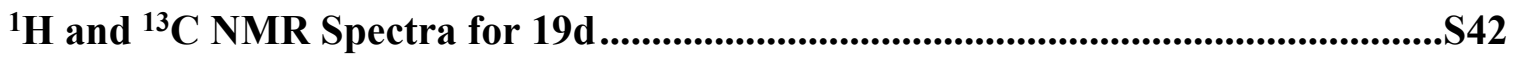

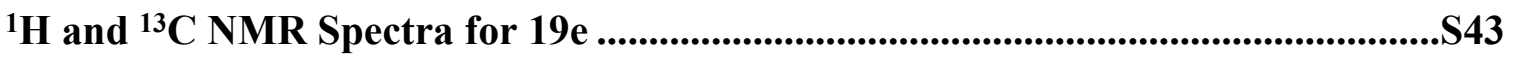

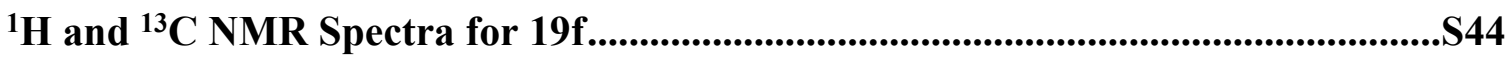

${ }^{1} \mathrm{H}$ and ${ }^{13} \mathrm{C}$ NMR Spectra for 20 ......................................................................................545

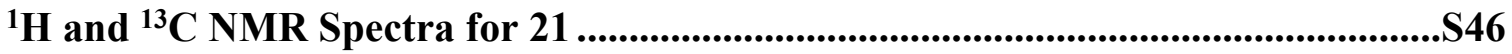

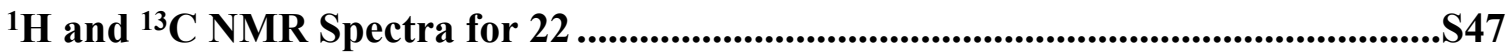

${ }^{1} \mathrm{H}$ and ${ }^{13} \mathrm{C}$ NMR Spectra for 23 ...................................................................................548

${ }^{1} \mathrm{H}$ and ${ }^{13} \mathrm{C}$ NMR Spectra for 24 ....................................................................................49

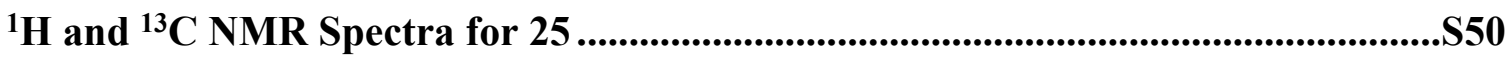

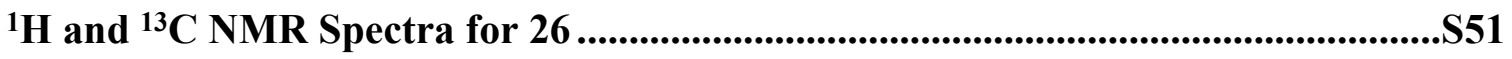

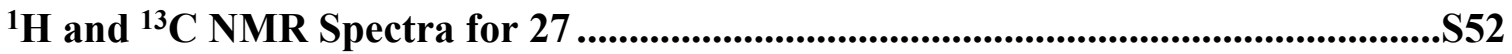

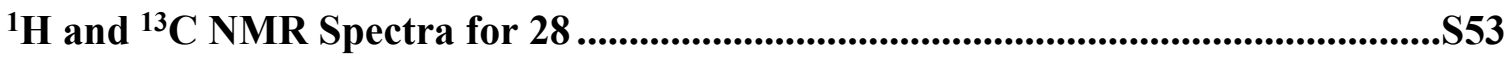

${ }^{1} \mathrm{H}$ and ${ }^{13} \mathrm{C}$ NMR Spectra for 29 ....................................................................................55

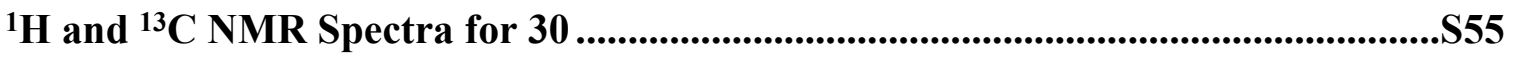

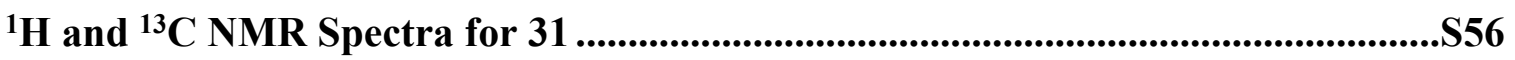

${ }^{1} \mathrm{H}$ and ${ }^{13} \mathrm{C}$ NMR Spectra for 32 ........................................................................................557

${ }^{1} \mathrm{H}$ and ${ }^{13} \mathrm{C}$ NMR Spectra for 33 .................................................................................558

HPLC Traces for Key Analogues................................................................................................59

Pharmacokinetic Data (Eurofins) .....................................................................................................S62 


\section{PPARA LUCIFERASE TWO-CONCENTRATION ASSESSMENT}

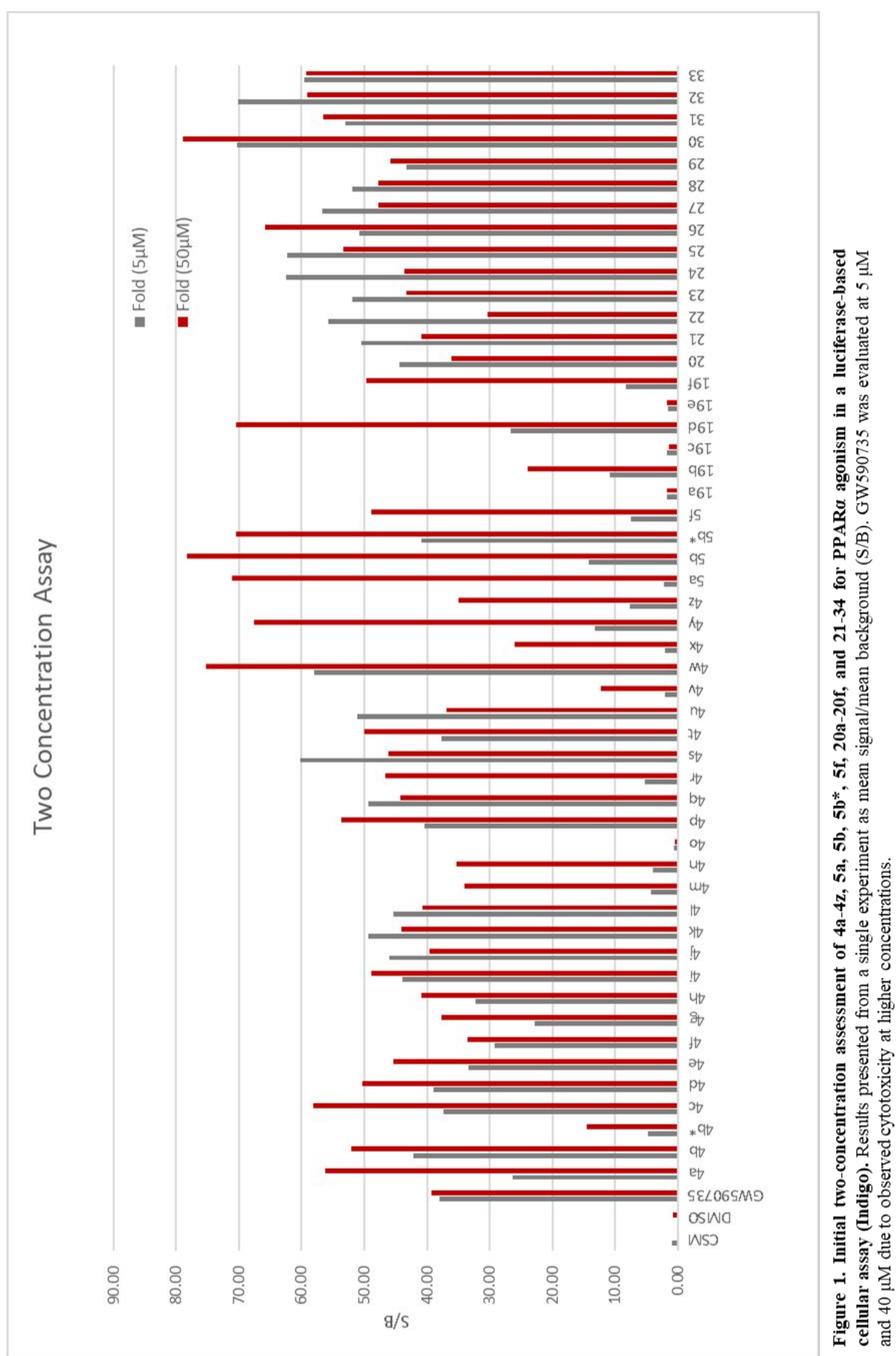




\section{ISOTHERMAL CALORIMETRY}

ITC experiments were performed using a MicroCal PEAQ ITC (MicroCal Inc., Northampton, MA, USA). PPAR $\alpha$ was concentrated down to a final concentration of $3.27 \mathrm{mg} / \mathrm{mL}$ using an Amicon Ultra-15 centrifugal concentrator (Millipore). Buffer (20 mM HEPES, $150 \mathrm{mM} \mathrm{NaCl}, \mathrm{pH} 7.4$ ) was used to dilute the ligand stock solution $(10,12.5$, or $25 \mathrm{mM}$ in DMSO). DMSO was added to the protein solution at the same percentage $(0.8-10 \%)$ of the ligand solution. Samples were centrifuged before the experiment to eliminate possible aggregates. The protein solution $(30-85 \mu \mathrm{M})$ was placed in the sample cell, and the ligand solution (2.3-15 times more concentrated than the protein) was loaded into the syringe injector. The detailed experimental parameters are shown below. The titrations involved 19 injections of $2 \mu \mathrm{L}$ at $150 \mathrm{~s}$ intervals or 13 injections of $3 \mu \mathrm{L}$ at $150 \mathrm{~s}$ intervals. The experiment was set at $25{ }^{\circ} \mathrm{C}$ and the syringe stirring speed was set at $750 \mathrm{rpm}$. Titration of ligand at same concentration into buffer with same percentage DMSO were used as a reference control. The thermodynamic data were processed with MicroCal PEAQ-ITC Analysis Software provided by Malvern. The one-site binding model was selected to calculate the value of the dissociation constant $\left(\mathrm{K}_{\mathrm{d}}\right)$, the enthalpy $(\Delta \mathrm{H})$, the binding free energy $(\Delta \mathrm{G})$, and the entropy $(-\mathrm{T} \Delta \mathrm{S})$.

\begin{tabular}{|c|c|c|c|}
\hline Ligand & {$[$ PPAR $] \mu \mathrm{M}$} & [Ligand] $\mathrm{nM}$ & DMSO $\%(\mathrm{v} / \mathrm{v})$ \\
\hline 4a (A91) & 85 & 0.2 & $0.8 \%$ \\
\hline 4b & 60 & 0.5 & $2 \%$ \\
\hline 4u & 30 & 0.25 & $2 \%$ \\
\hline GW590735 & 80 & 0.5 & $2 \%$ \\
\hline
\end{tabular}
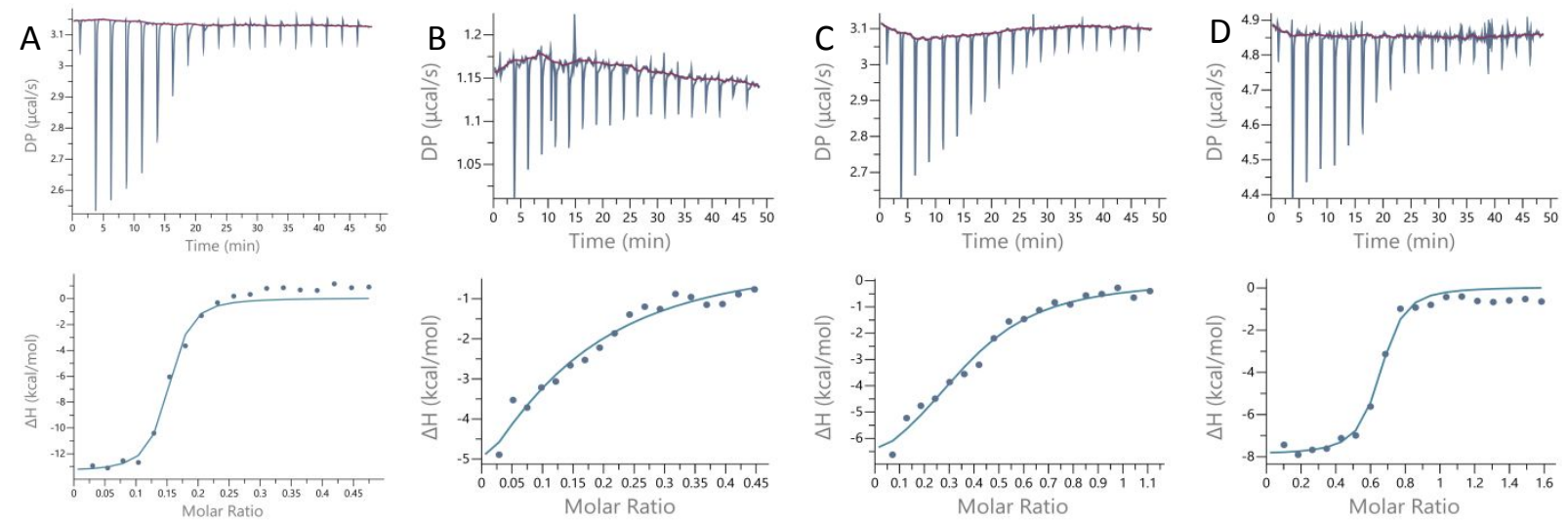

Figure S2. Isothermal titration calorimetry thermograms of select ligands to PPAR $\alpha$-LBD. The upper panel shows the raw data of a representative ITC experiment. The lower panel shows the corresponding binding isotherm fit according to the "one binding site" model. A) GW590735; B) compound 4a; C) compound 4b; and D) compound $\mathbf{4 u}$

\section{PROTEIN EXPRESSION, PURIFICATION}

Protein was expressed from the pET28c vector (Novagen) in Escherichia coli strain Rosetta ${ }^{\mathrm{TM}}$ (DE3) grown in LB media to an OD of 0.6 and then induced with $1 \mathrm{mM}$ IPTG for $20 \mathrm{~h}$ at $18^{\circ} \mathrm{C}$. Cells were harvested and resuspended in Buffer A (20 mM Tris, $150 \mathrm{mM} \mathrm{NaCl}, 5 \%$ glycerol, $5 \mathrm{mM}$ imidazole, $\mathrm{pH} 8.0$ ) at $5 \mathrm{ml} / \mathrm{g}$ pellet weight. Cultures were lysed using an Emulsiflex C-3 homogenizer (Avestin), and the lysate clarified by centrifugation $(22,000 \times \mathrm{g}, 40 \mathrm{~min})$. The supernatant was loaded onto a $5 \mathrm{ml}$ HisTrap FF Crude column (GE Life Sciences) and eluted with a linear gradient of 5-500 mM imidazole over 20 column volumes. Fractions containing PPAR $\alpha$ were identified by sodium dodecyl sulfate-polyacrylamide gel electrophoresis analysis. The His-Tag was removed by thrombin cleavage $(10 \mathrm{U}$ thrombin $/ \mathrm{mg})$ dialyzed overnight at $4{ }^{\circ} \mathrm{C}$ against buffer $\mathrm{B}(20 \mathrm{mM}$ Tris, $150 \mathrm{mM} \mathrm{NaCl}, 5 \%$ glycerol, $\mathrm{pH} \mathrm{8.0)}$. The uncleaved protein was removed by reloading the sample onto the $5 \mathrm{ml}$ HisTrap FF Crude column (GE Life Sciences). The flow through was concentrated to a final volume of $5 \mathrm{ml}$ using an Amicon Ultra-15 centrifugal concentrator (Millipore), 
and loaded on to a HiLoad Superdex 200PG size exclusion chromatography column (GE Life Sciences) pre-equilibrated with Buffer C (20 mM HEPES, $150 \mathrm{mM} \mathrm{NaCl}$, $\mathrm{pH} 7.4)$.

\section{CETSA DATA}

\section{Heat challenge, ${ }^{\circ} \mathrm{C}$}

IB: PPAR-alpha

\begin{tabular}{|c|c|c|c|c|c|c|c|c|c|}
\hline $\begin{array}{l}\overline{\tilde{D}} \\
\stackrel{0}{0} \\
\hat{N} \\
\hat{m}\end{array}$ & 웅 & $\underset{\sim}{\sim} \underset{\sim}{\sim}$ & $\stackrel{\mathscr{\sigma}}{\stackrel{g}{q}}$ & $\hat{\gamma}$ & $\begin{array}{l}0 \\
\text { N } \\
\text { N }\end{array}$ & 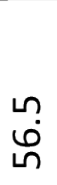 & $\begin{array}{l}\infty \\
\infty \\
\infty \\
\end{array}$ & ○. & Treatment: \\
\hline & & & 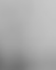 & & & & & & DMSO \\
\hline
\end{tabular}

Figure S3. Melting profile of hPPAR $\alpha$. MIO-M1 cells were subjected to a heat gradient to determine the thermal melting profile of hPPAR $\alpha$. Band intensity of remaining stable protein at each temperature was quantified, and subsequently the temperature of aggregation ( $\mathrm{T}_{\text {agg }} 50$ nd $\mathrm{T}_{\text {agg }} 75$ ) was determined as $40.8^{\circ} \mathrm{C}$ and $45.5^{\circ} \mathrm{C}$, respectively.

\section{Treatment, $\mu \mathrm{M}$}

\begin{tabular}{llllllll}
\hline 10 & 3.3 & 1.1 & 0.37 & 0.12 & 0.04 & 0.014 & DMSO
\end{tabular}

\section{IB: PPAR-alpha}

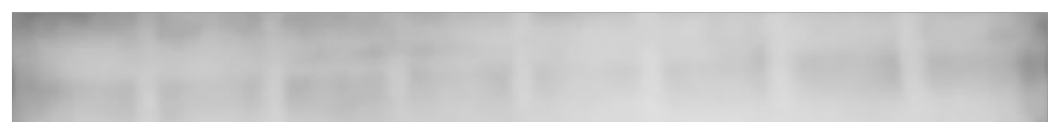

FA

IB: PPAR-alpha

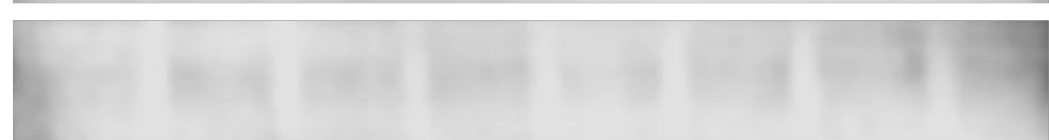

A91

IB: PPAR-alpha

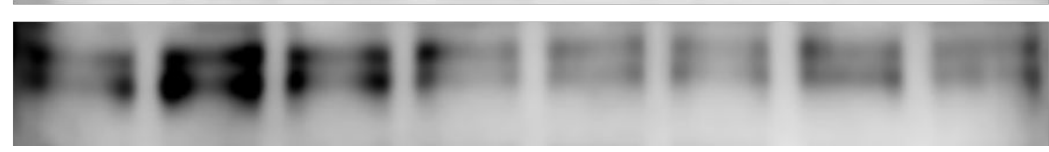

A190

Figure S4. Dose-response CETSA at $45.5^{\circ} \mathrm{C}$. MIO-M1 cells treated with increasing doses of inhibitors were subjected to heat challenge at $\mathrm{T}_{\text {agg }} 75$ of $45.5^{\circ} \mathrm{C}$. Band intensity of remaining stabilized protein at each dose was quantified, and subsequently the $\mathrm{EC}_{50}$ for each inhibitor was determined. 


\section{$\underline{\text { LDH Cytotoxicity Data }}$}

$661 \mathrm{~W}$ mouse photoreceptor cells were cultured on 96-well plate (6700 cells per well) with varying concentrations of DMSO, GW7647, or A91 (0 to $200 \mu \mathrm{M})$ to induce cytotoxicity. Following overnight incubation with the compounds, culture media from the 96-well plate was collected for the LDH quantification assay, which performed following the manufacturer's instructions (Thermo Scientific, \#88953). In short, $50 \mu \mathrm{L}$ of culture medium was mixed and incubated with $50 \mu \mathrm{L}$ of Reaction Mixture at $25{ }^{\circ} \mathrm{C}$ for 30 minutes. $50 \mu \mathrm{L}$ of Stop Solution was added and the absorbance was measured at $490 \mathrm{~nm}$ and $680 \mathrm{~nm}$. Cell death (percentage of maximum LDH release control) was then determined following the manufacturer's protocols.

\section{LDH Cytotoxicity Assay (661W cell)}

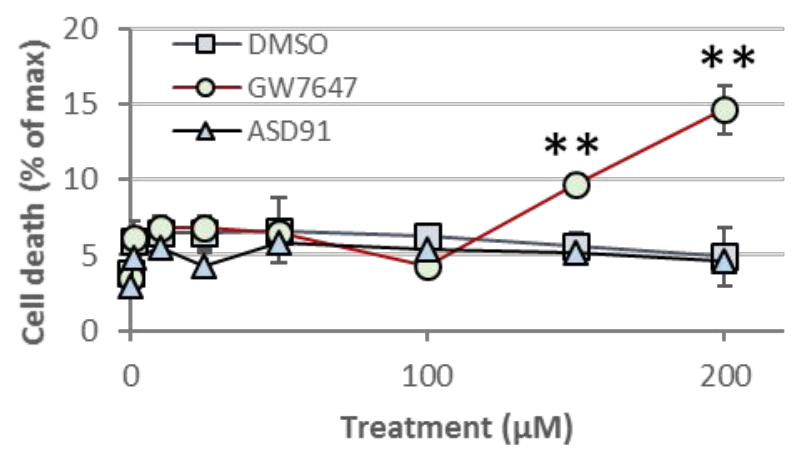

Figure S5. Cytotoxicity of A91 in a retinal epithelium photoreceptor cell-line 661W. LDH activities were measured to determine dose-dependent cytotoxicity of ASD091. No significant LDH activities were detected in all concentrations of ASD091 tested, whereas GW7647 began to induce cell death $150 \mu \mathrm{M}$ (mean $\pm \mathrm{SD} ; n=3)$. $* * P<0.01$.

\section{DMSO}
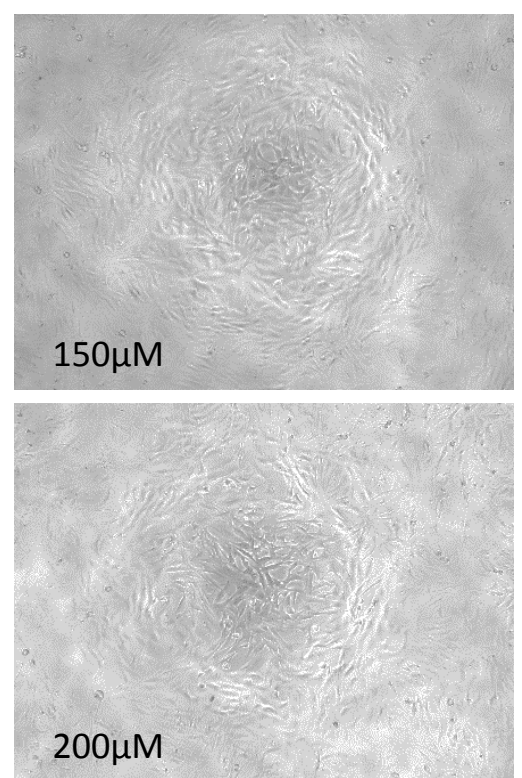

GW7647

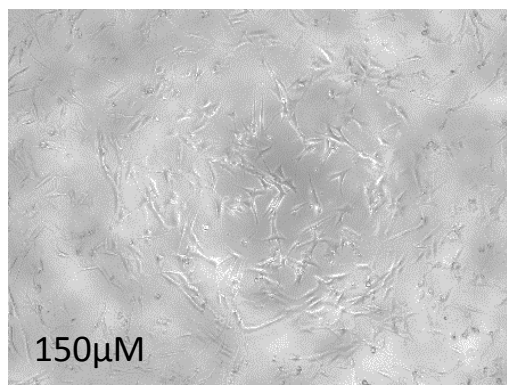

$200 \mu \mathrm{M}$

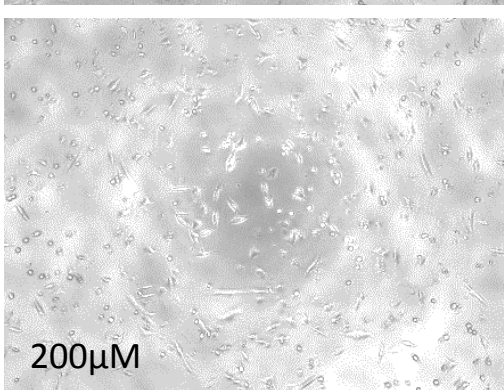

ASD091

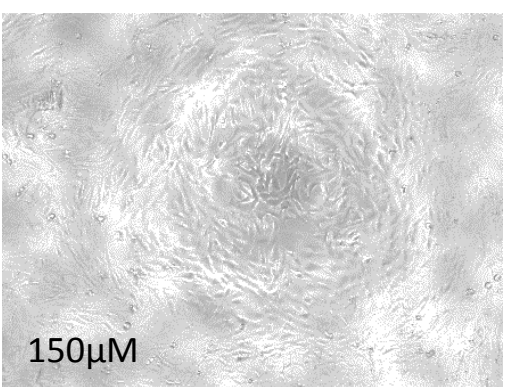

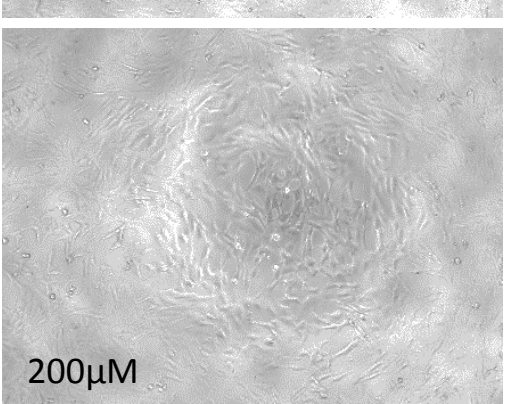

Figure S6. Cellular morphology after compound treatment under light microscopy. Unlike GW7647, ASD091-treated cells did not exhibit any obvious signs of cellular morphology changes at all concentrations tested. 


\section{$\underline{\text { In silico Metabolic Predictions }}$}
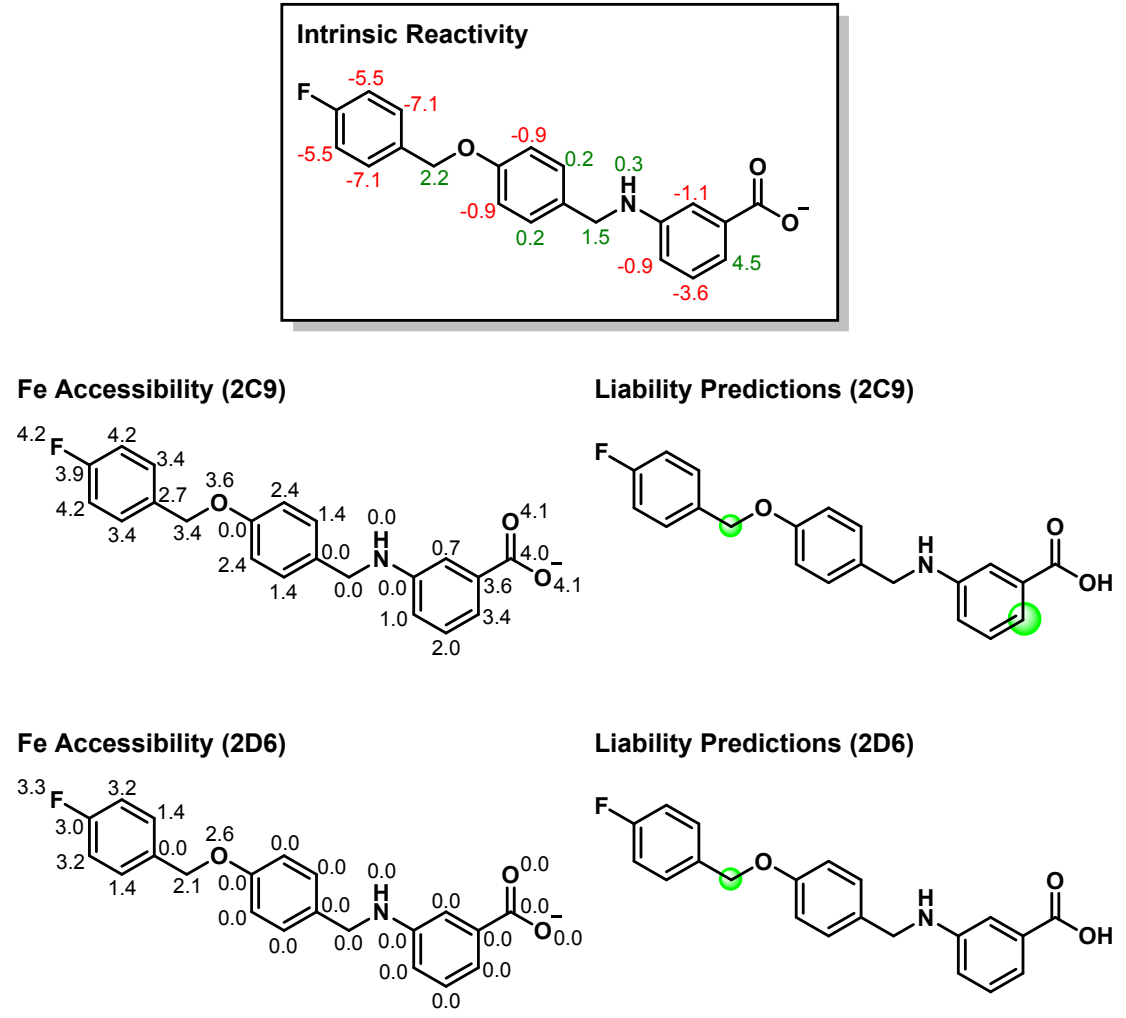

Liability Predictions (2D6)<smiles>O=C(O)c1cccc(NCc2ccc(O[Hg]c3ccc(F)cc3)cc2)c1</smiles>

Liability Predictions (3A)

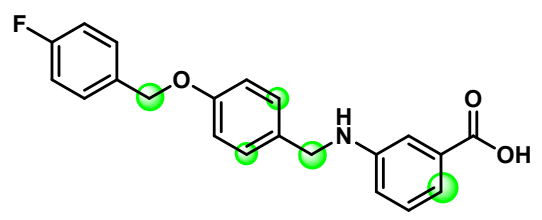

Figure S7. In silico metabolic predictions determined by Schrodinger's P450 Site of Metabolism Module. Predictions calculated for $\mathbf{4 b}$ against 2C9, 2D6, and 3A4 CYPs. Components are described as transcribed directly from the manufacturer's manual. Intrinsic Reactivity $=$ atoms are labeled with the intrinsic reactivity calculated with Hammett and Taft methodology. Positive values are predicted to be more reactive, negative values are predicted to be less reactive. Fe Accessibility = atoms are labeled with accessibility to the iron. This is defined as the natural logarithm of the number of poses for the atom in which the atom was within $5 \AA$ of Fe. Larger values indicate greater accessibility. Liability Predictions = linear combination of the accessibility and the intrinsic reactivity. Results are displayed as green circles, in which the radius is proportional to the score. Larger circles mean higher reactivity. 
FINAL COMPOUND ${ }^{1} \mathrm{H}$ and ${ }^{13} \mathrm{C}$ NMR SPECTRA

$4 \mathrm{~b}$

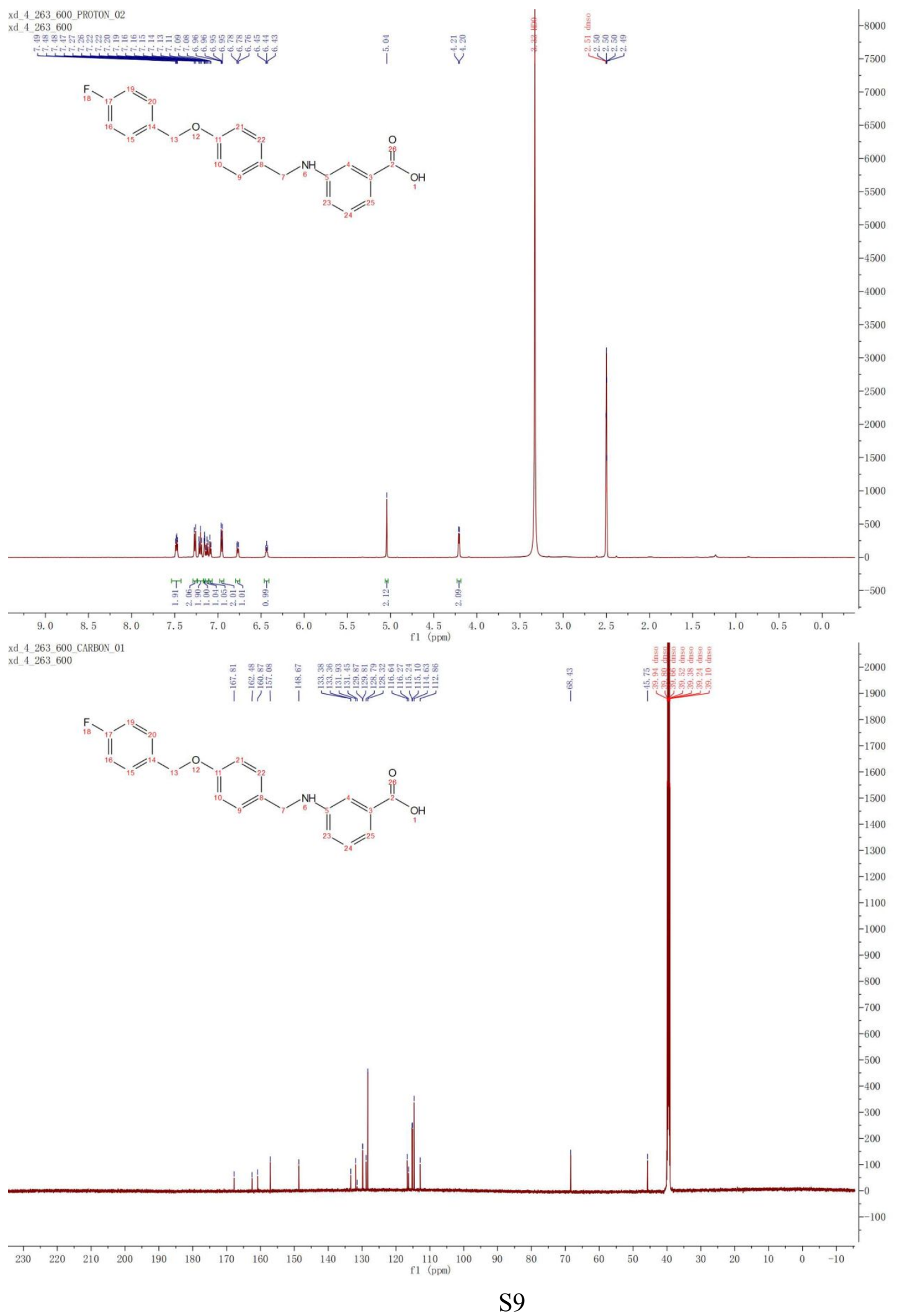




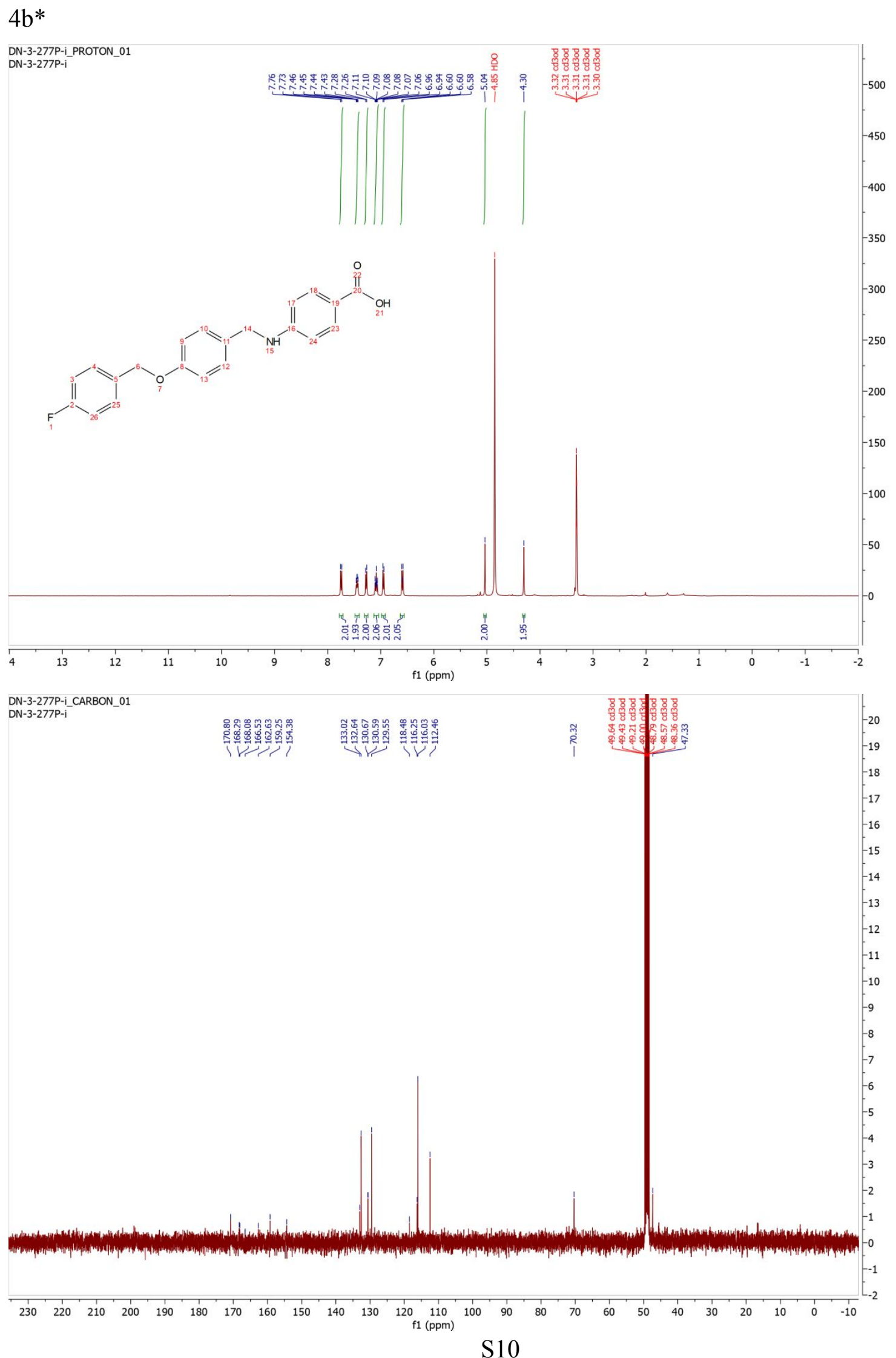


$4 c$

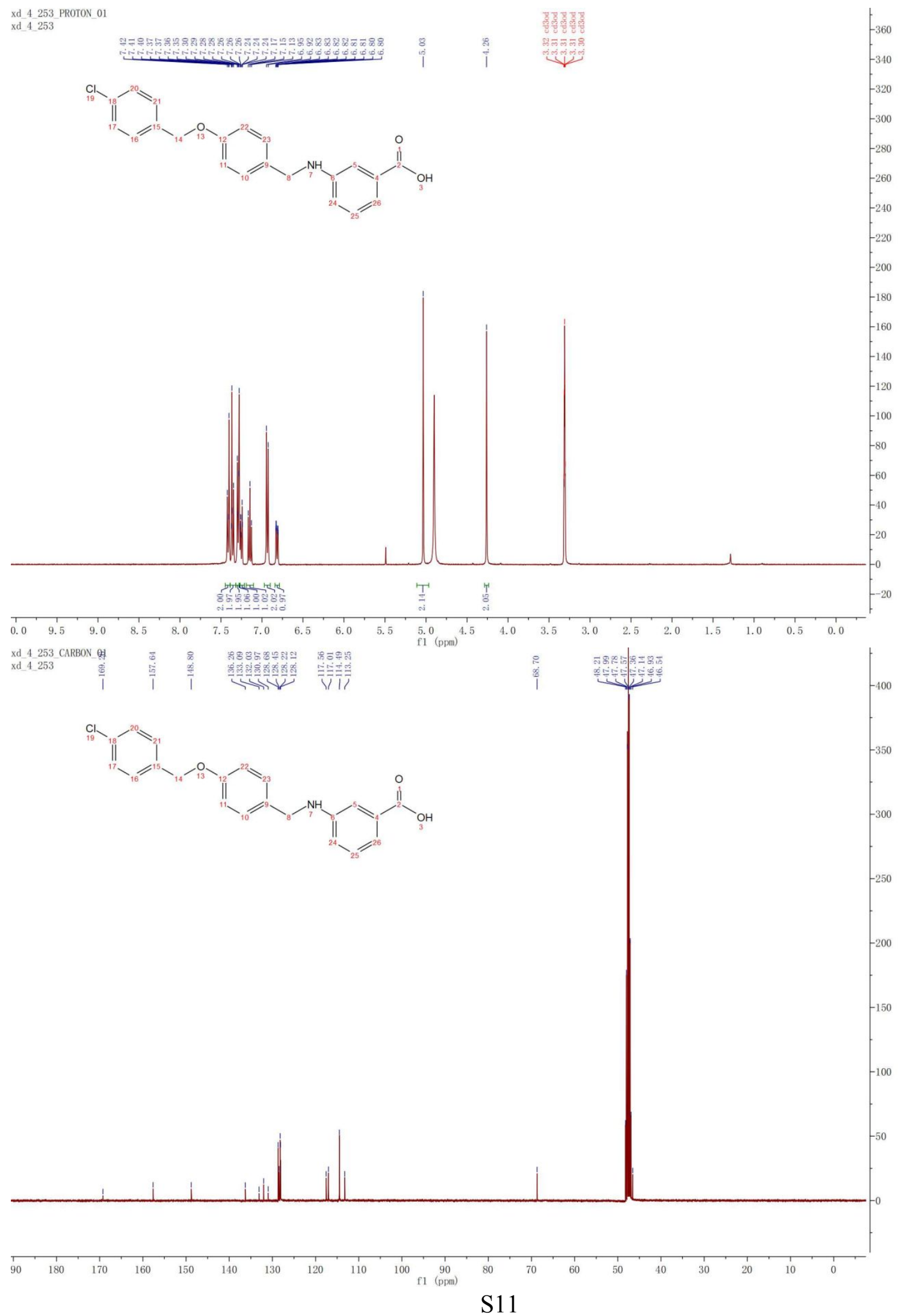


$4 d$

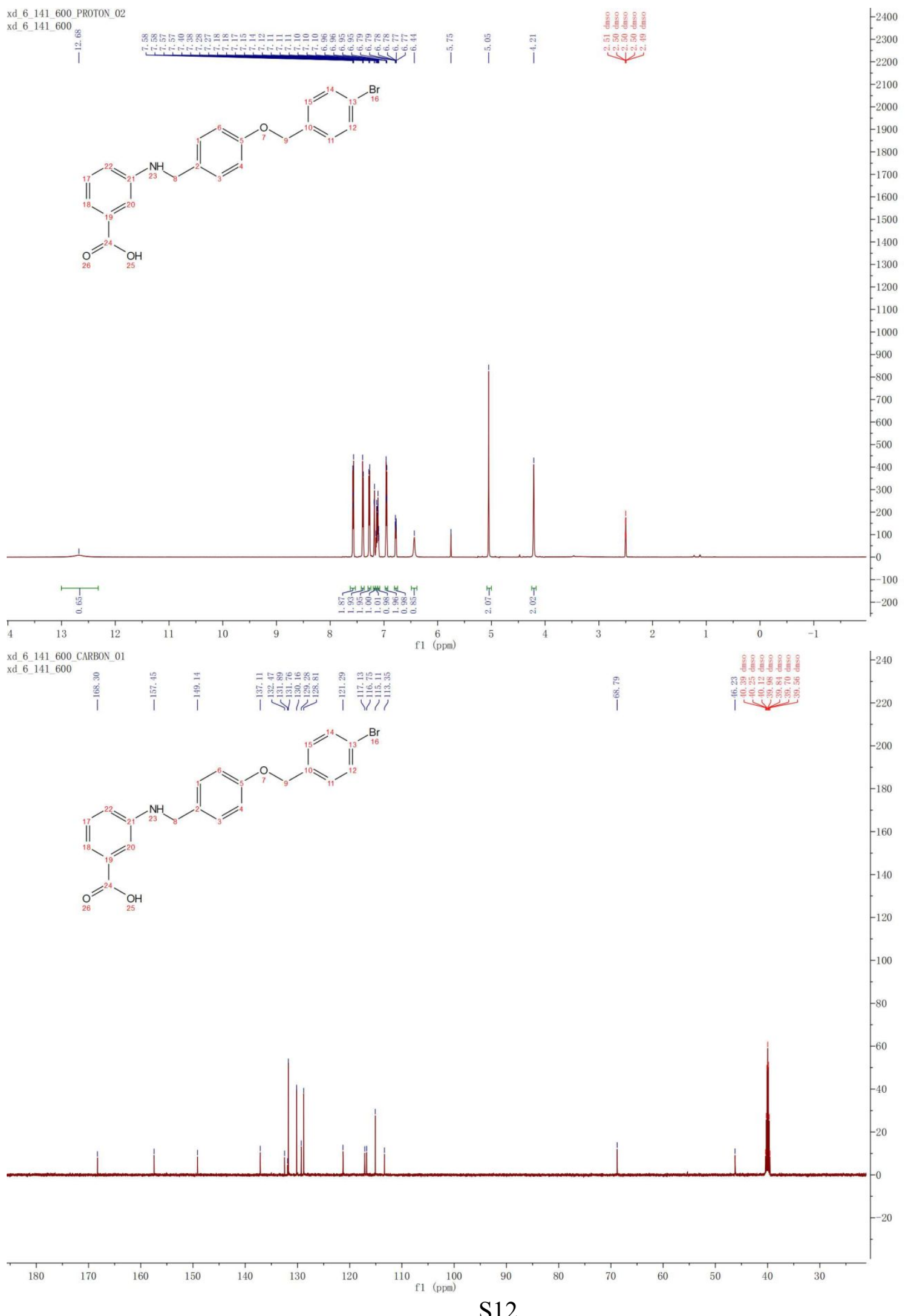


$4 e$

xd 6 $6127 \quad 600$ PROTON 02

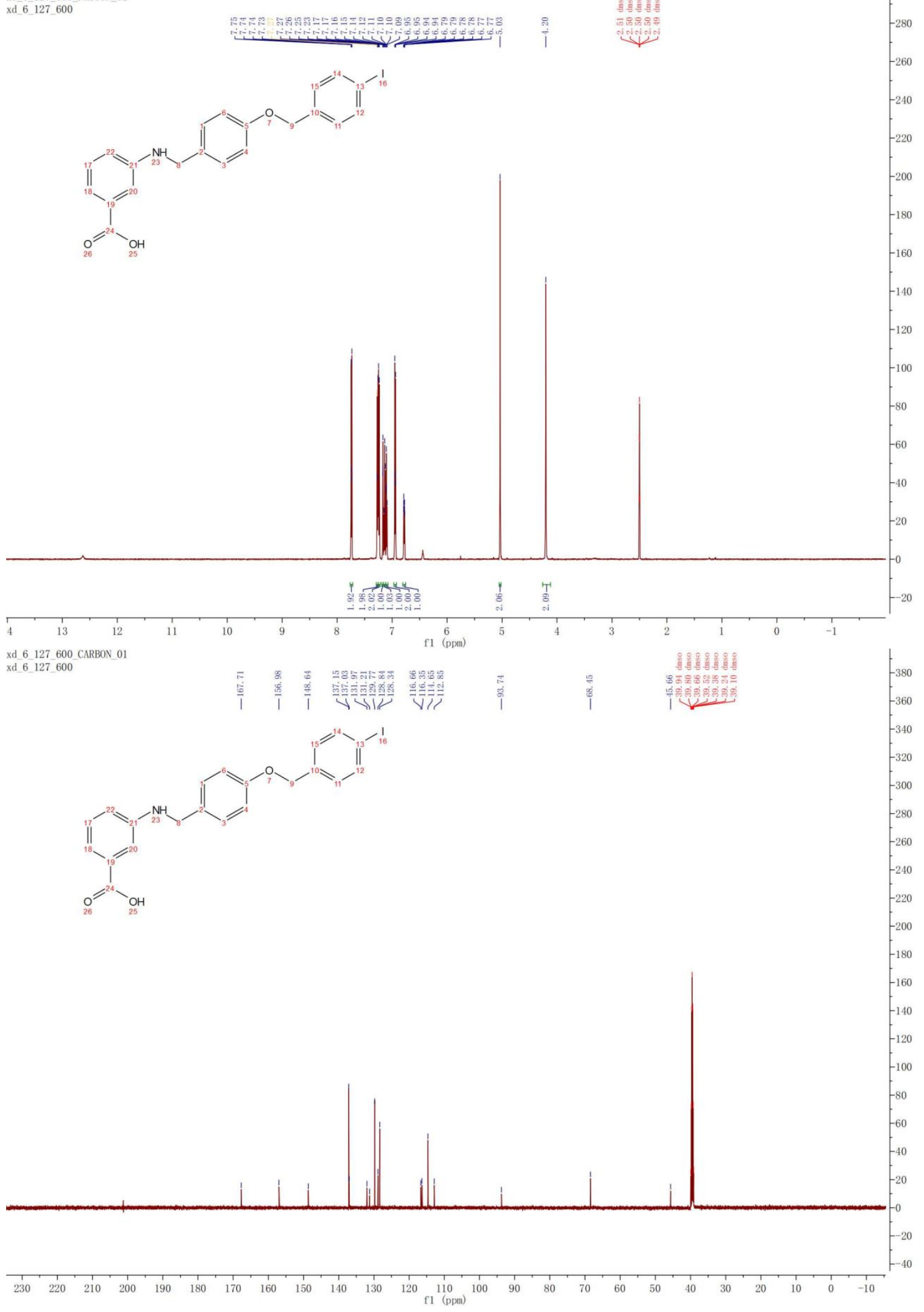

S13 


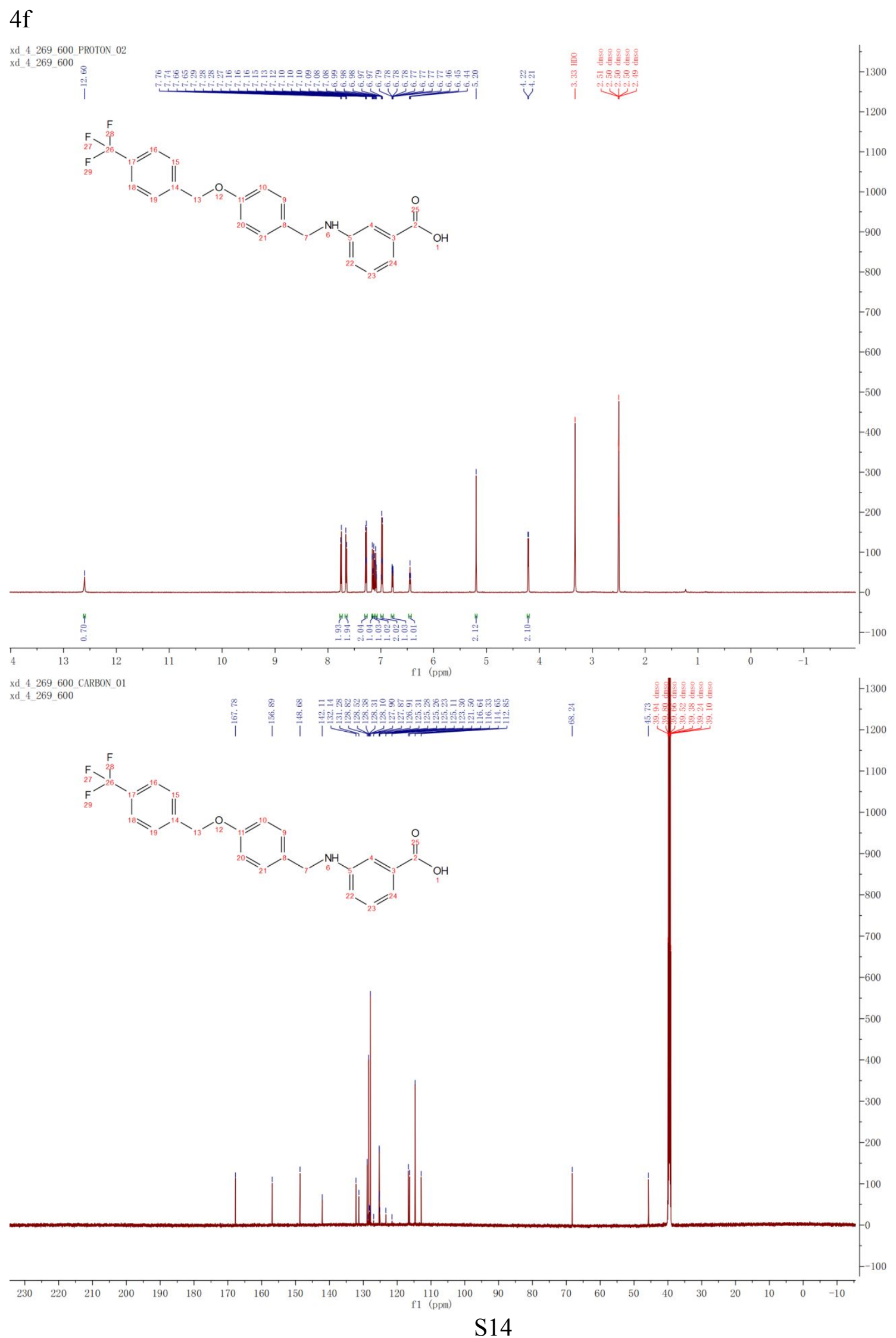




\section{$4 \mathrm{~g}$}

xd 6 6131 600 PROTON 02

xd $6 \quad 131 \quad 600$

।
ป
ป

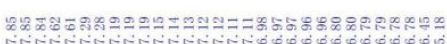

${ }^{2}$

11:198

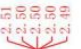

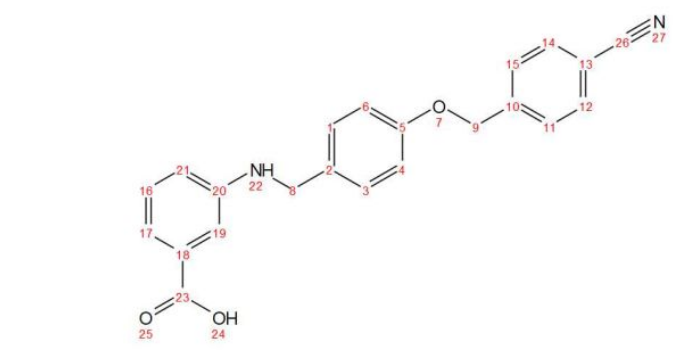

$-2500$
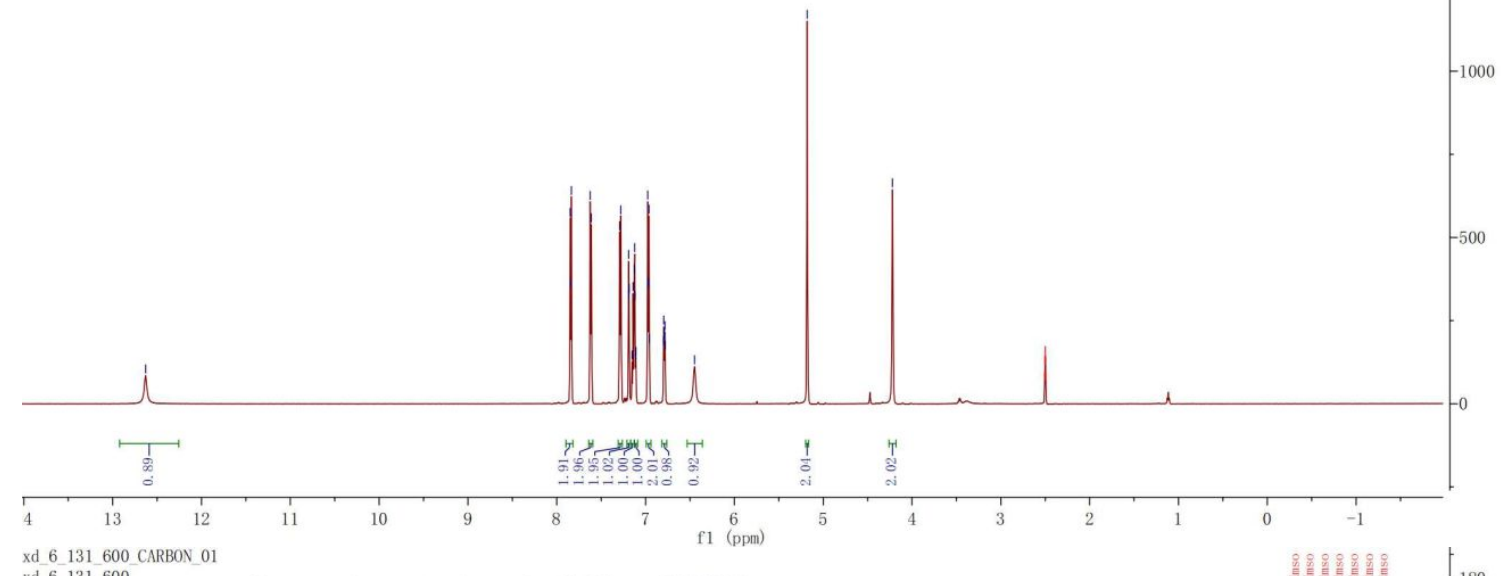

xd 6 6 131_600 CARBON_01

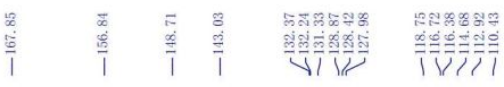<smiles></smiles>

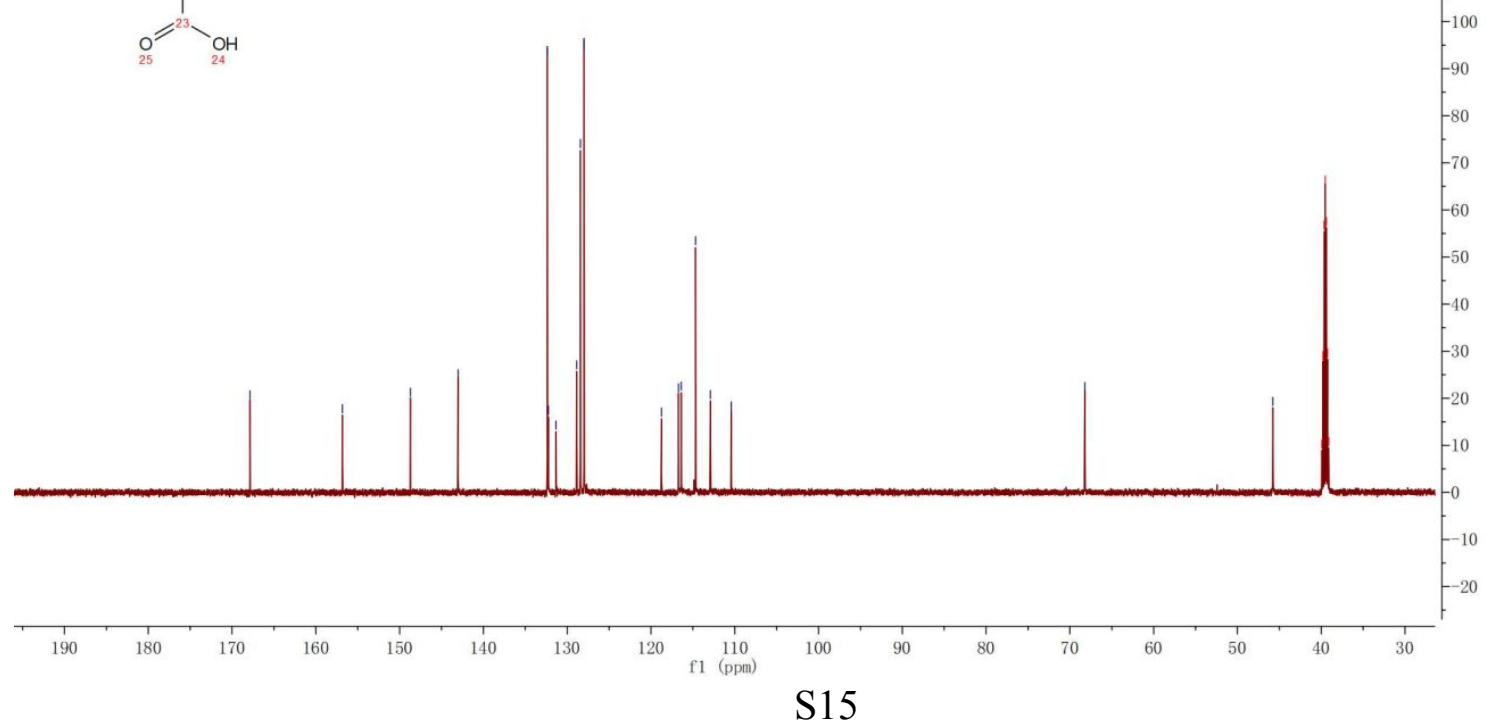


4h

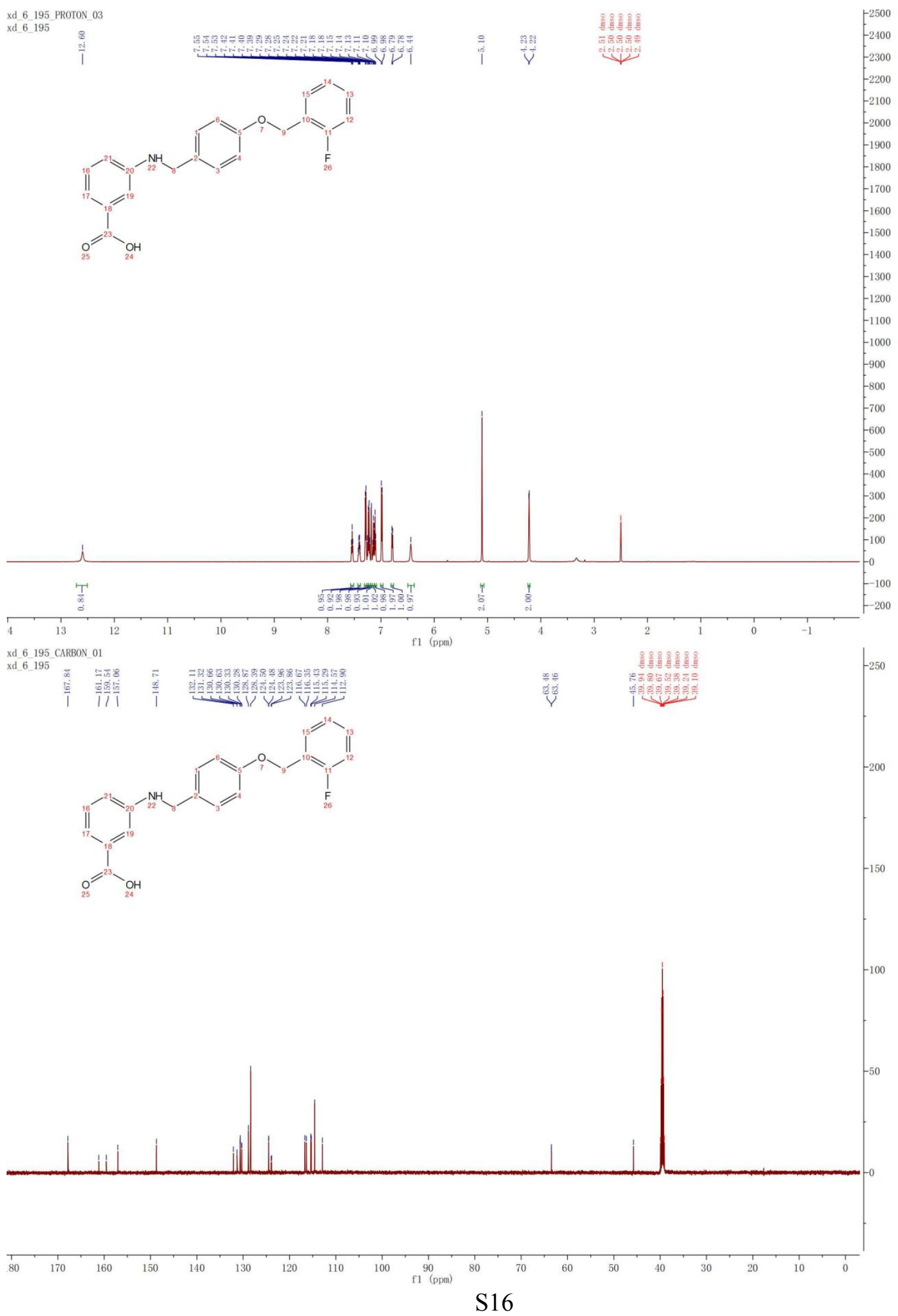




\section{$4 \mathrm{i}$}

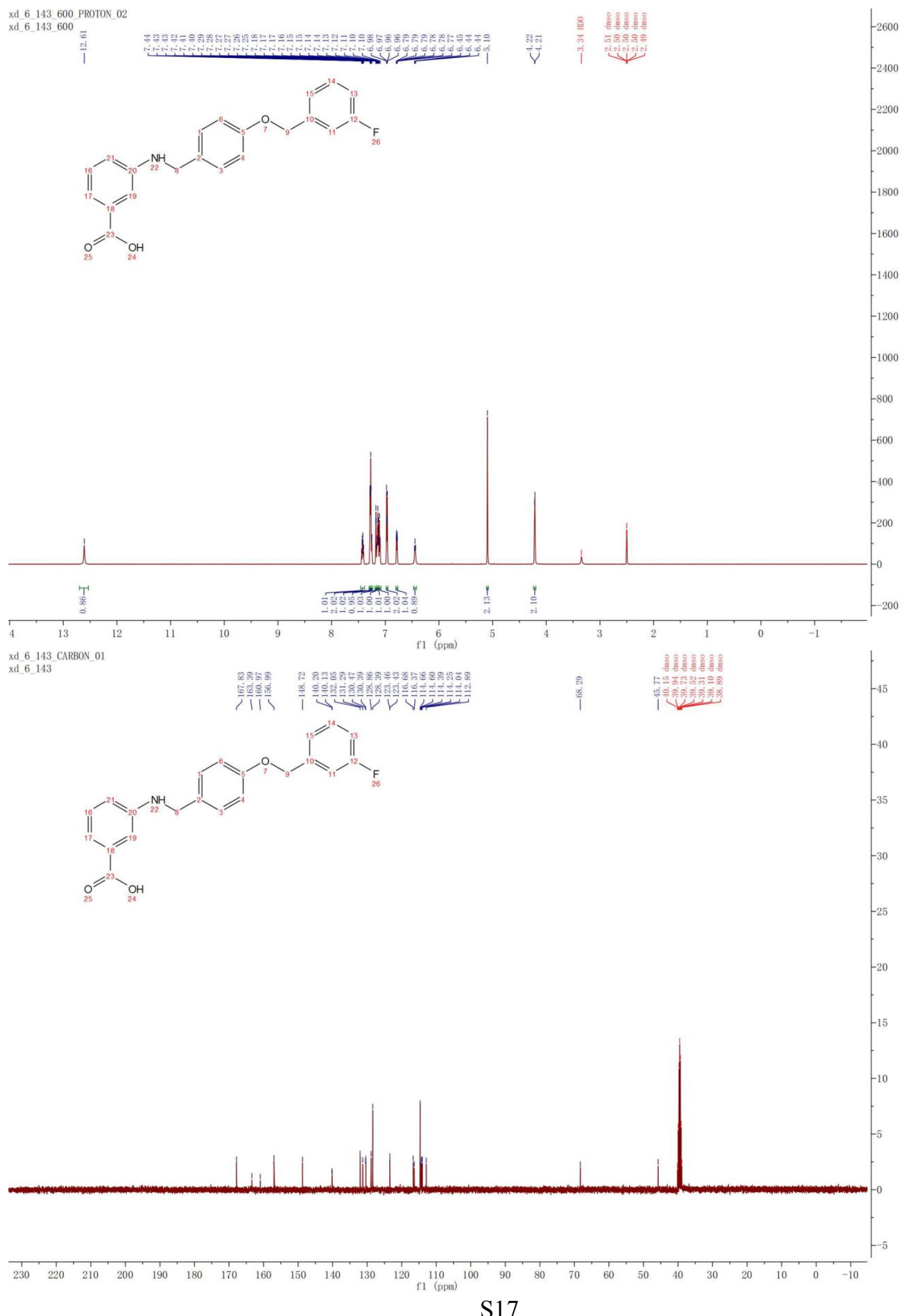

S17 
$4 j$

xd 6145600 PROTON 02

xd 6145600

6 145600

i

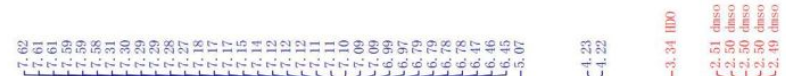

i<smiles></smiles><smiles>CCC</smiles><smiles>CCC</smiles>

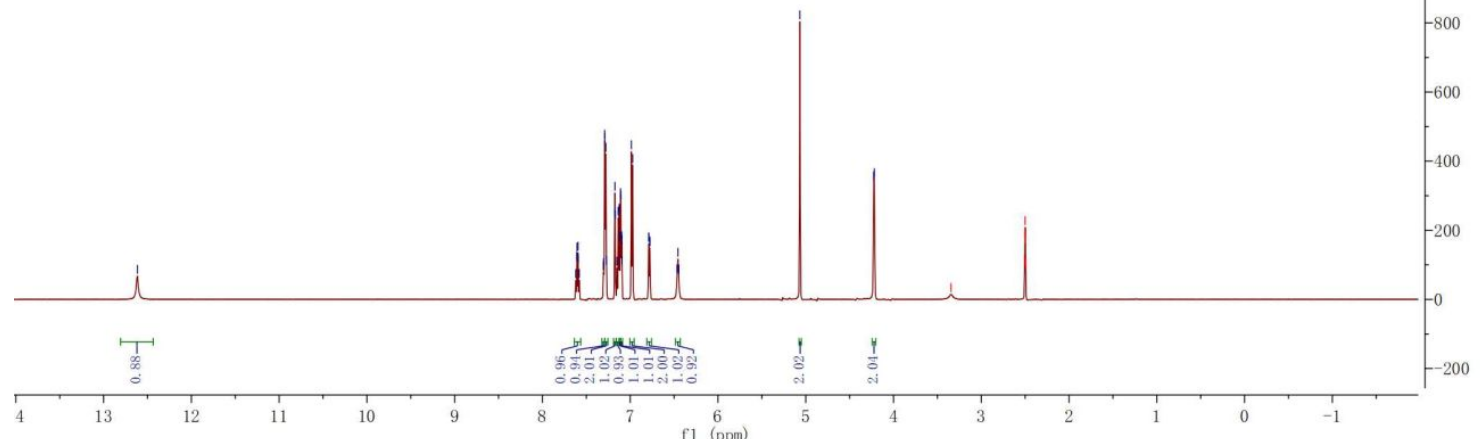

xd 6.145600 CARBON 01 xd $6 \_145600$

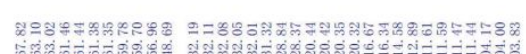

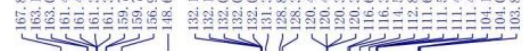

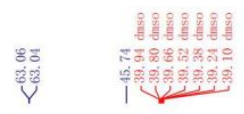<smiles></smiles>

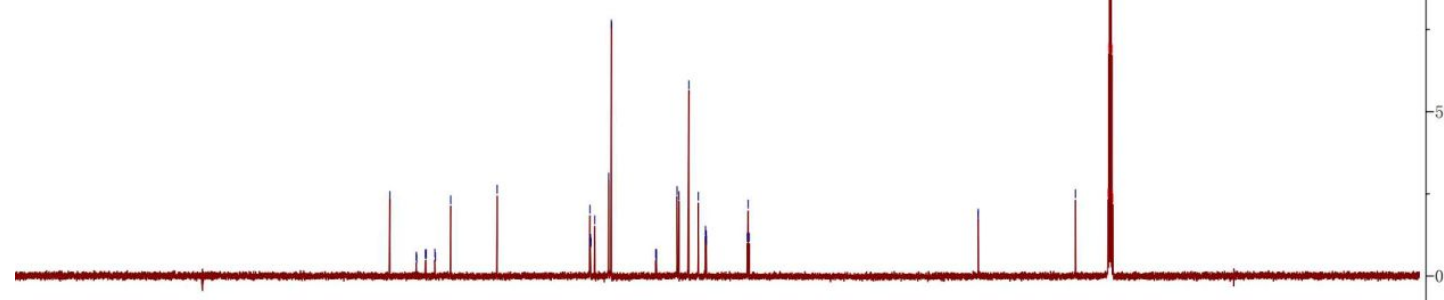$$
23
$$

$230 \quad 2$

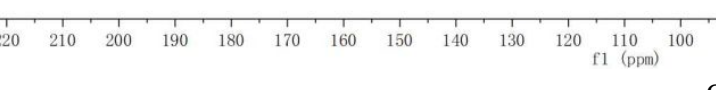

S18 


\section{$4 \mathrm{k}$}
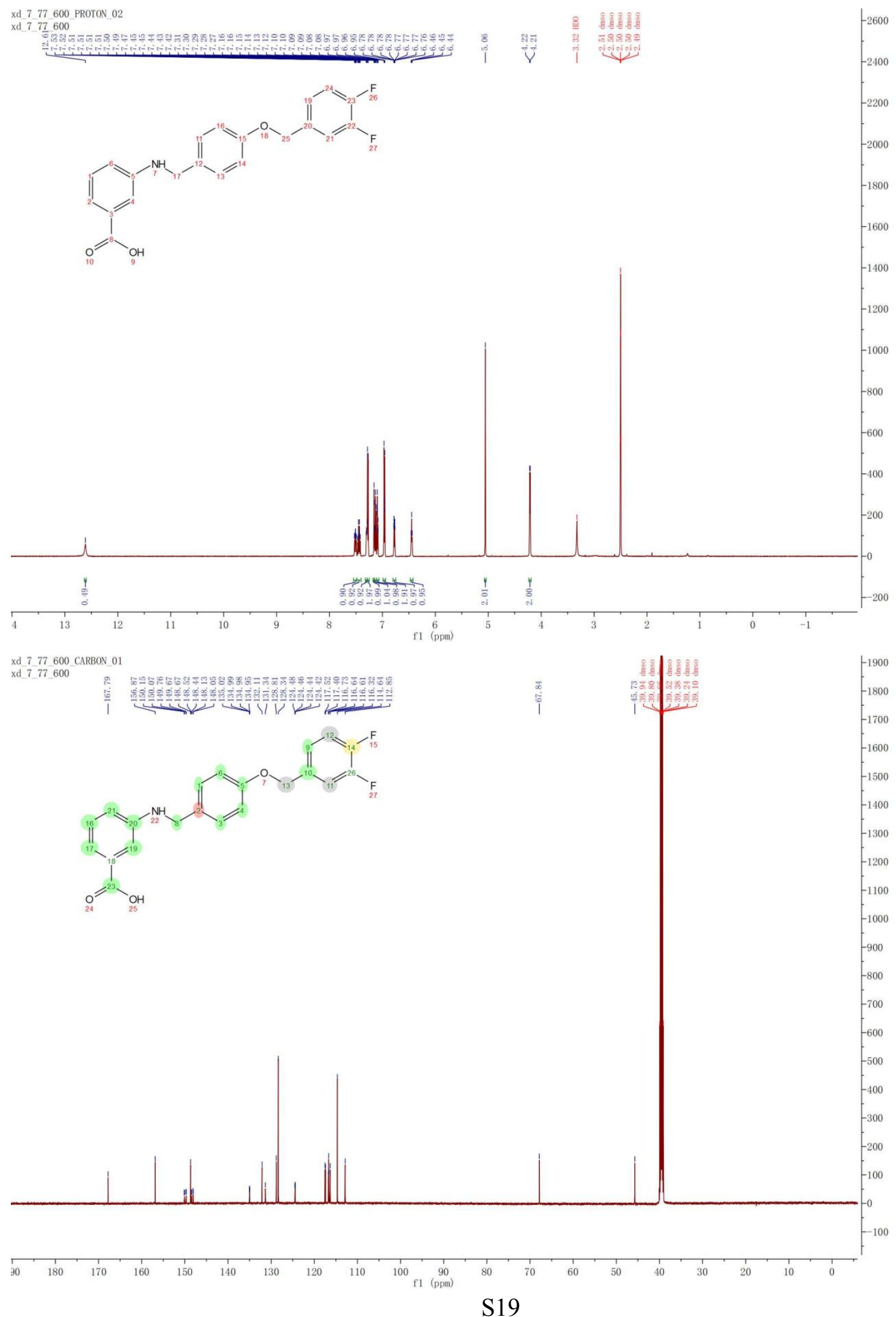
41

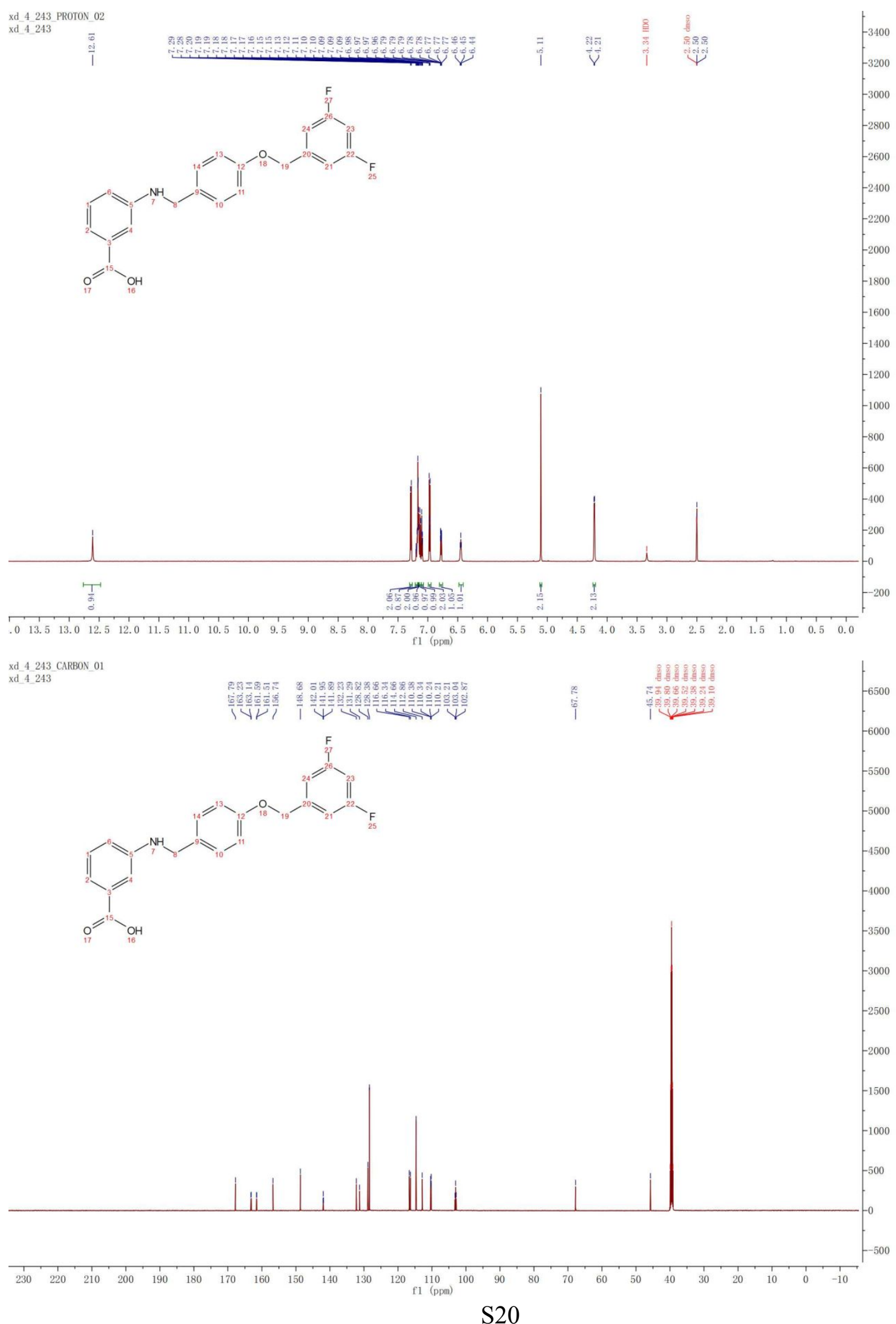




\section{$4 m$}

xd 569 PROTON 01
xd 5 - 69
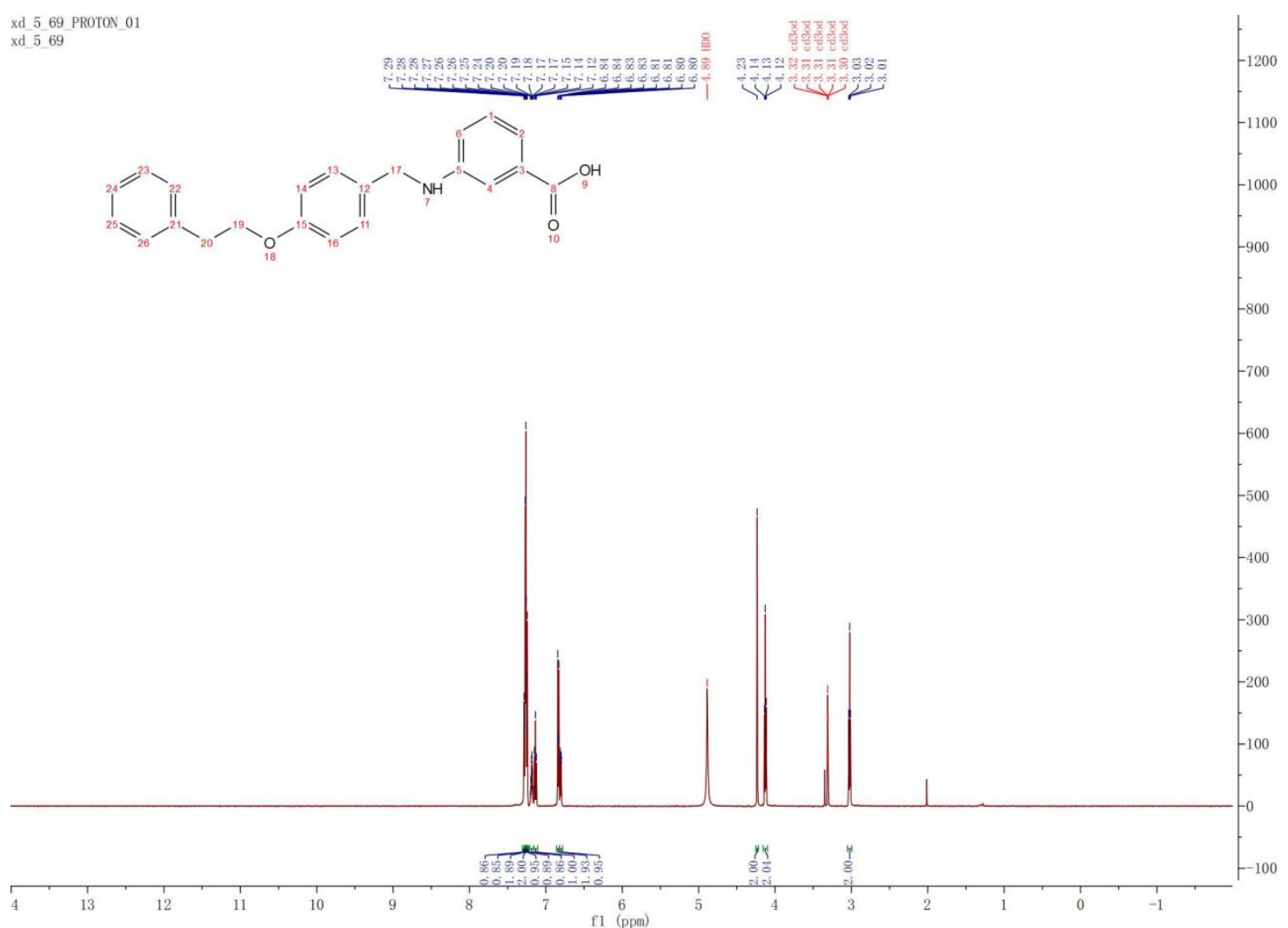

xd 5 69 CARBON 01

xd 56
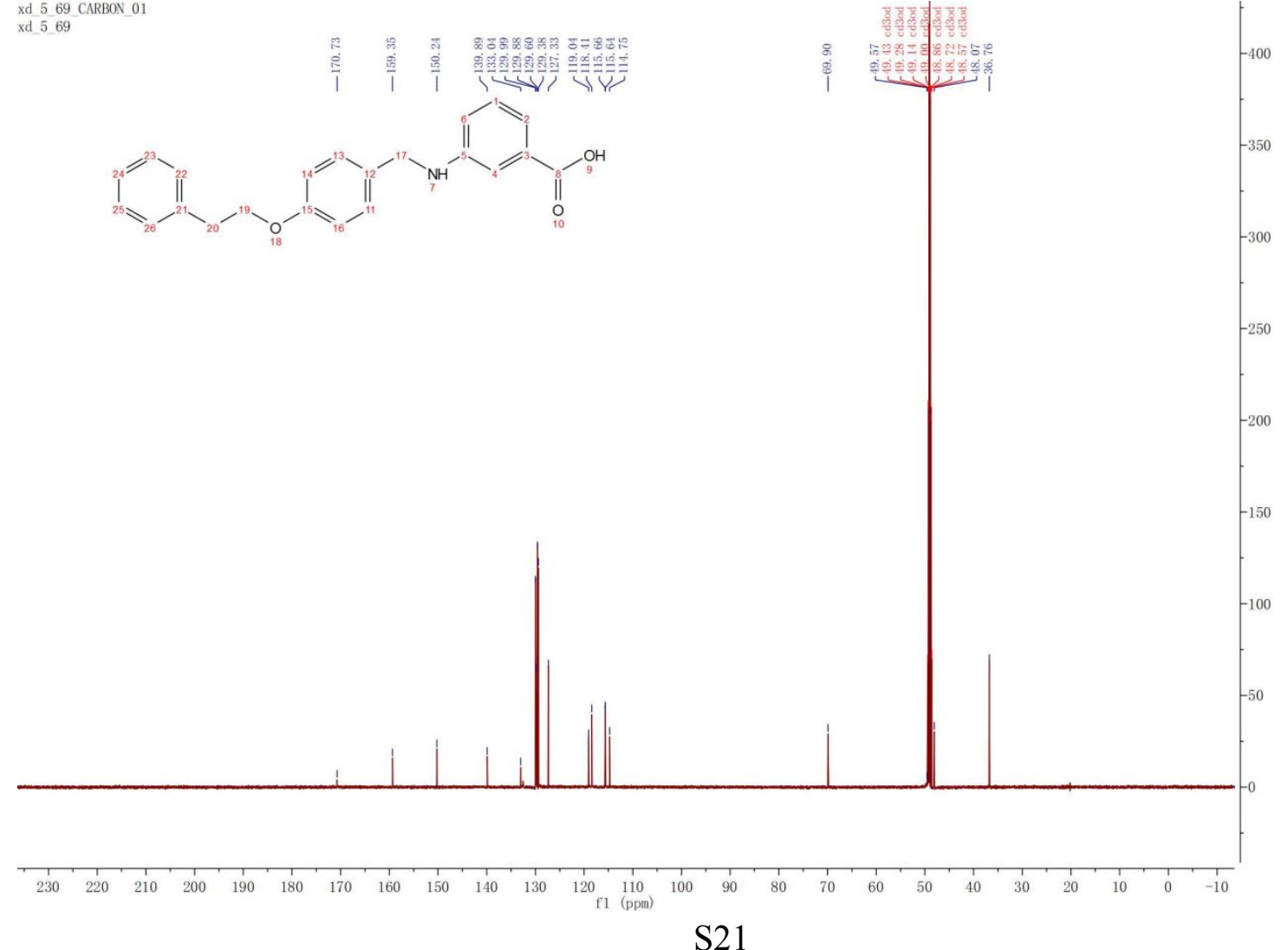
$4 n$

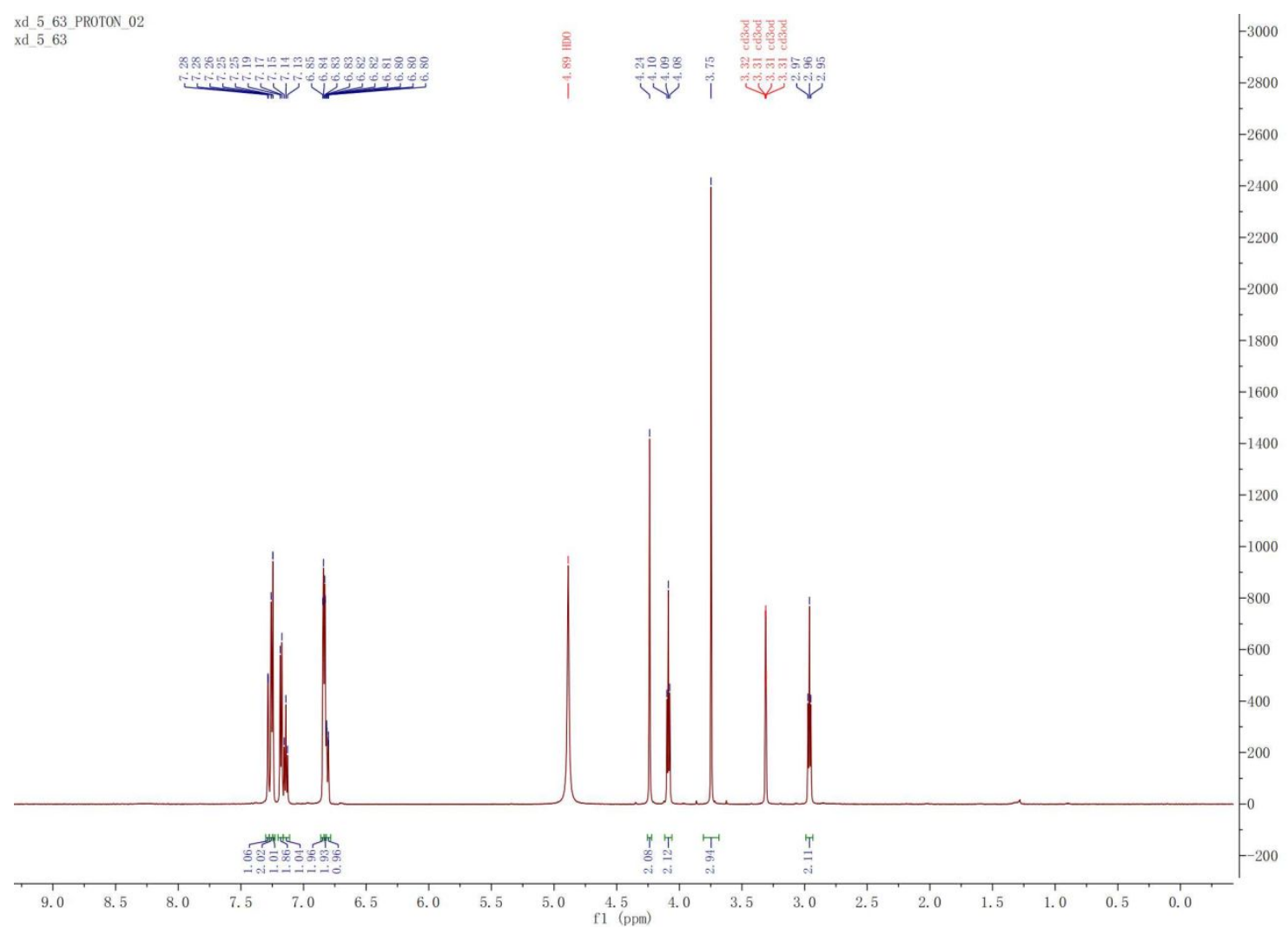

xd 5 63 CARBON 01 xd 563
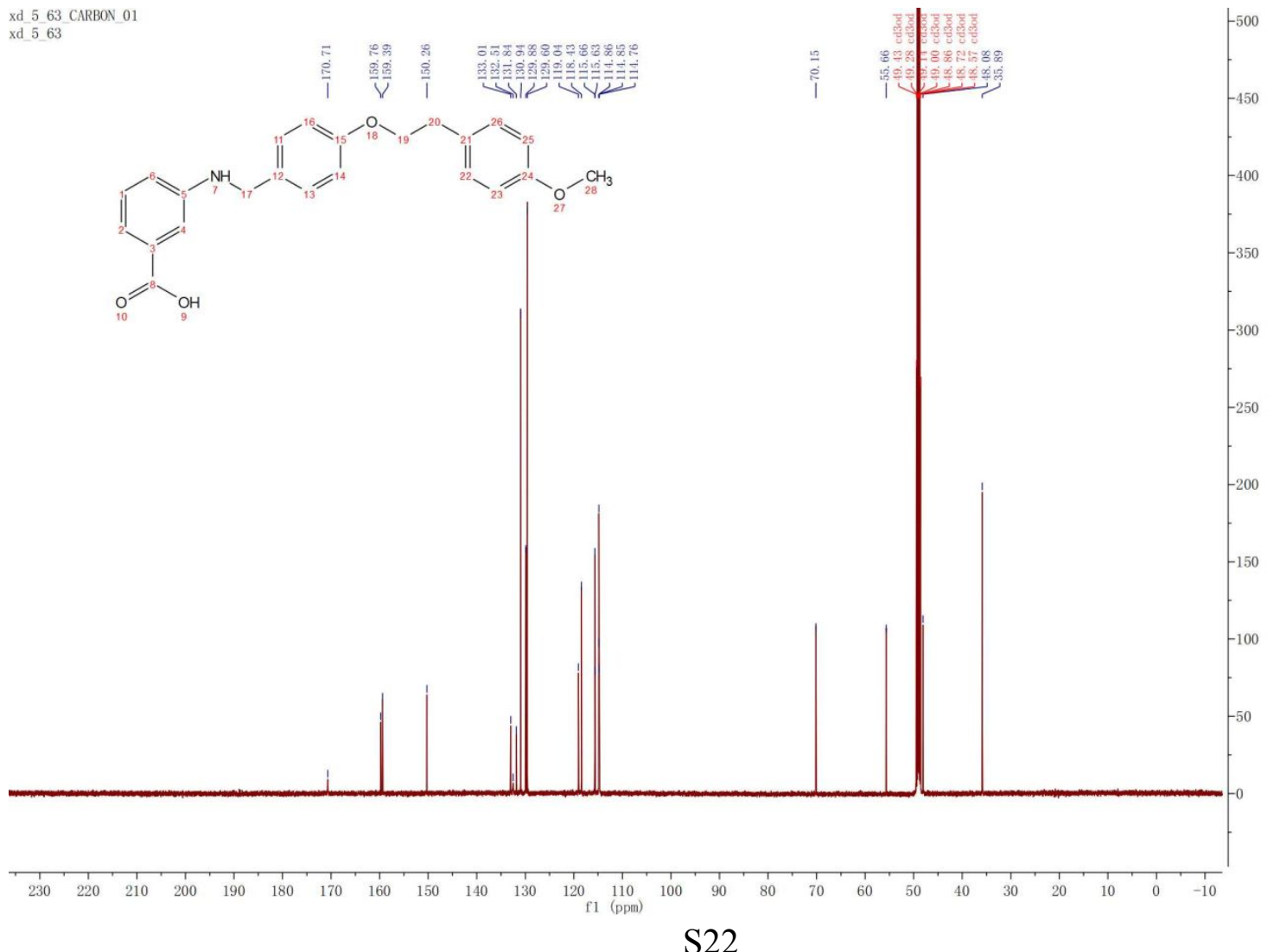
40

xd 5 - 75 PROTON 01
xd 575

$$
01
$$

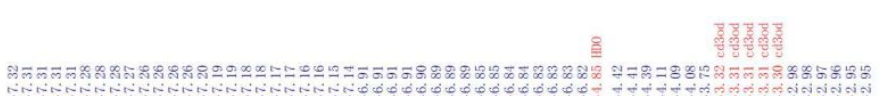

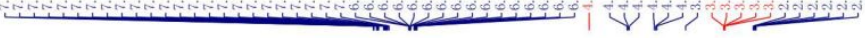

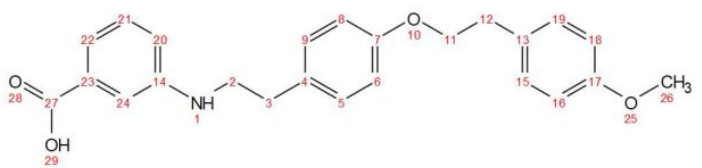

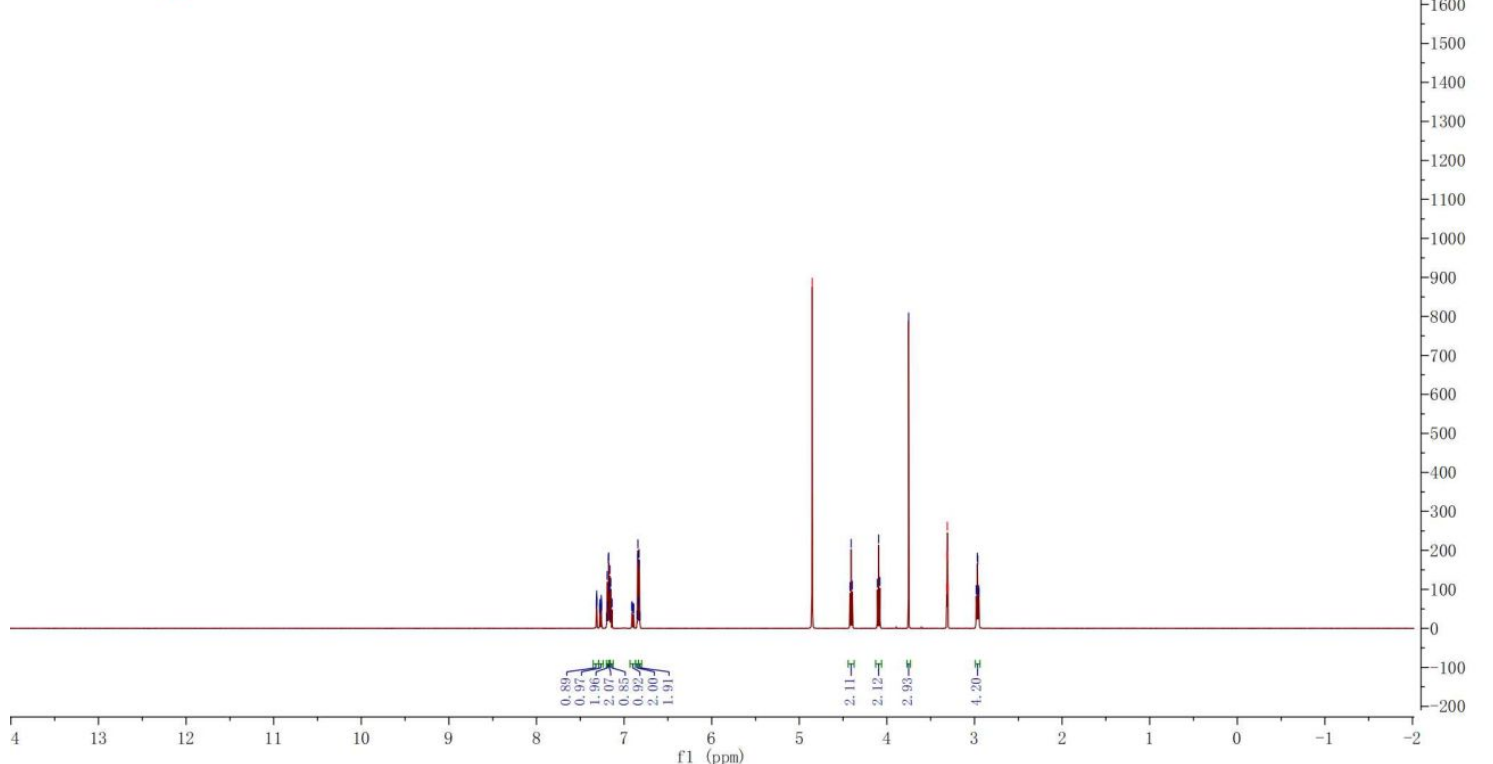
xd 5 . 75 CARBON 01 $\mathrm{xd} 5 \mathbf{5} 75$
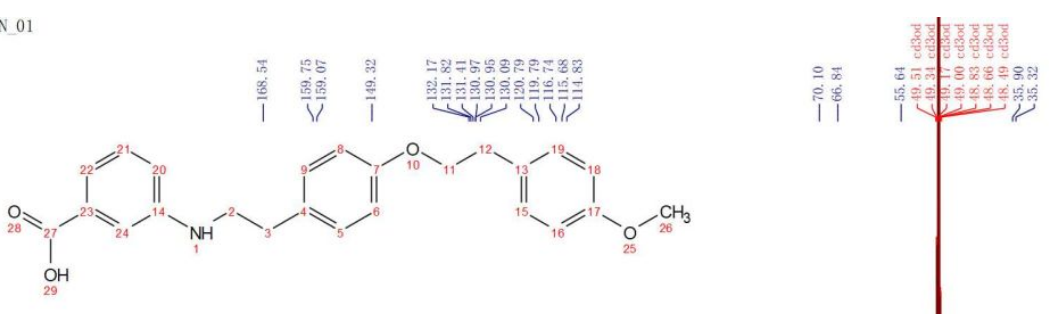

$-130$

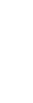




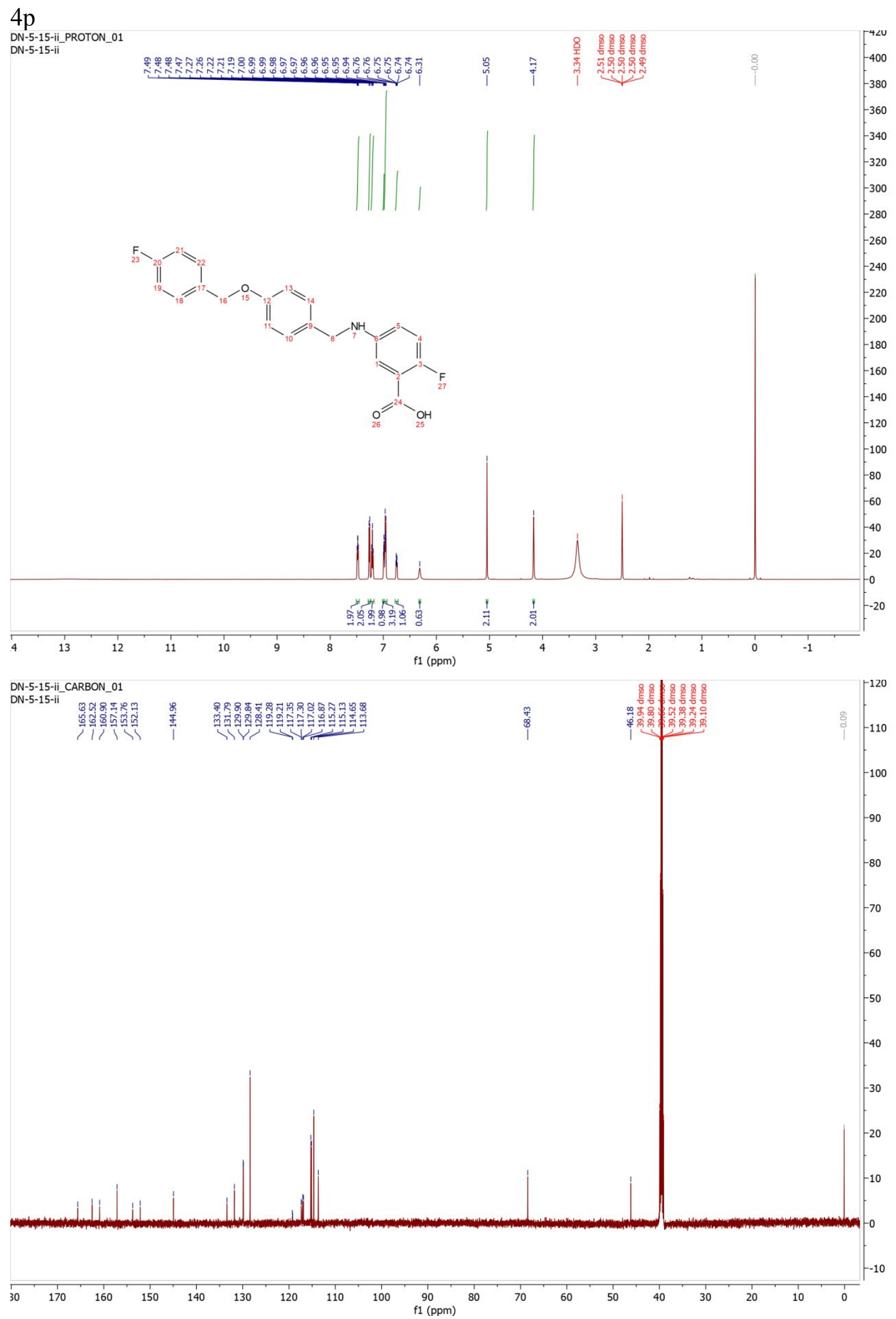

S24 


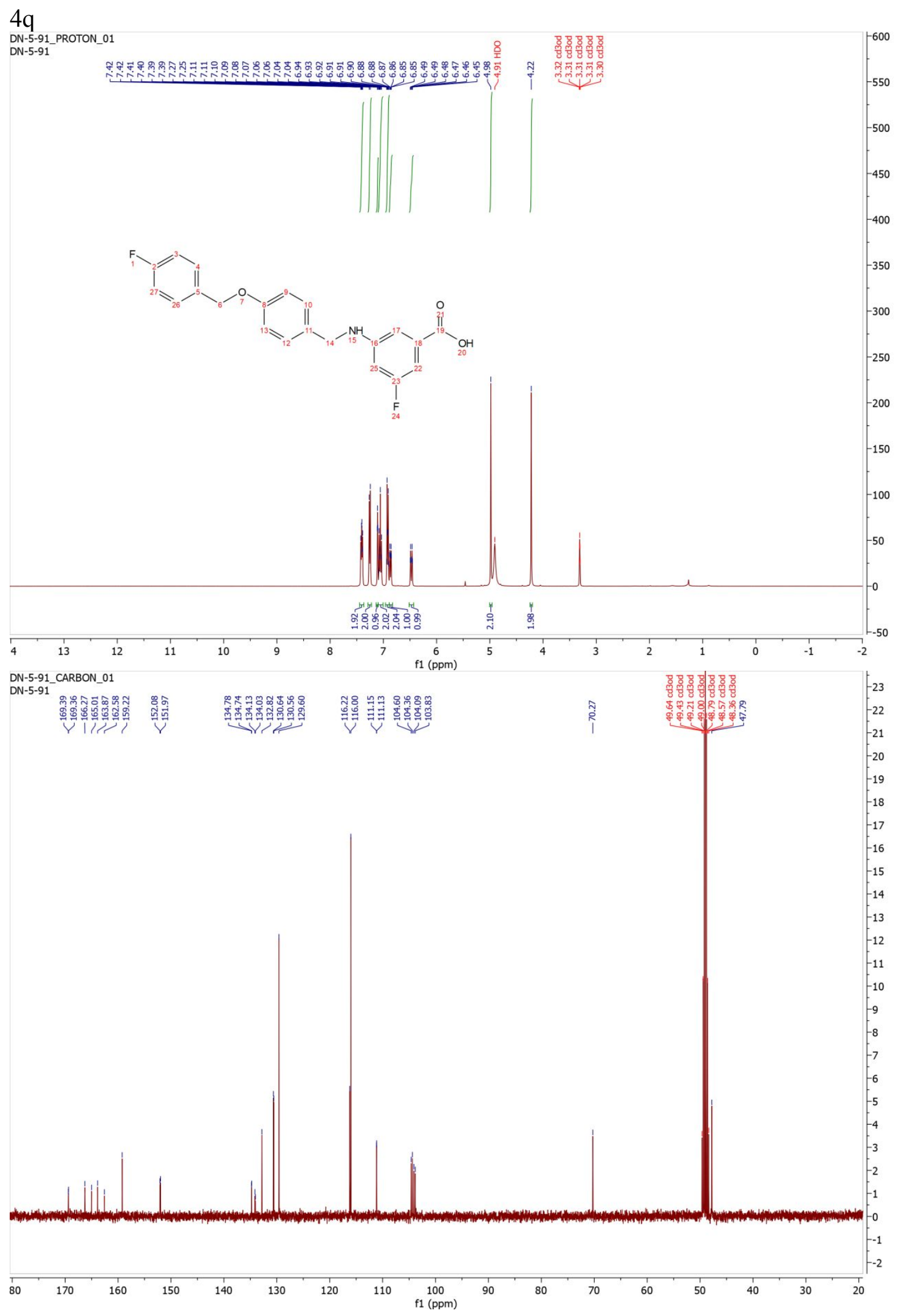


$4 \mathrm{r}$

DN-5-43-i_PROTON_01
DN-5-43-i

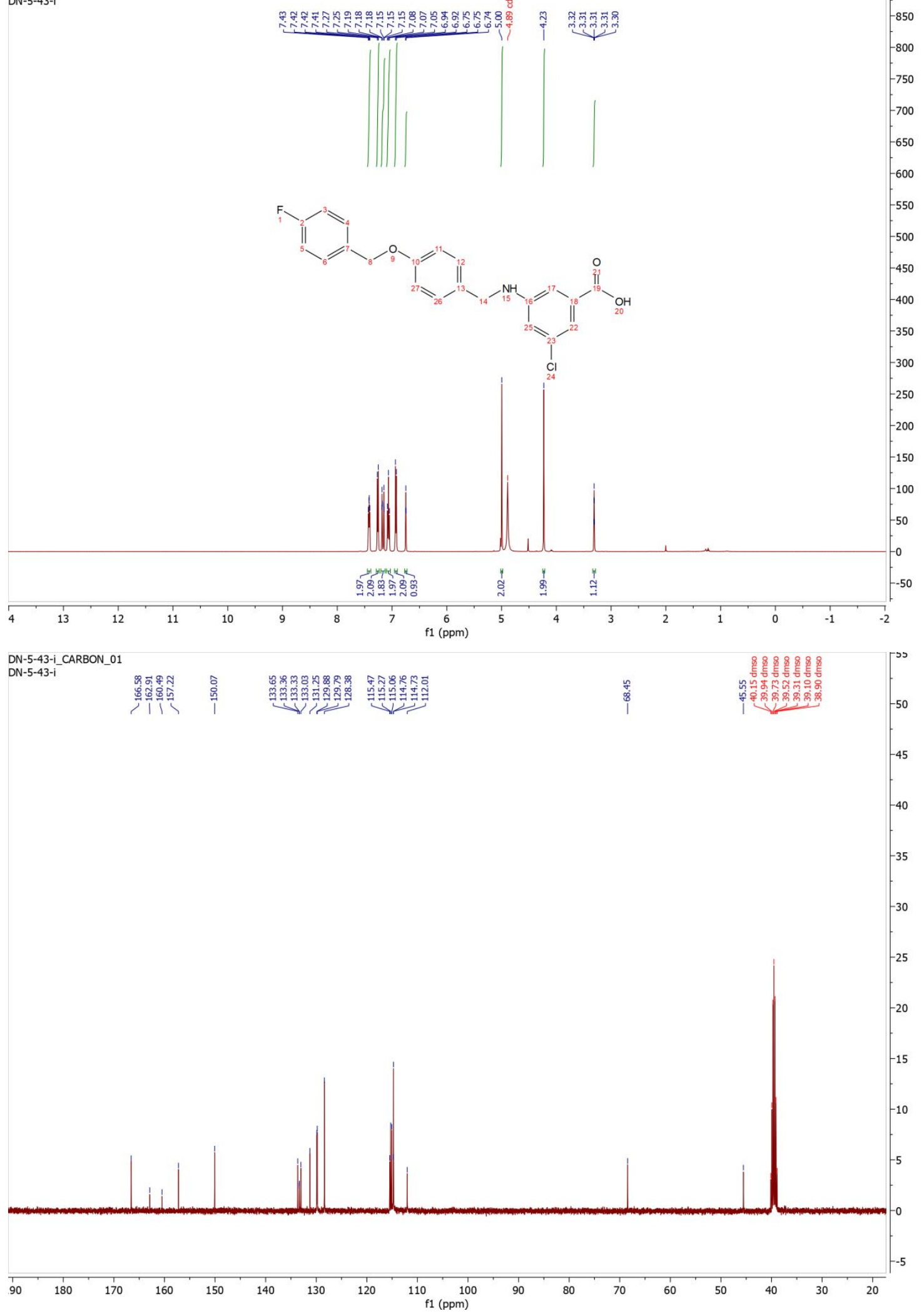

S26 


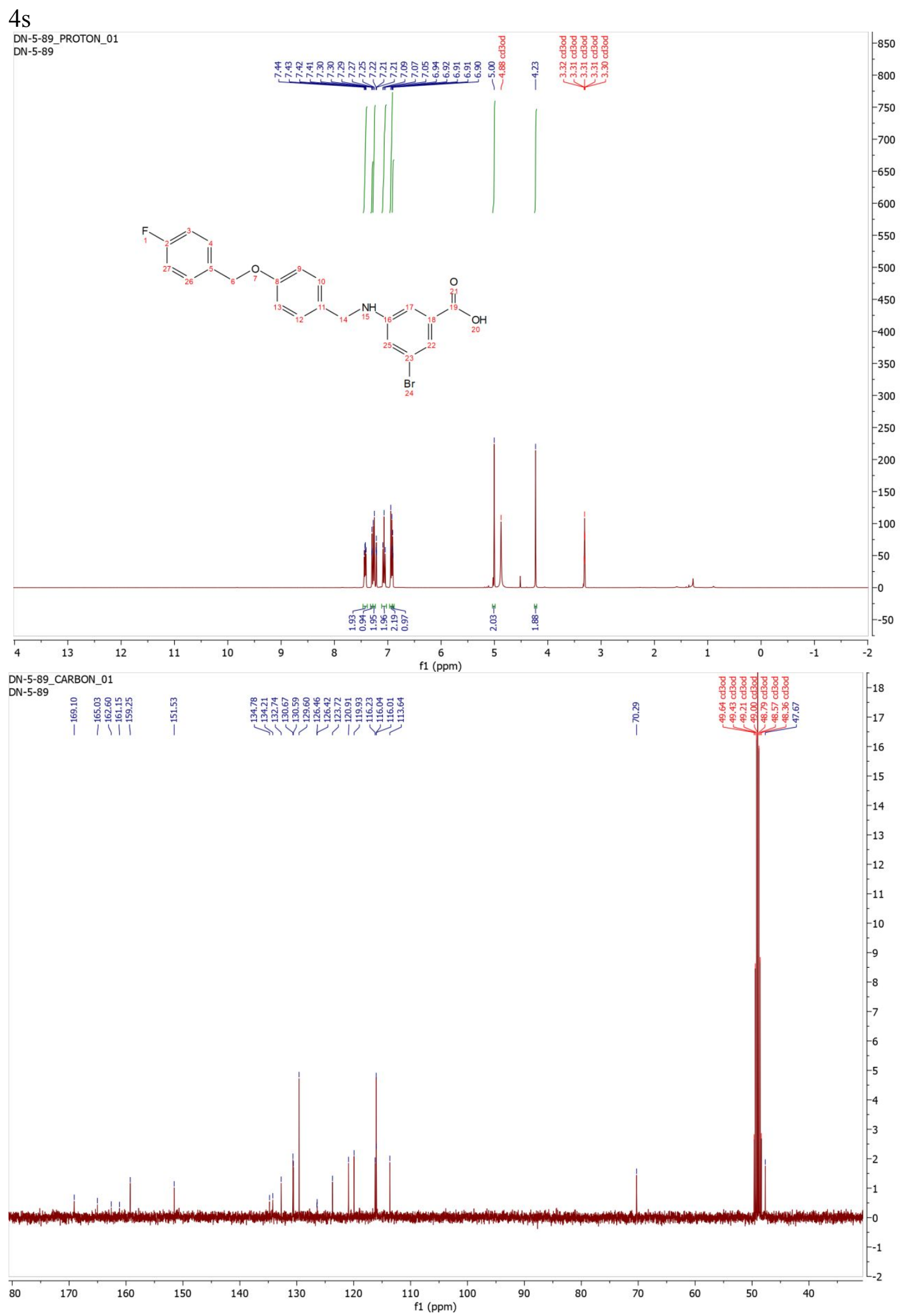

S27 
$4 \mathrm{t}$

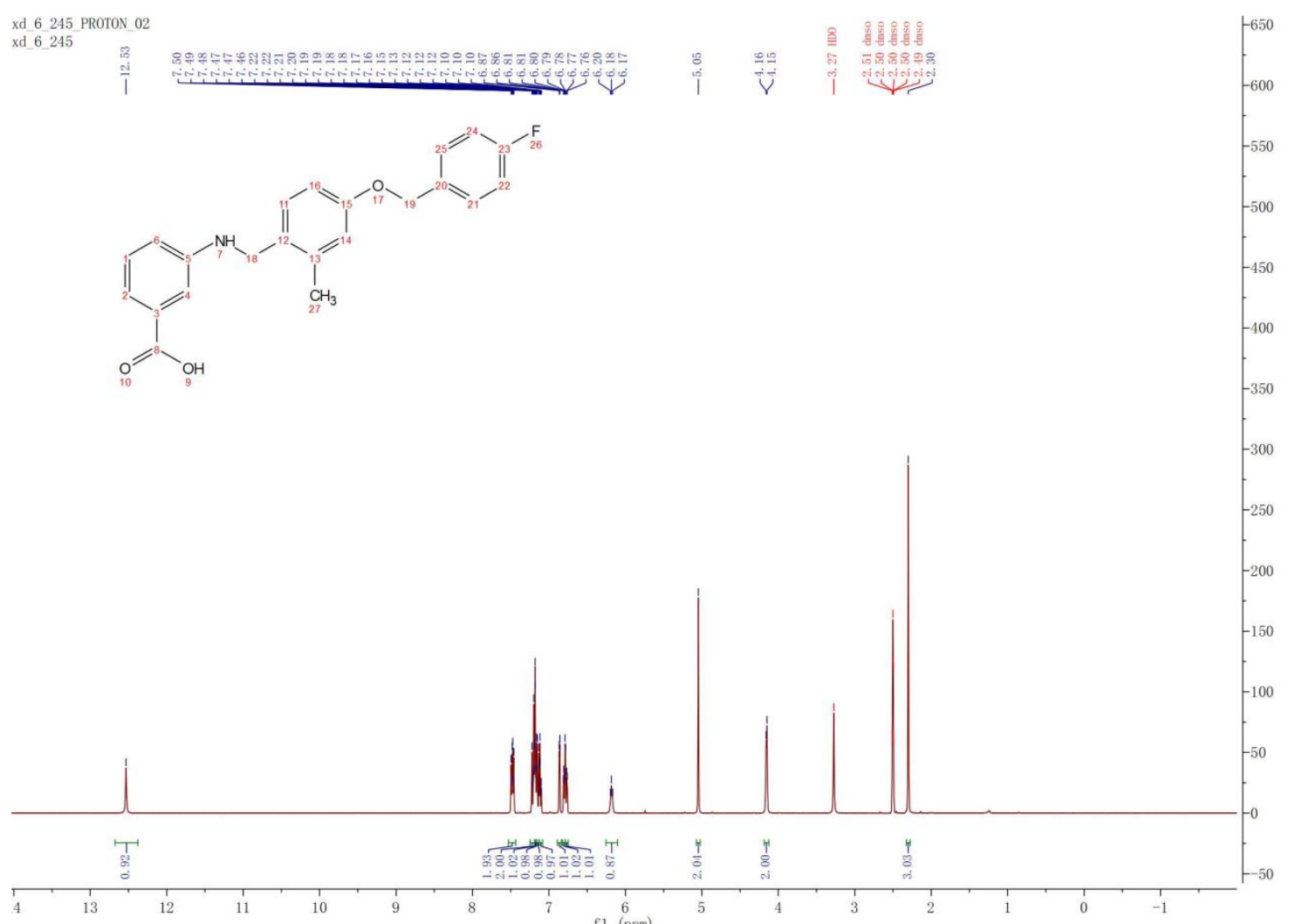

wasument xd 6245
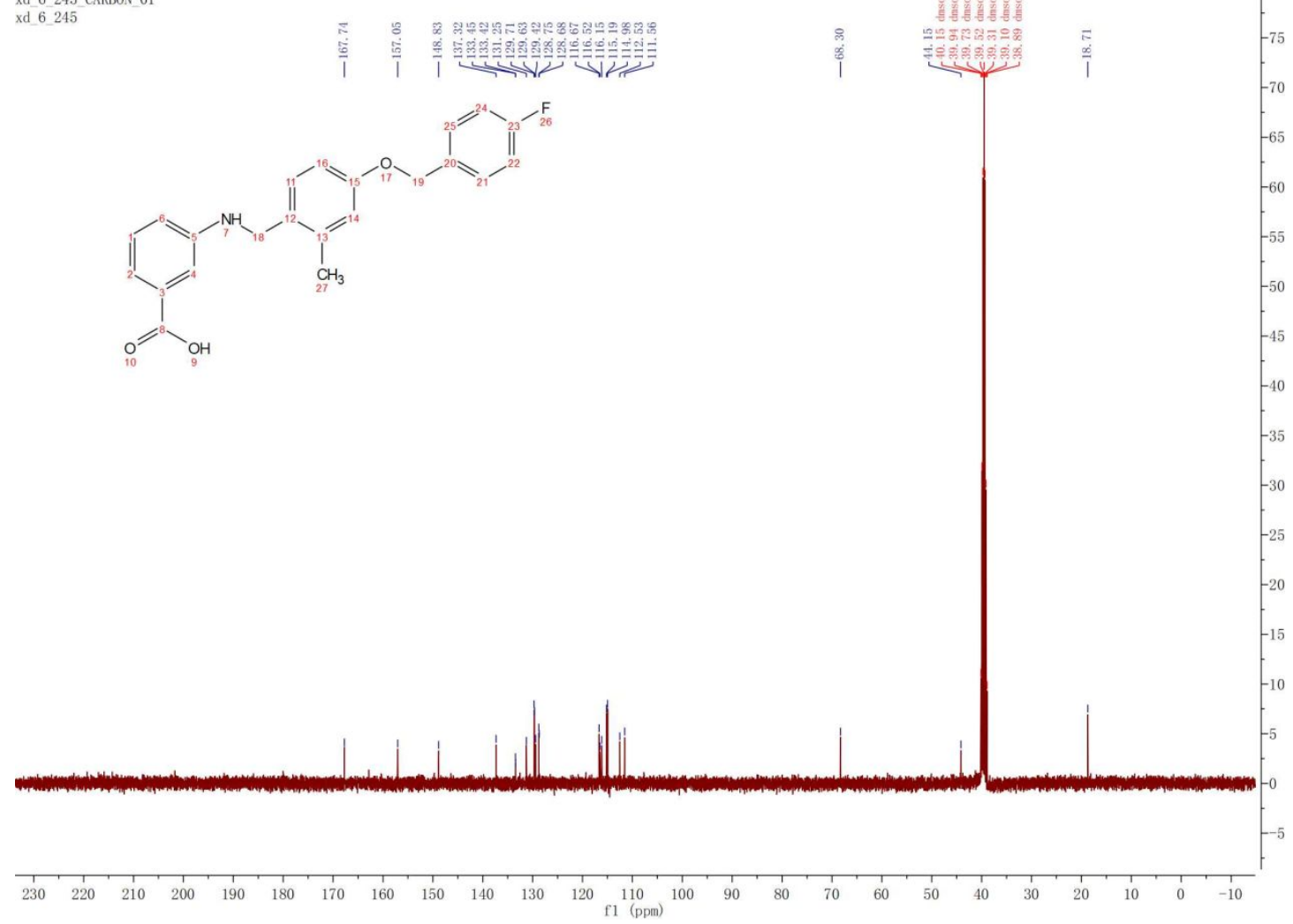
$4 u$

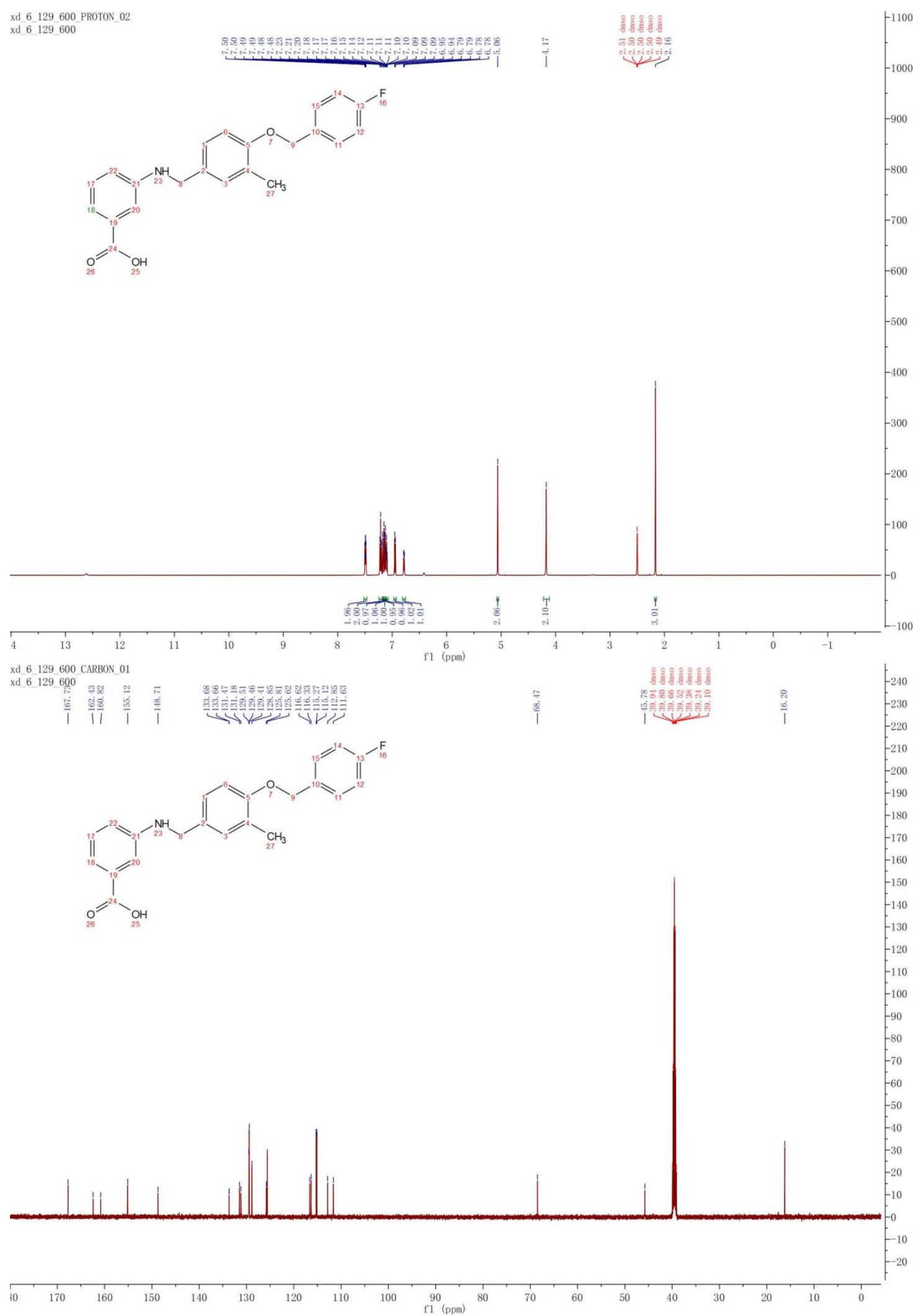


$4 \mathrm{~V}$

DN-5-45-i_PROTON_01
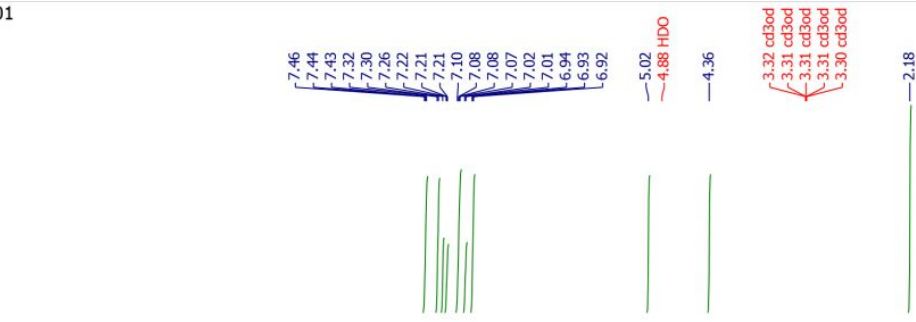

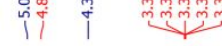

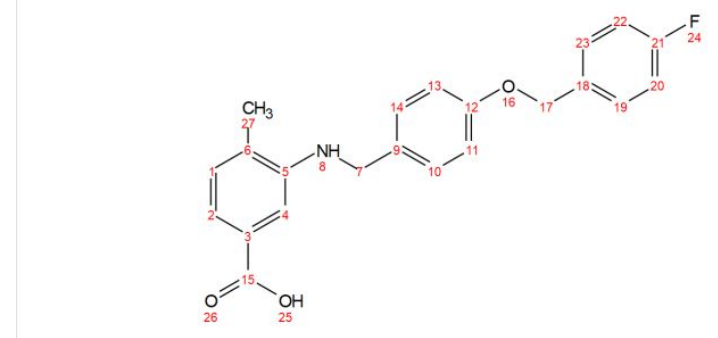

$-700$

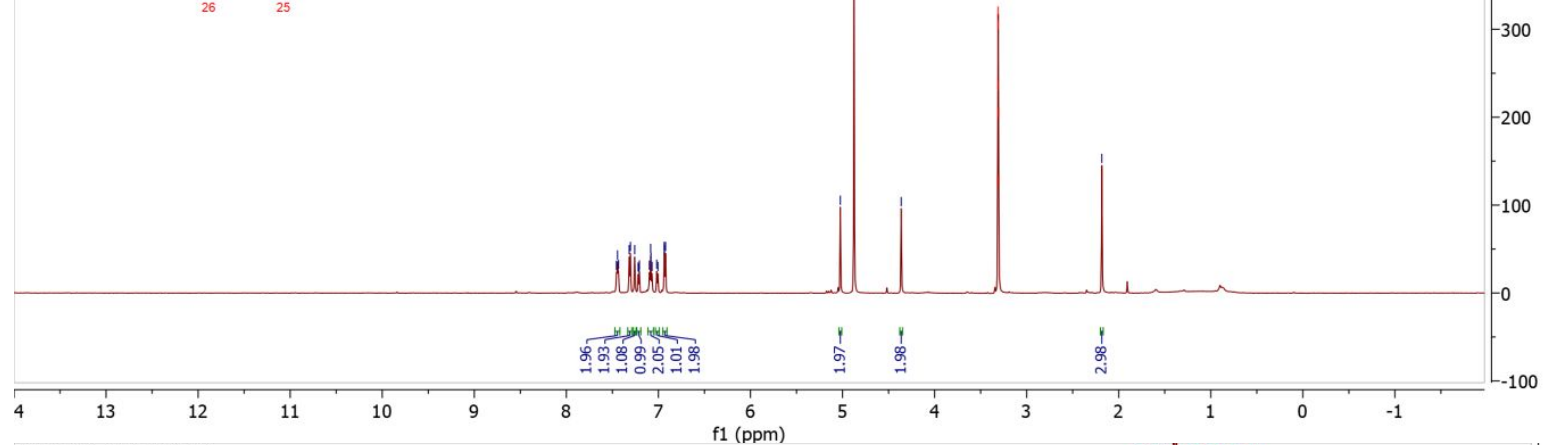
DN-5-45-i_CARBON_01

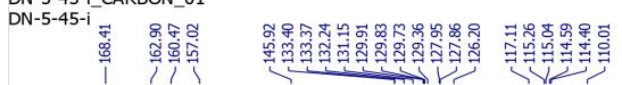

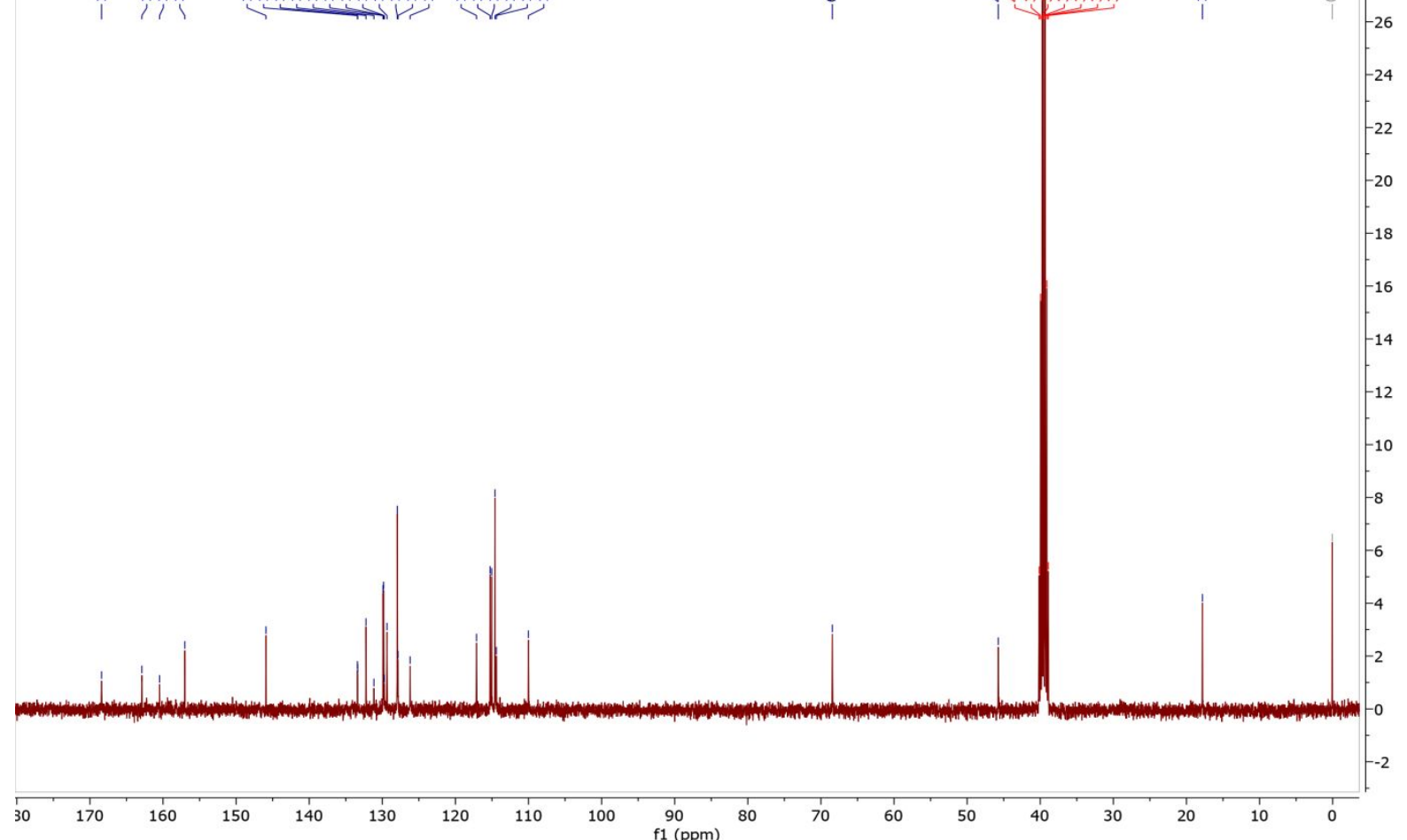

S30 


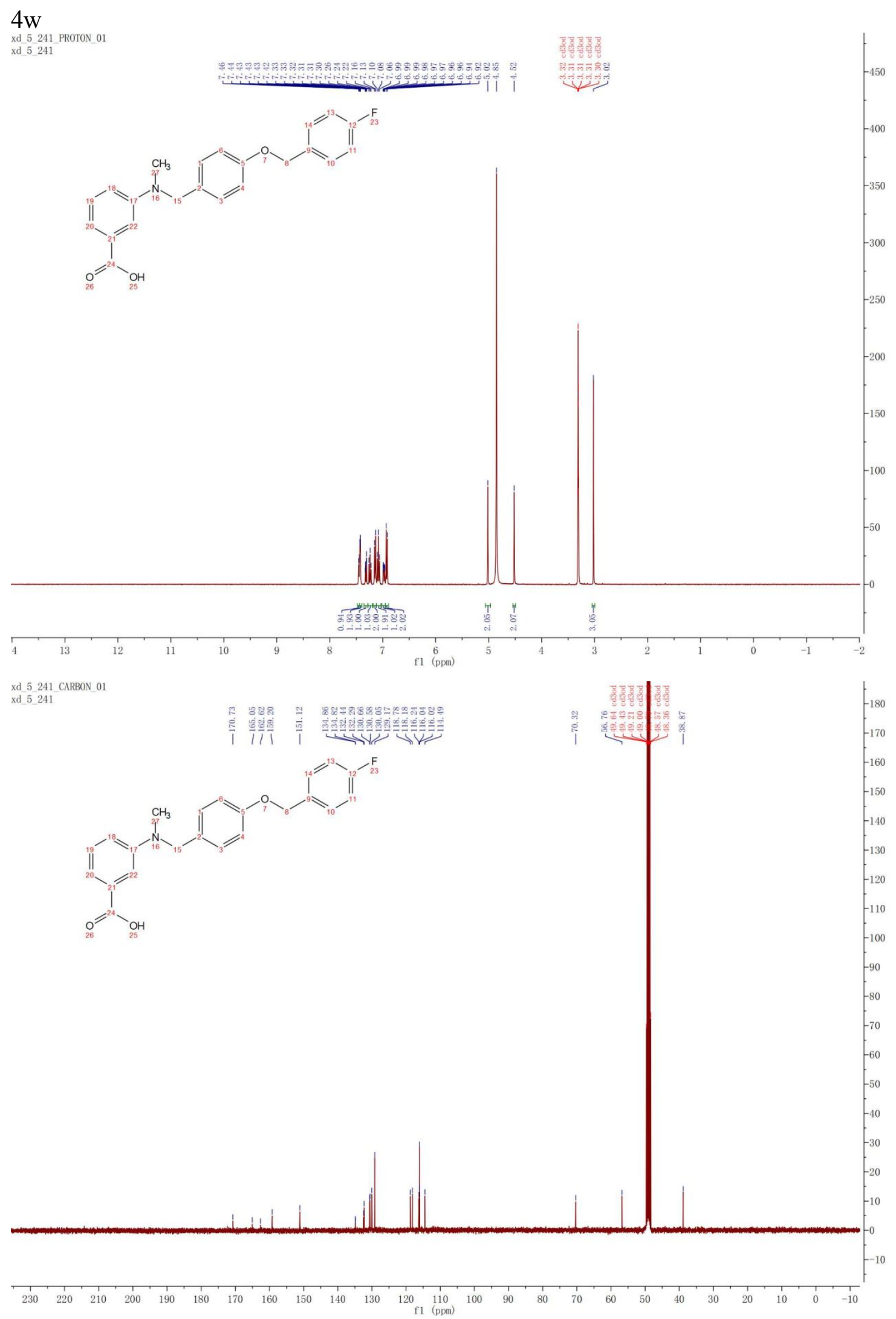

S31 
$4 \mathrm{x}$

$\begin{array}{lll}\text { xd } 6 & 251 \text { PROTON } 01 \\ \text { xd } 6 & 251\end{array}$

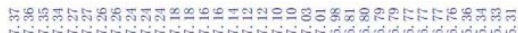

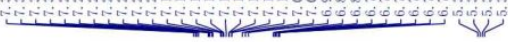<smiles></smiles>
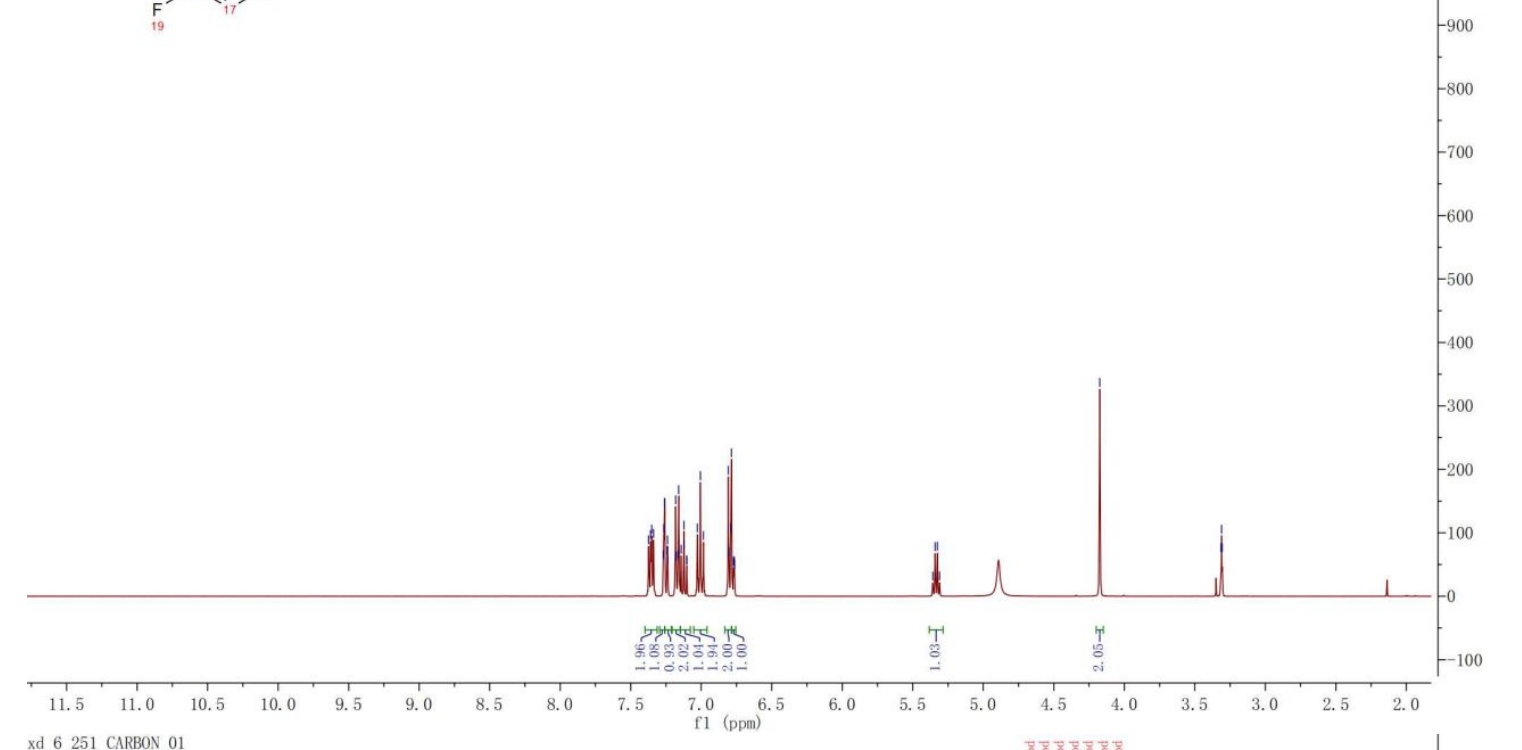
xd 6
xd 6 251 CARBON 01$$
\text { xd } 6251
$$

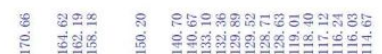

1 1) । Y<smiles></smiles>

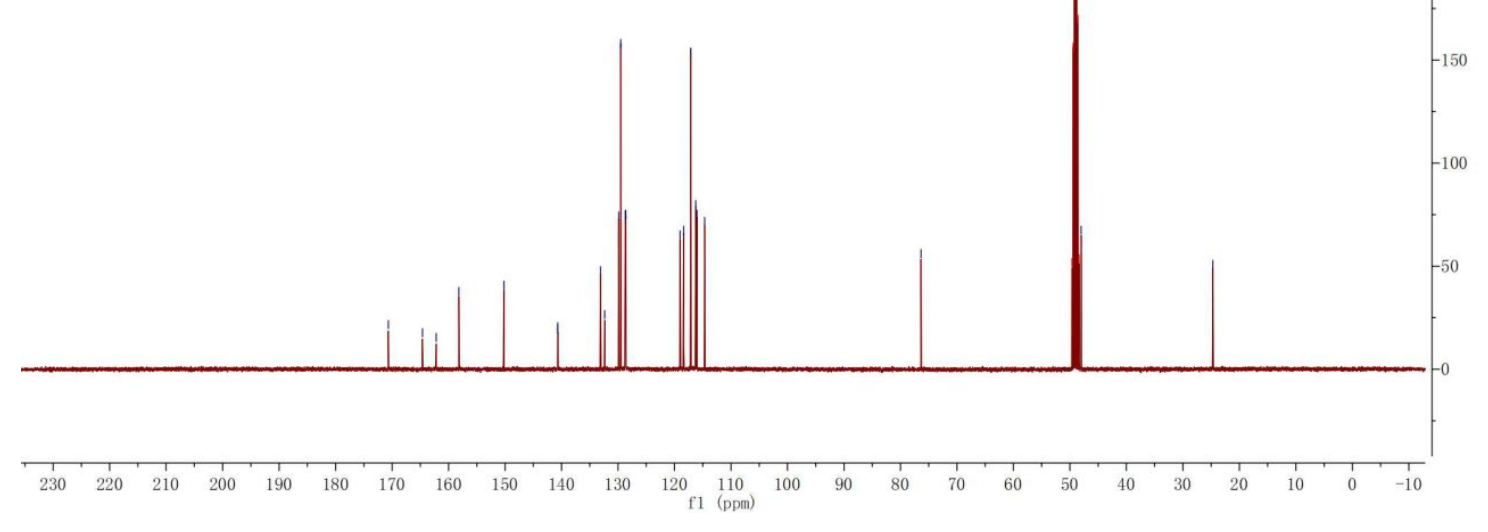

S32 


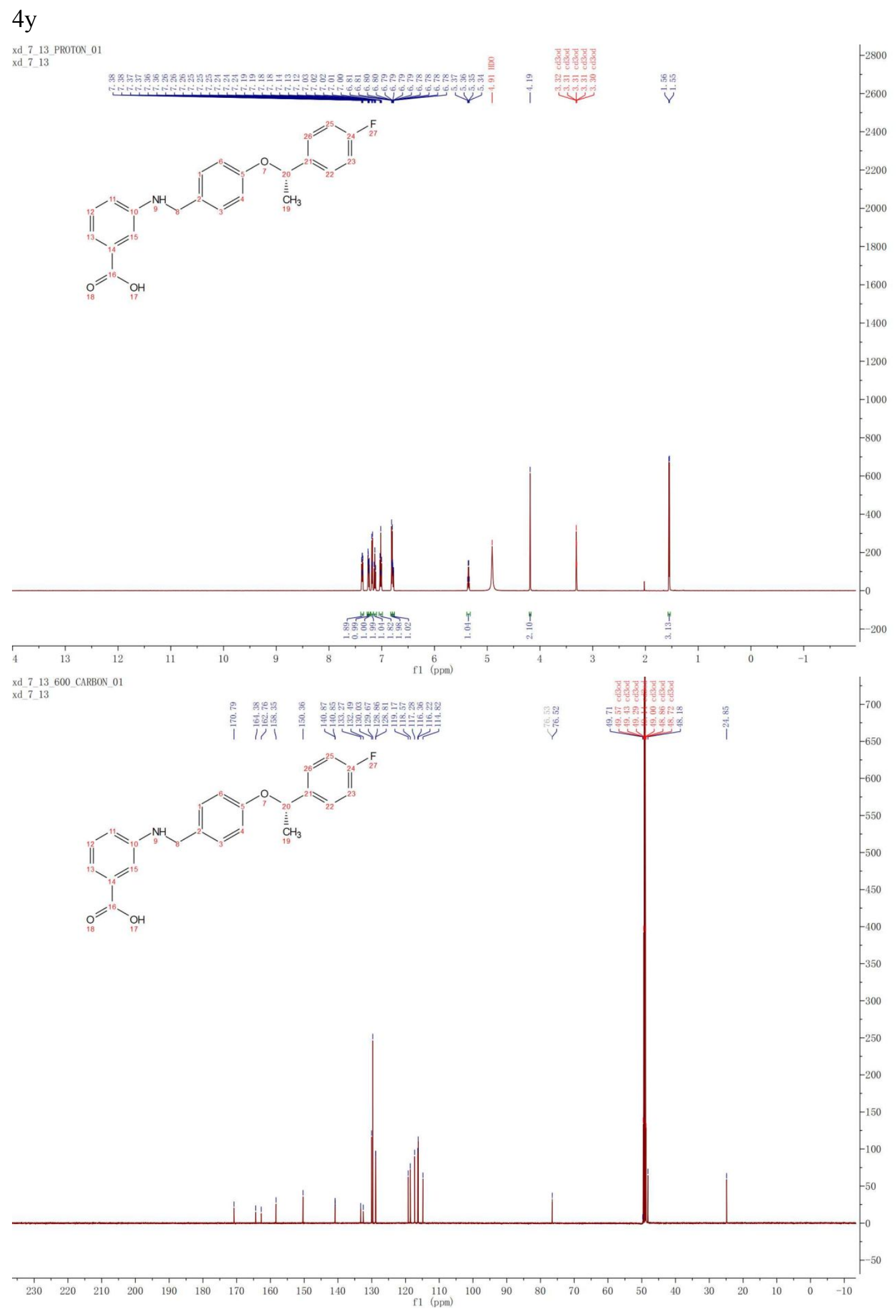

S33 


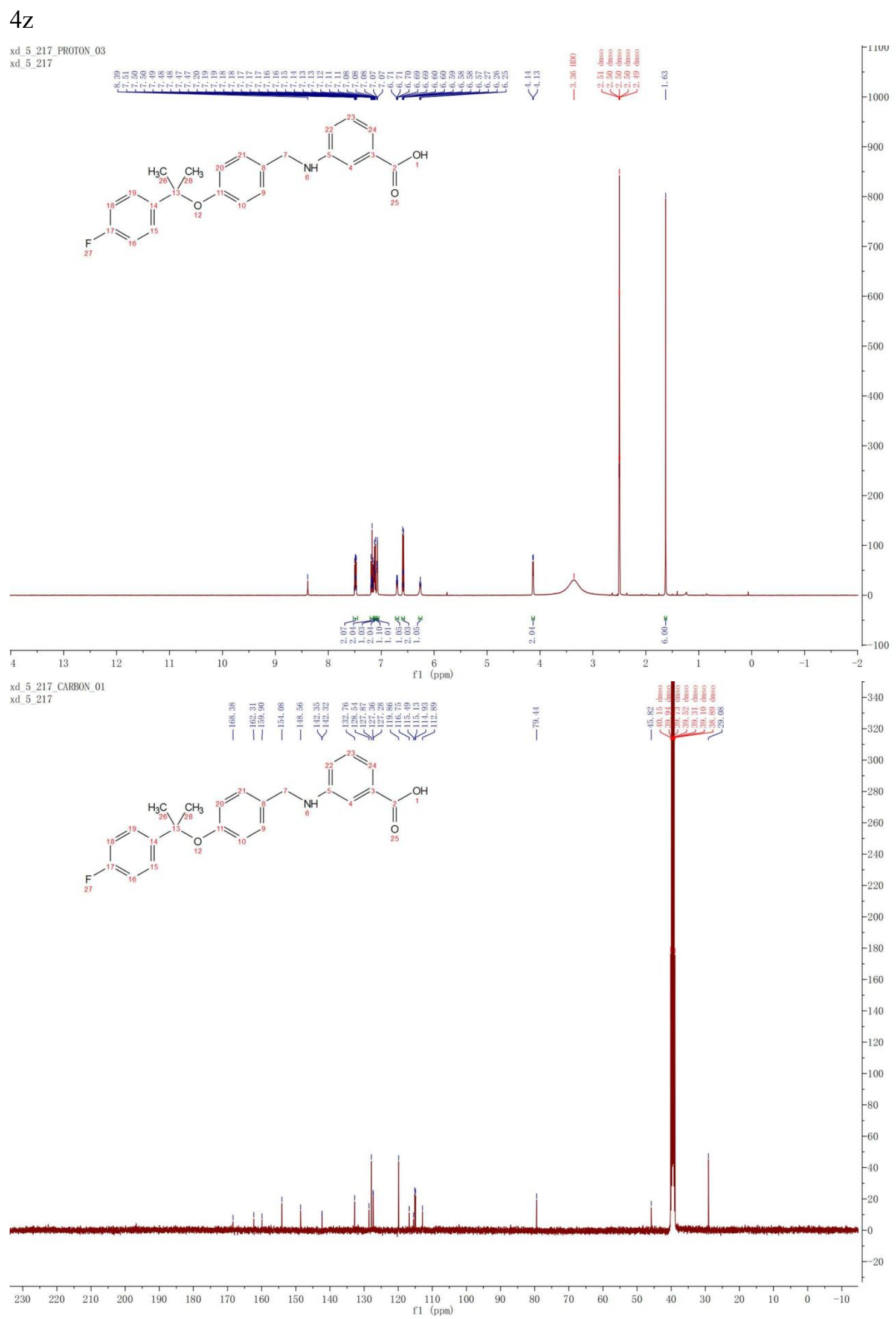

S34 
$5 \mathrm{a}$

Xd_4_211_PROTON_01

xd_4_211

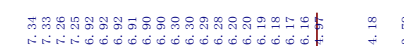

Ч

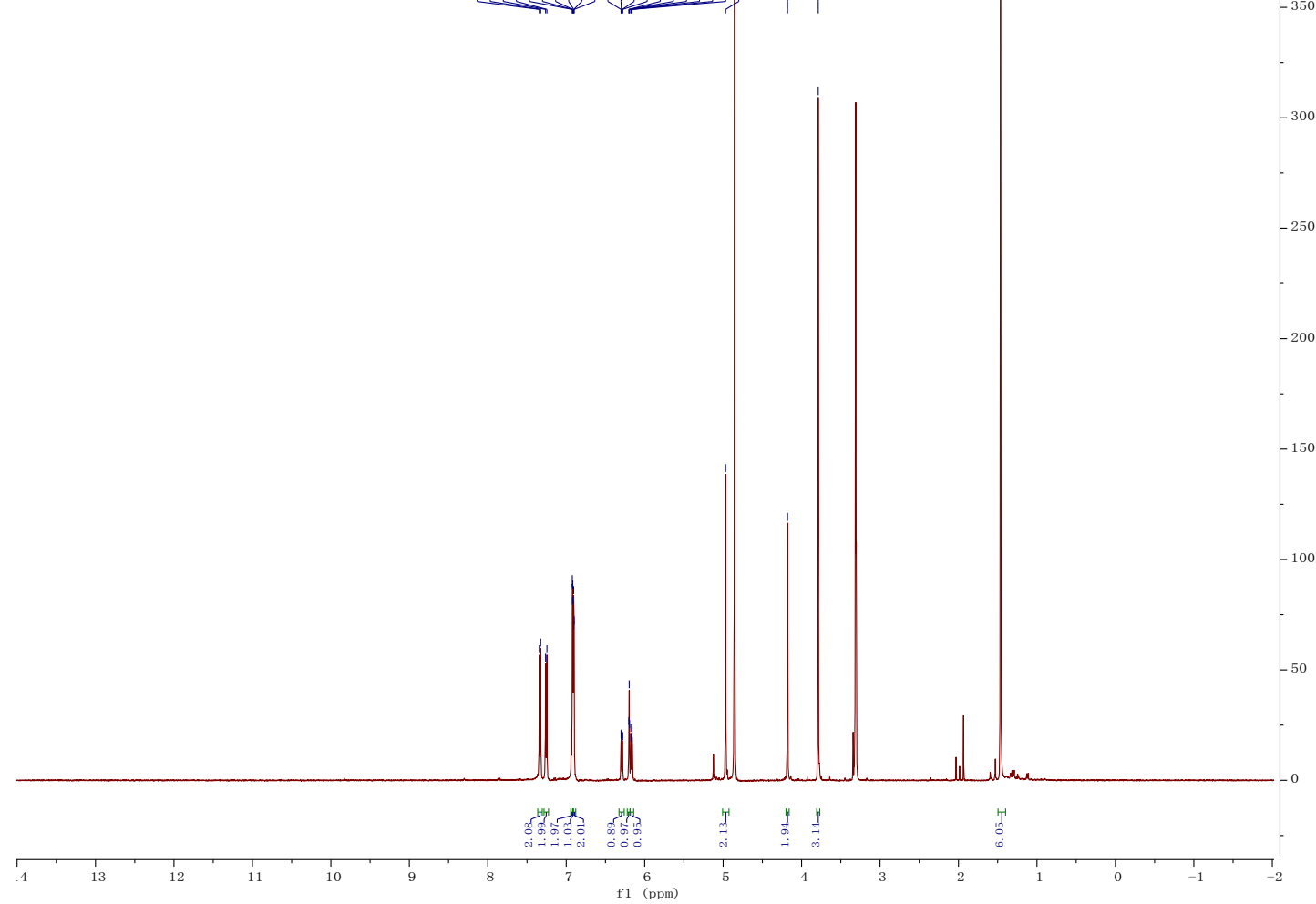

xd_4_211_CARBON_01
xd_4_211

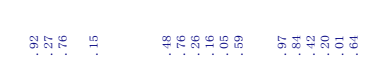

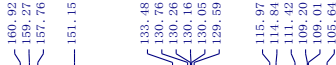

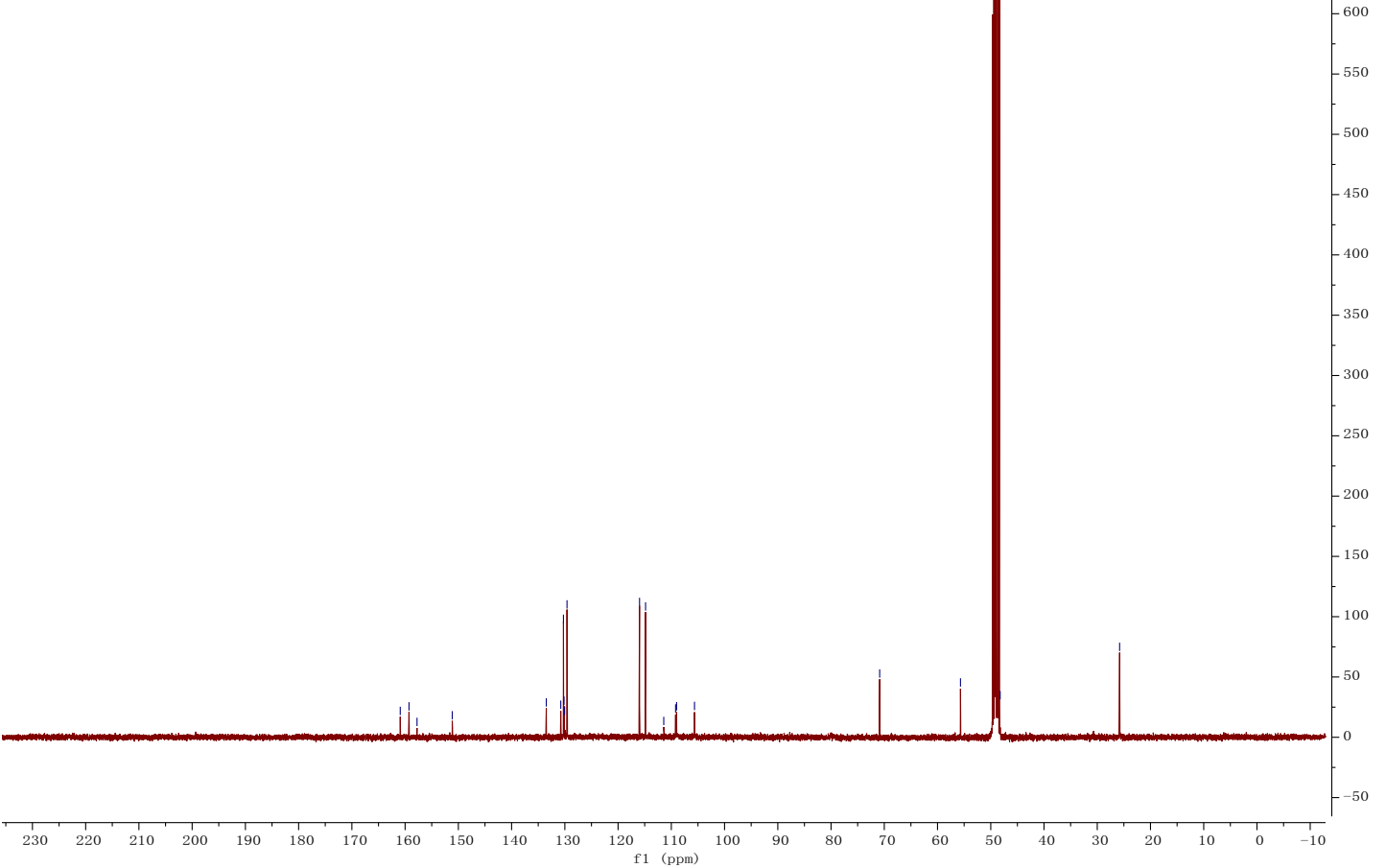

S35 
$5 b$

xd 4 271 600 PROTON 02

xd $4 \_271 \_600$

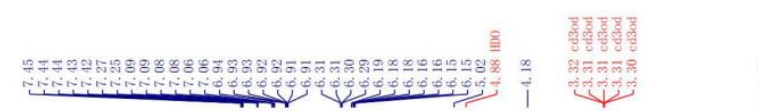

$\stackrel{-4000}{i}$<smiles>C[Pb](O)(Oc1cncnc1NCc1ncnc(OCc2ccc(F)cc2)n1)C(=O)O</smiles>

$-3500$
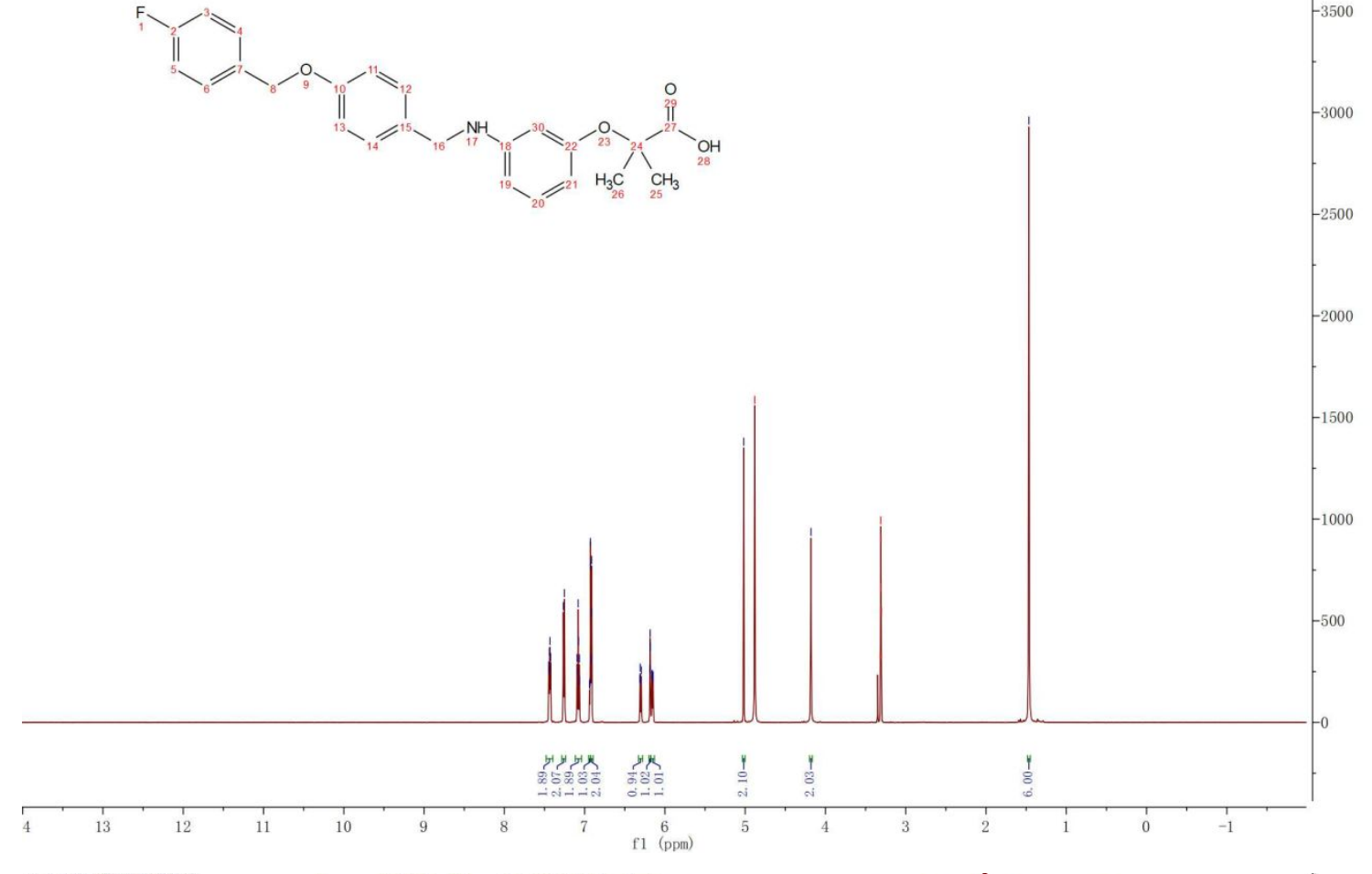

xd 4 $4271 \_600$ CARBON 01

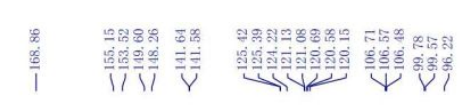

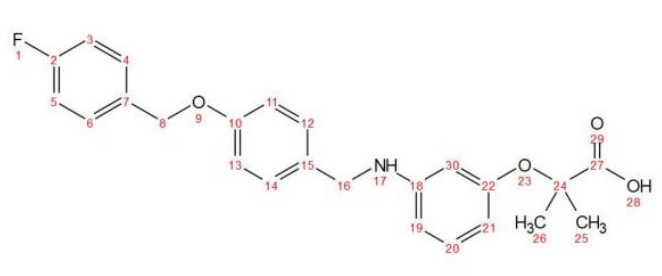

xd 4271600

\section{-01}

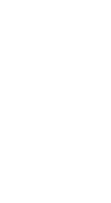

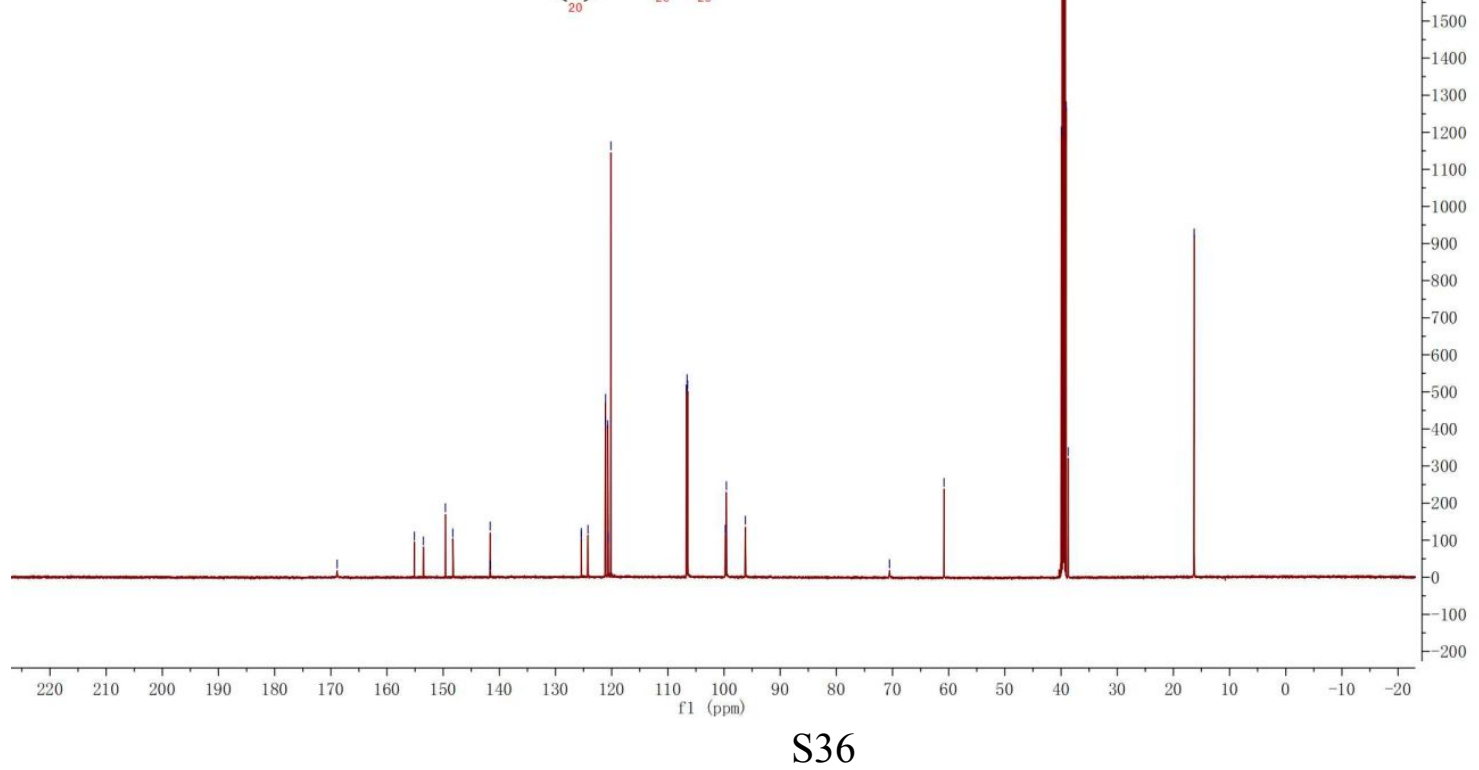




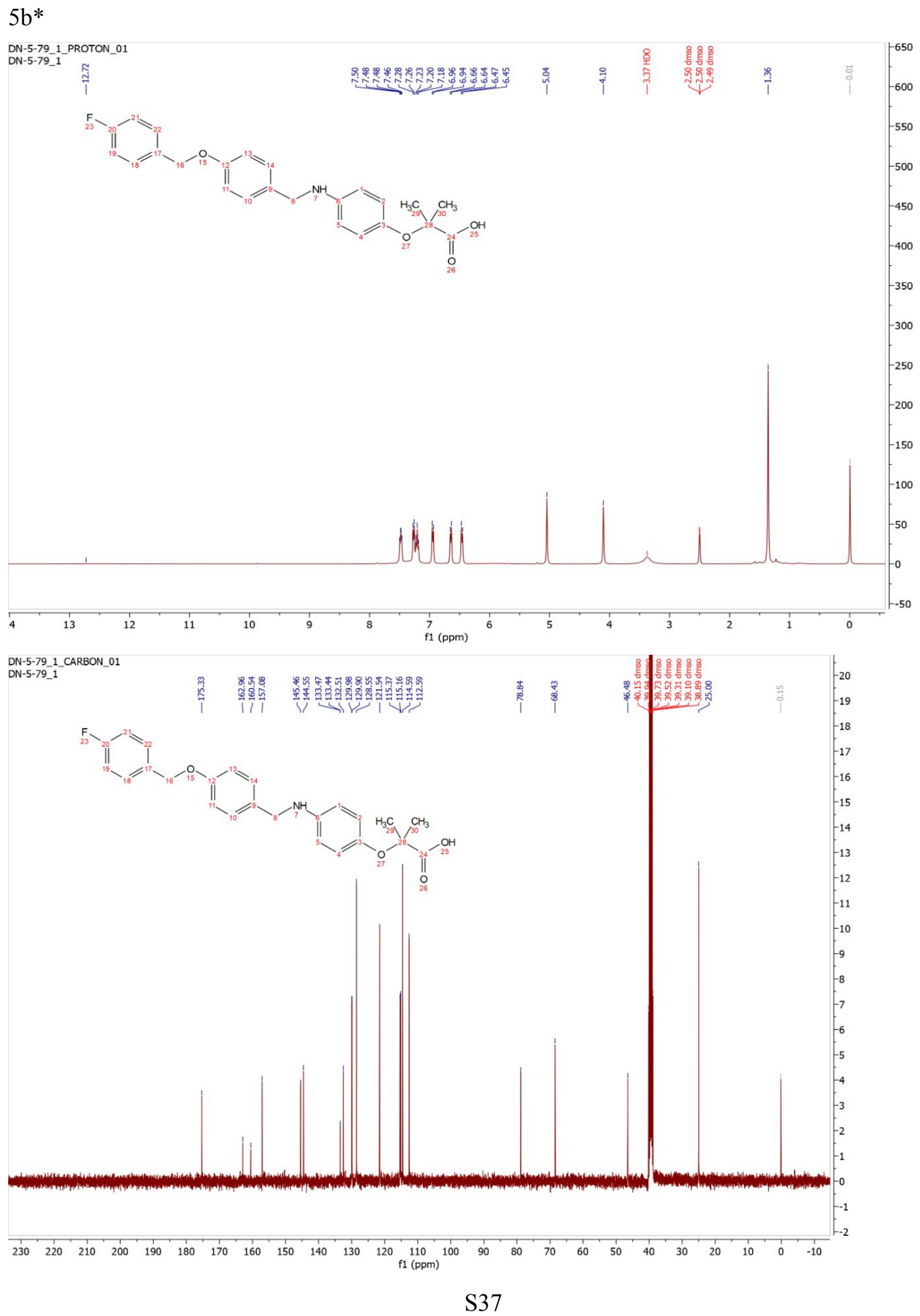




\section{$5 f$}

xd_4 279_carbon_PROTON 01

xd 4 279 carbon

98:

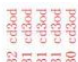

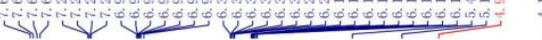

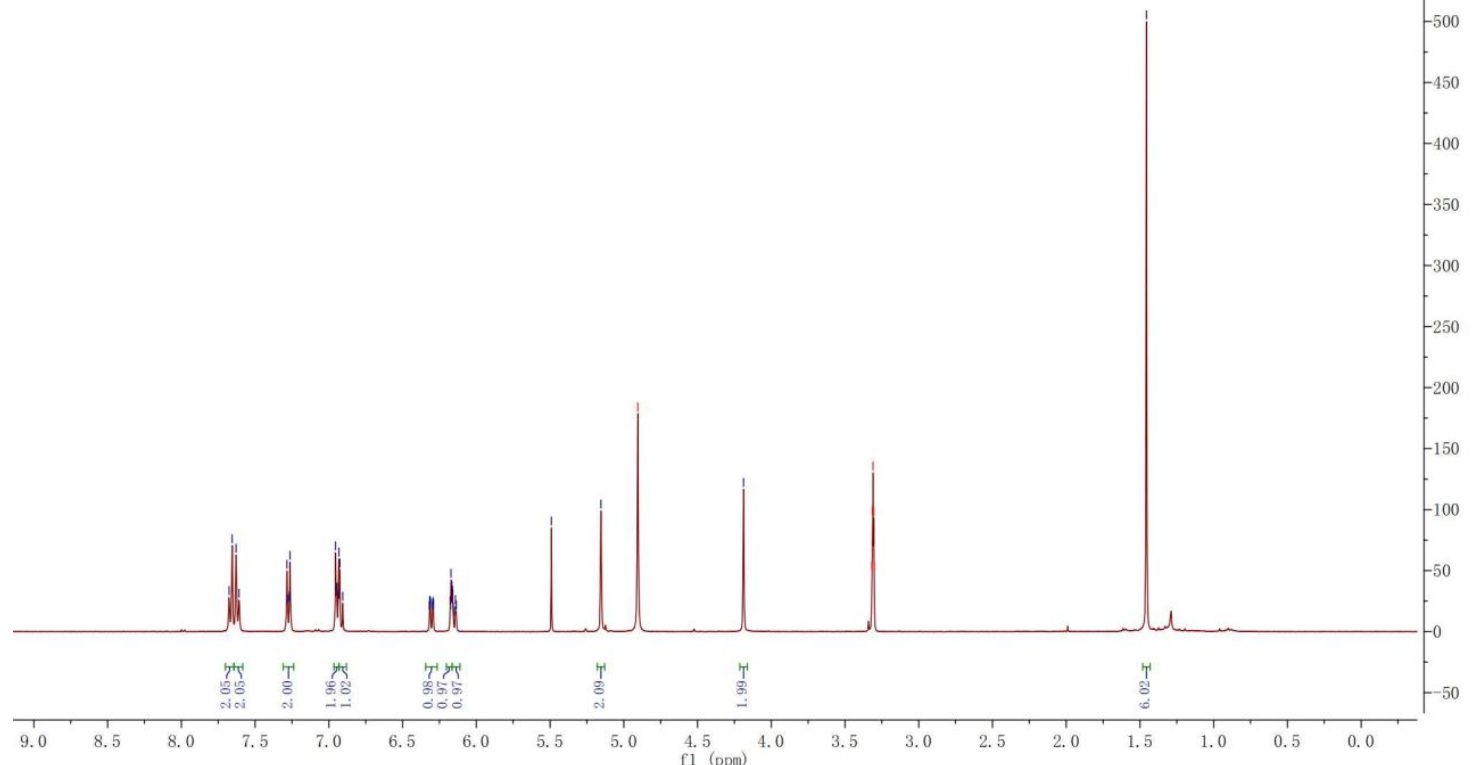

xd_4_279_CARBON_01 $x d$ _ 279
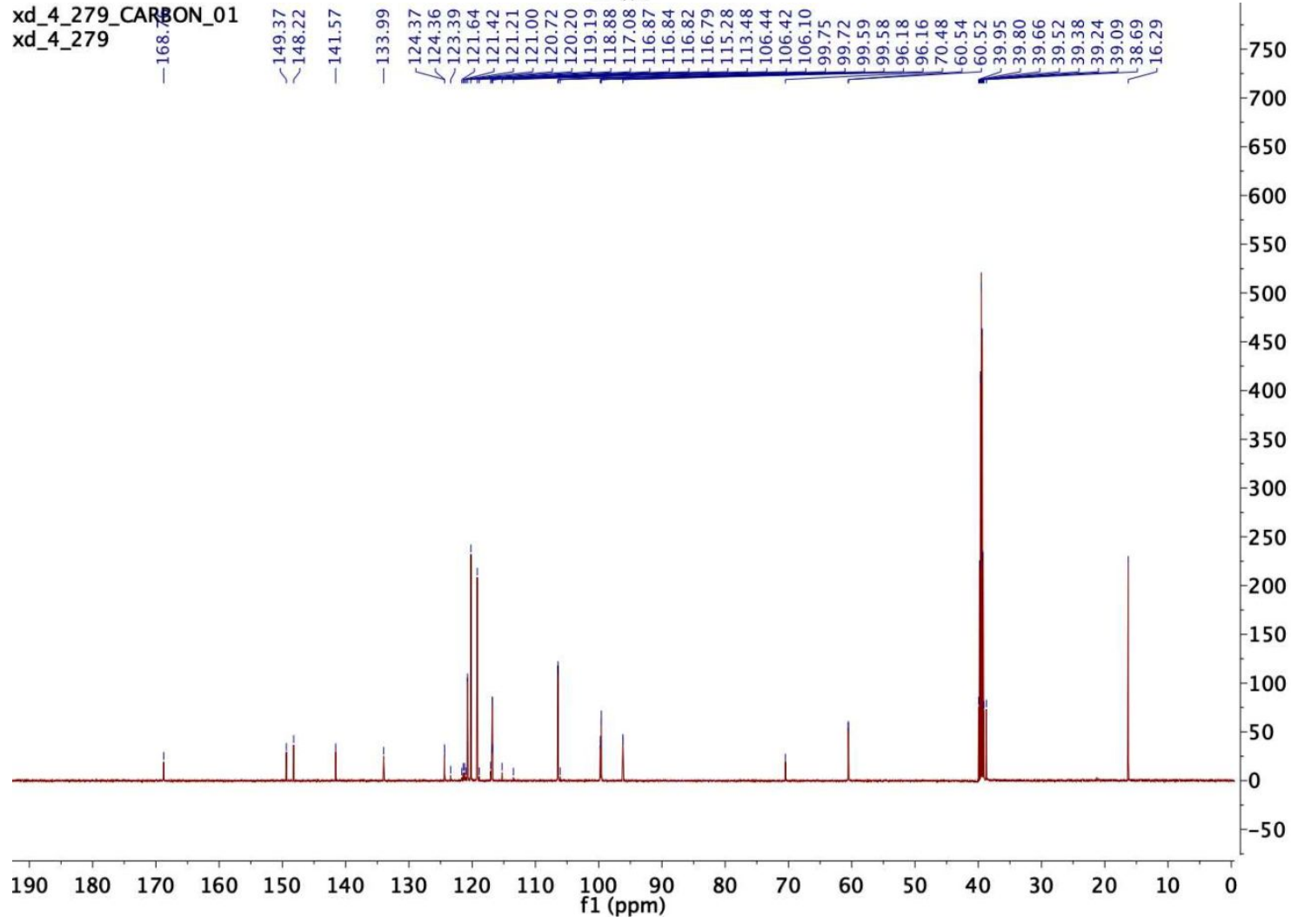

S38 
$19 \mathrm{a}$

xd 5 107_500 PROTON 02

xd 5_107_500

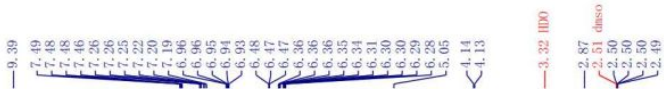

1200

\begin{tabular}{|l|l|}
-1100 \\
-1000
\end{tabular}
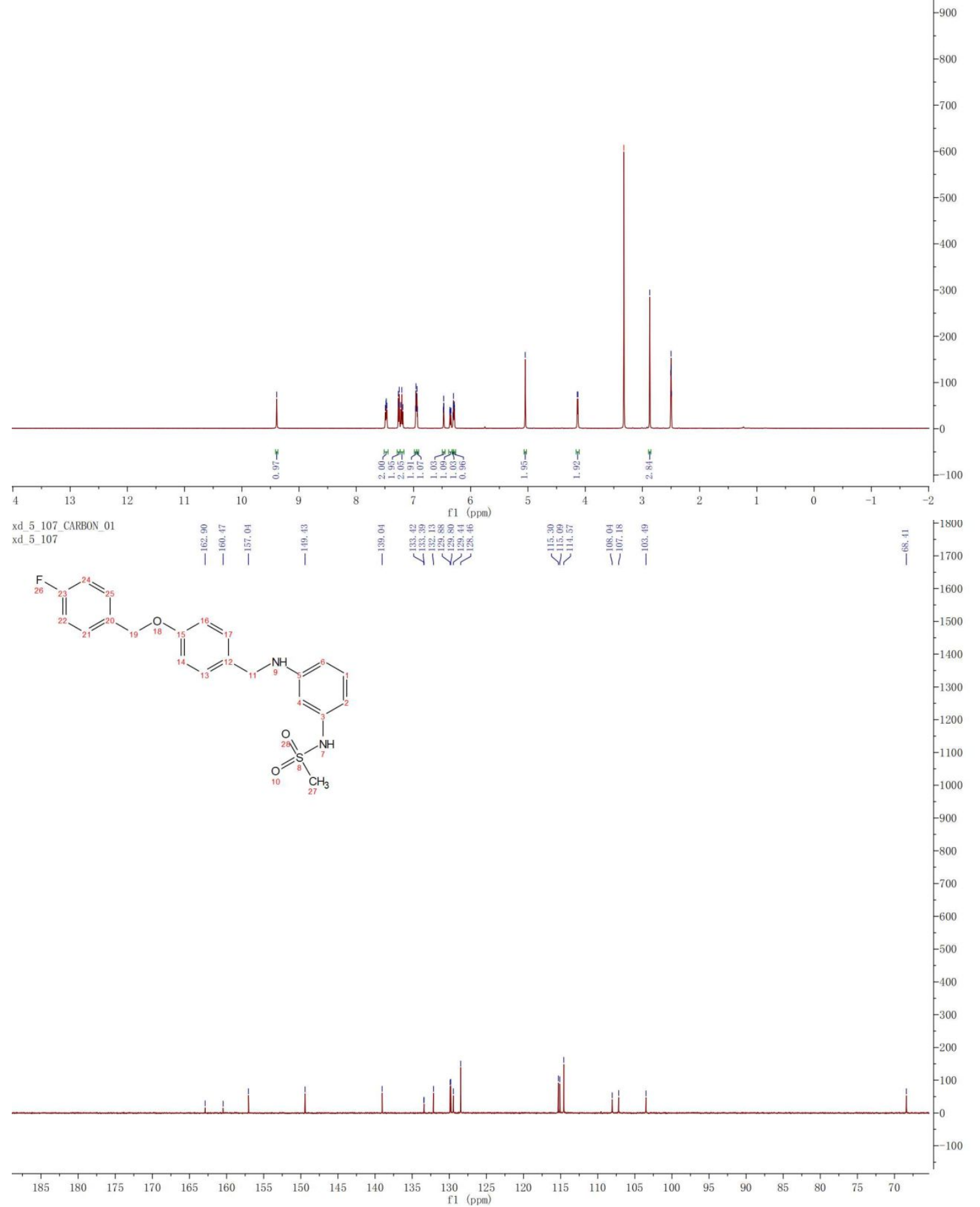

S39 


\section{$19 b$}

xd 5 109 PROTON 01
xd 5 109

aN 01

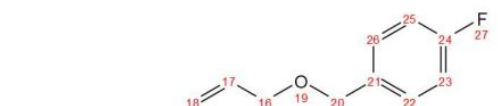

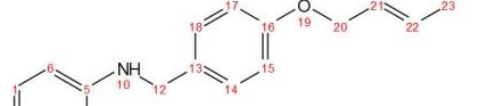

$\prod_{3}$

$=2 \mathrm{NH}^{\circ} \mathrm{OH}^{11}$

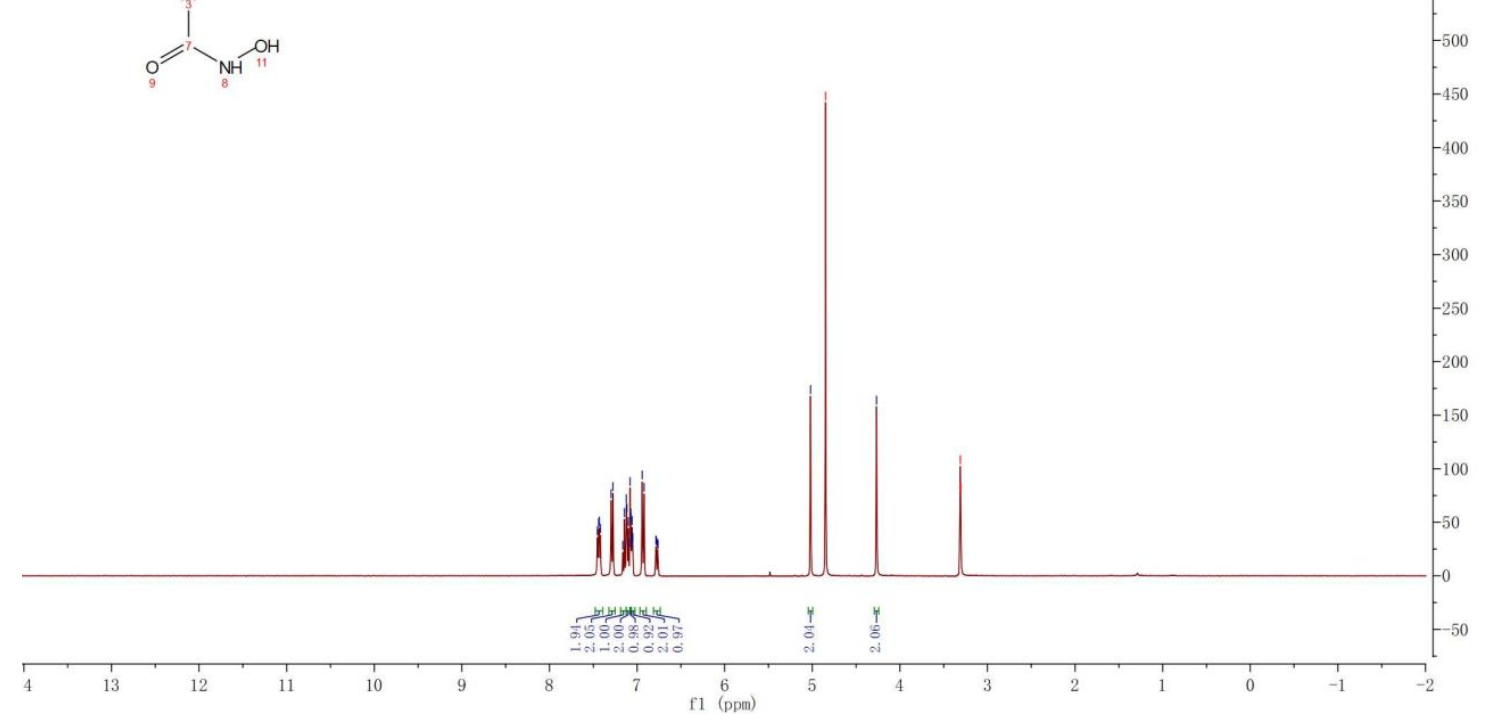

xd 5 109 CARBON 01 $\begin{array}{lll}x d & 5 & 109\end{array}$

10 9요

।
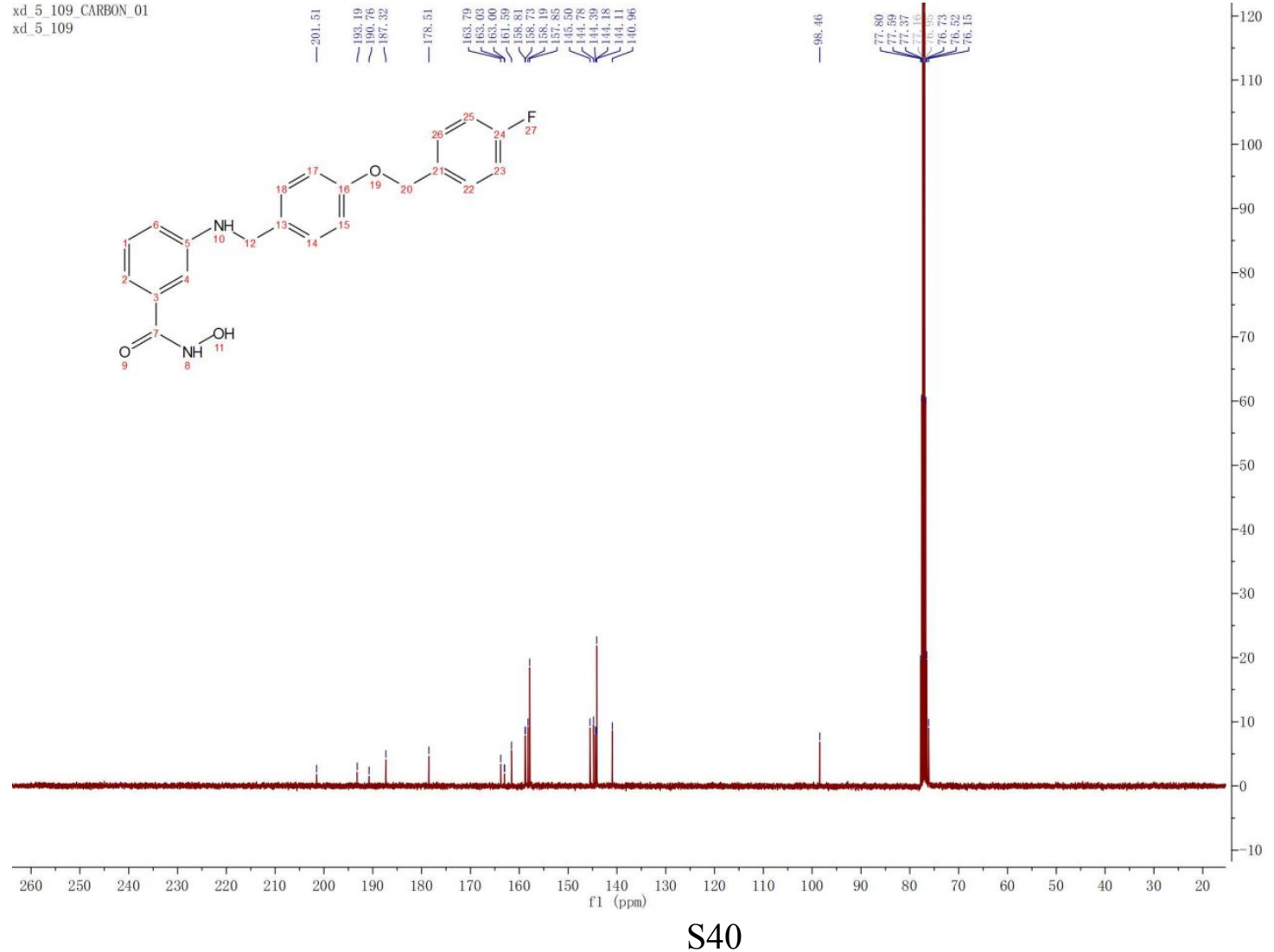


\section{9c}

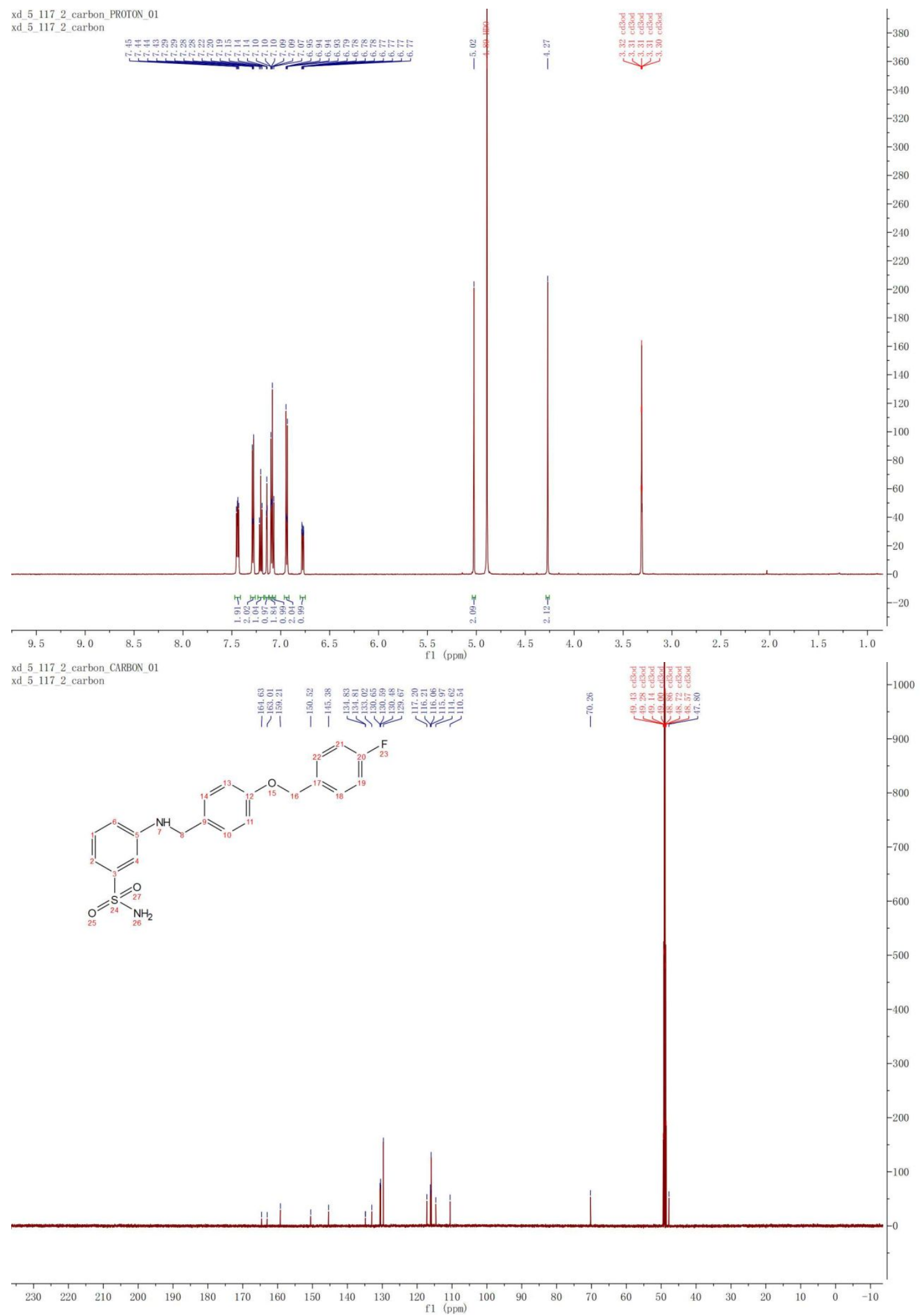


19d

xd 5 219 PROTON 01

$x d \quad 5 \quad 219$
xd $5 \quad 219$

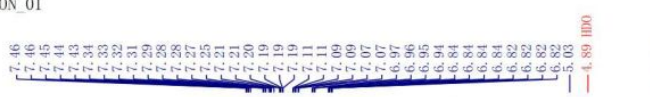

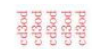

해요

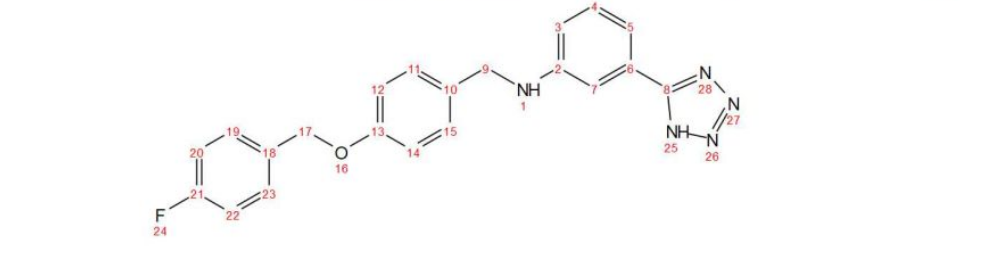

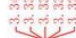

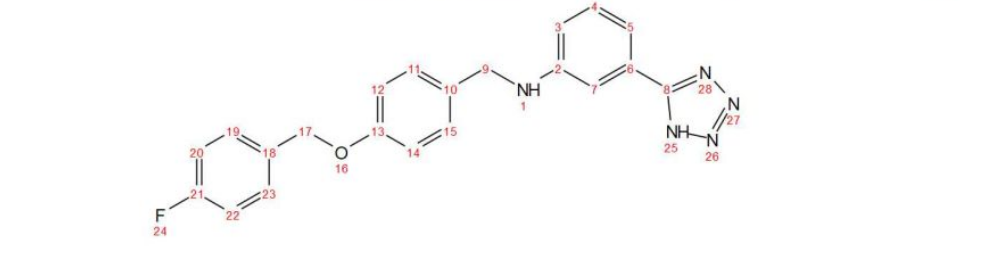

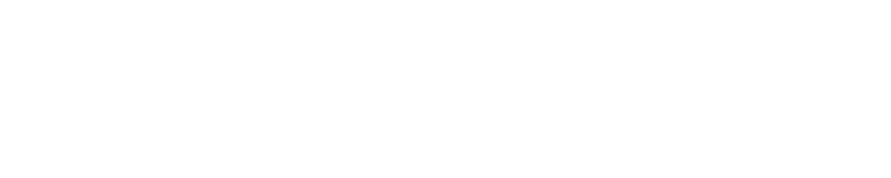

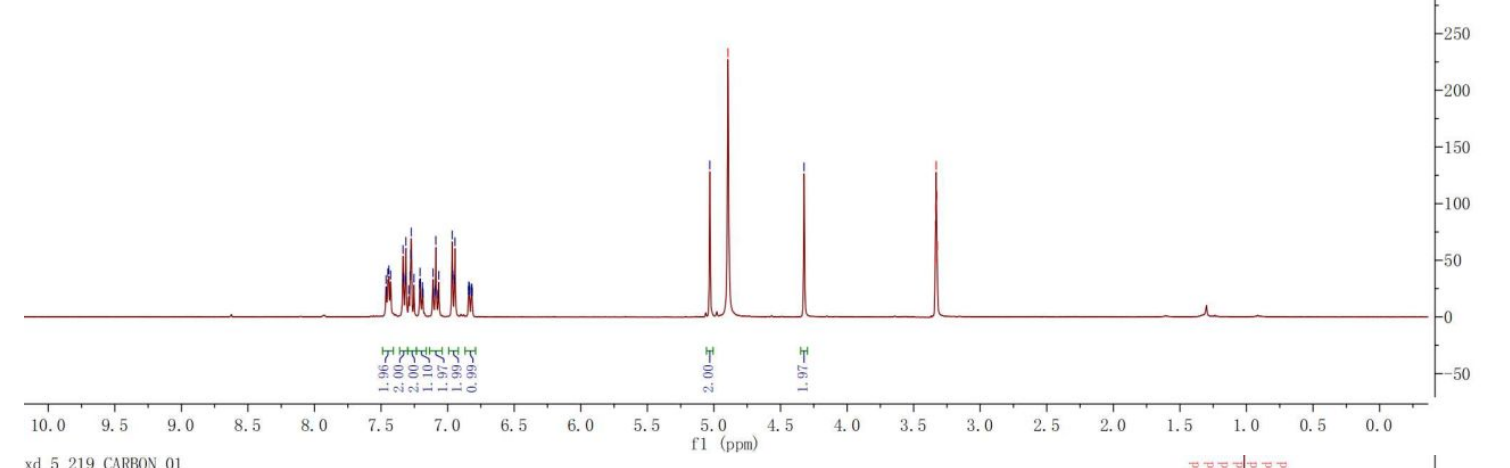

xd 5219 CARBON 01 xd 5219
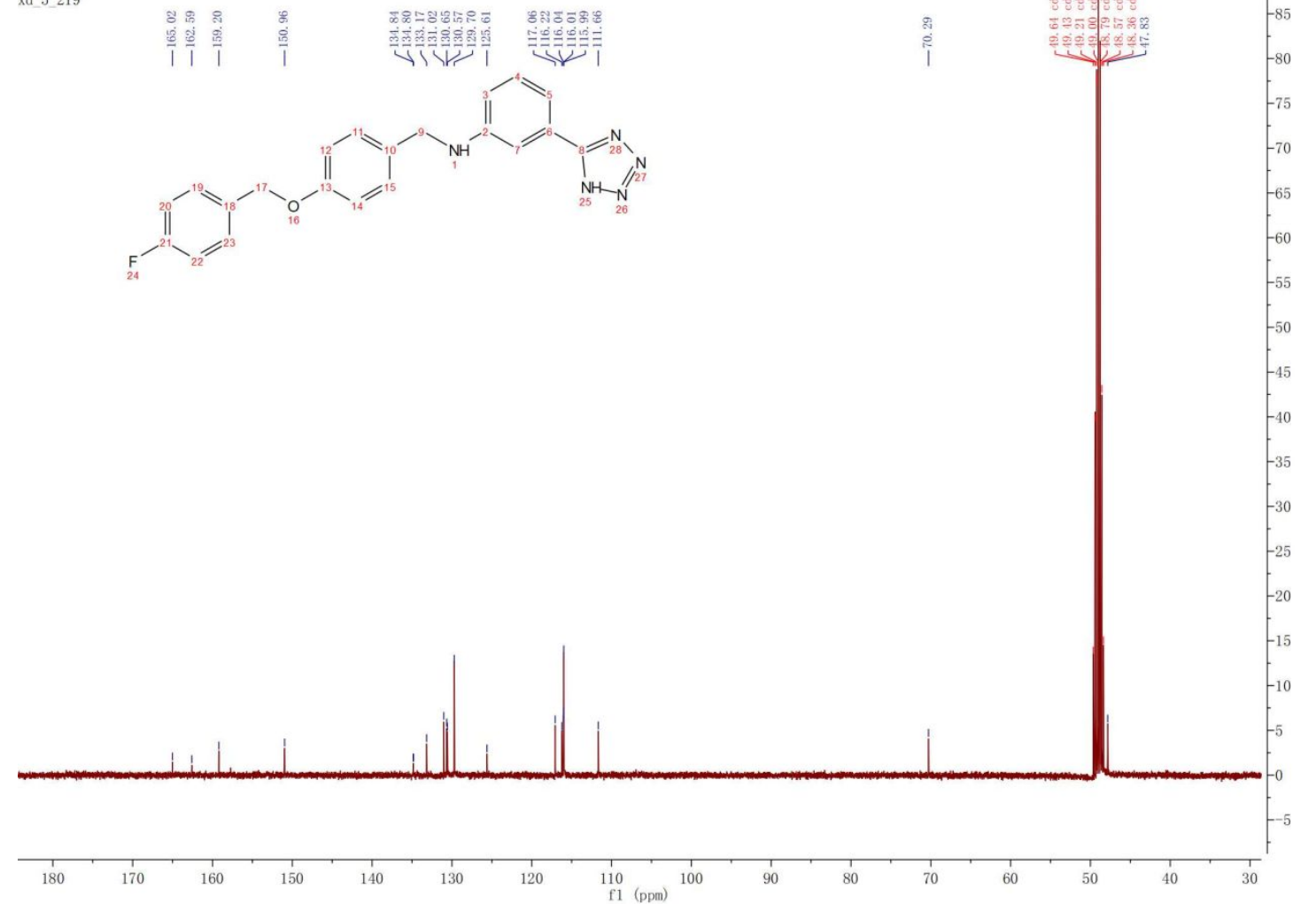

S42 


\section{9 e}

xd 6 29 P Methanol PROTON 01

xd 6 29 P Mlethano
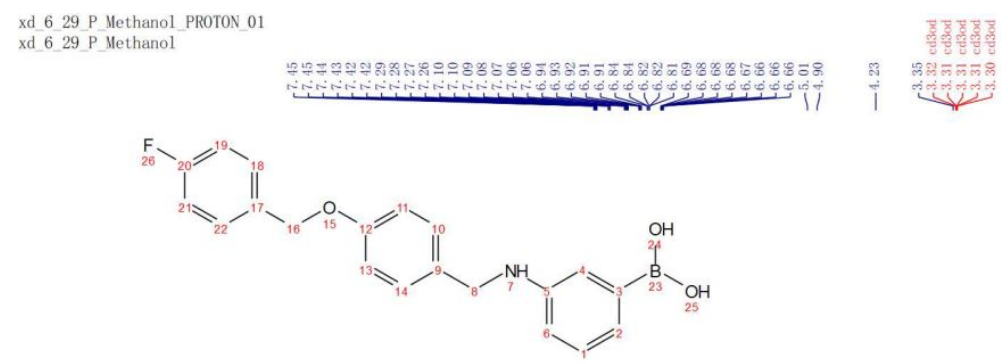

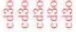

4

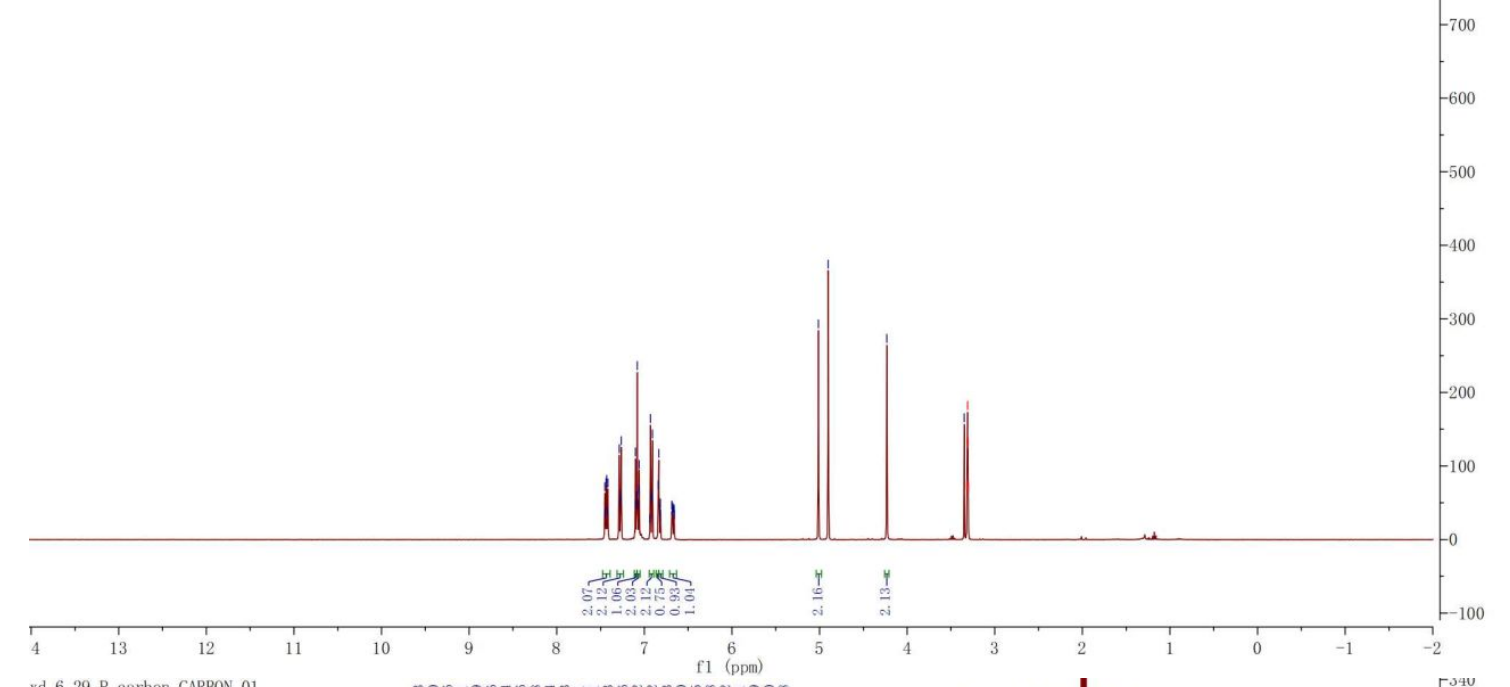

xd 629 P_carbon_CARBON 01

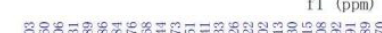

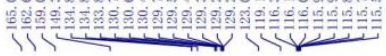

xd 629 P carbor
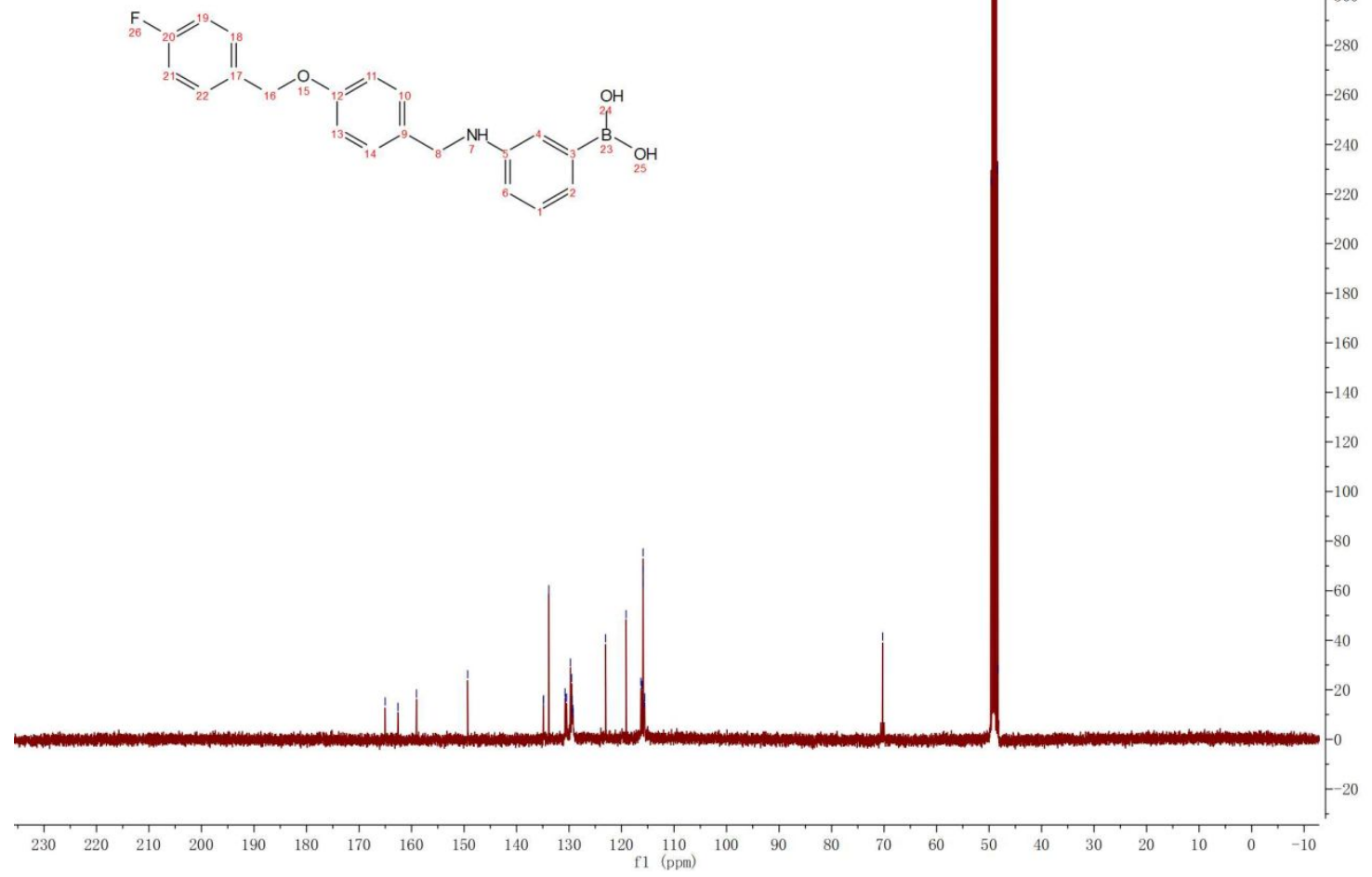

S43 


\section{$19 f$}

xd 6 6 101_PROTON 01

xd $6 \_101$

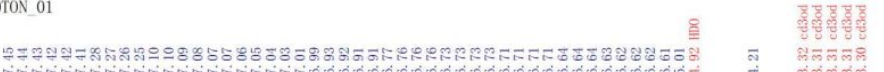

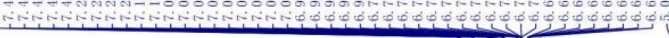

$-2200$

$-2100$

2000

1900

1800

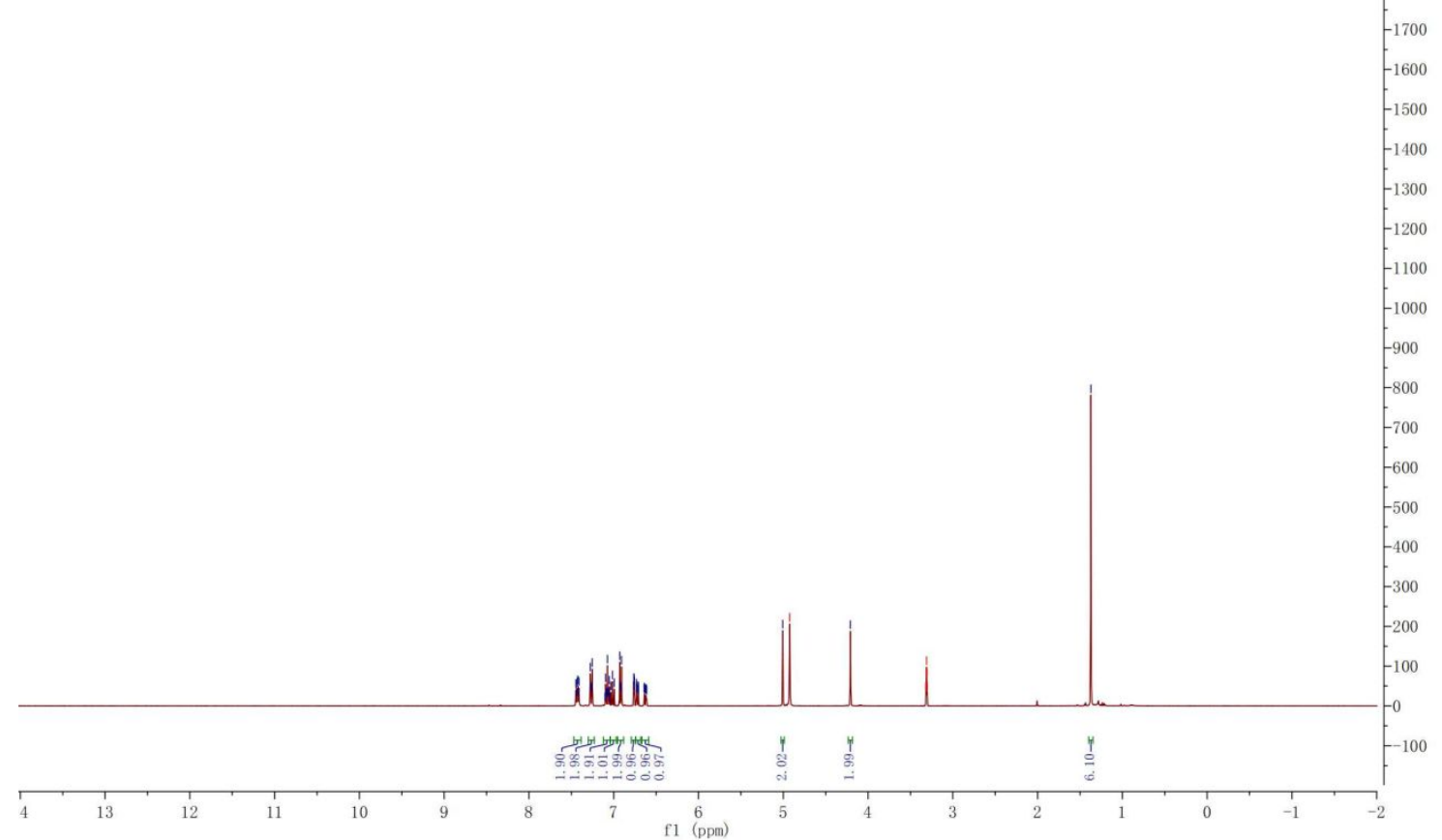

xd 6_101_CARBON 01

xd 6101
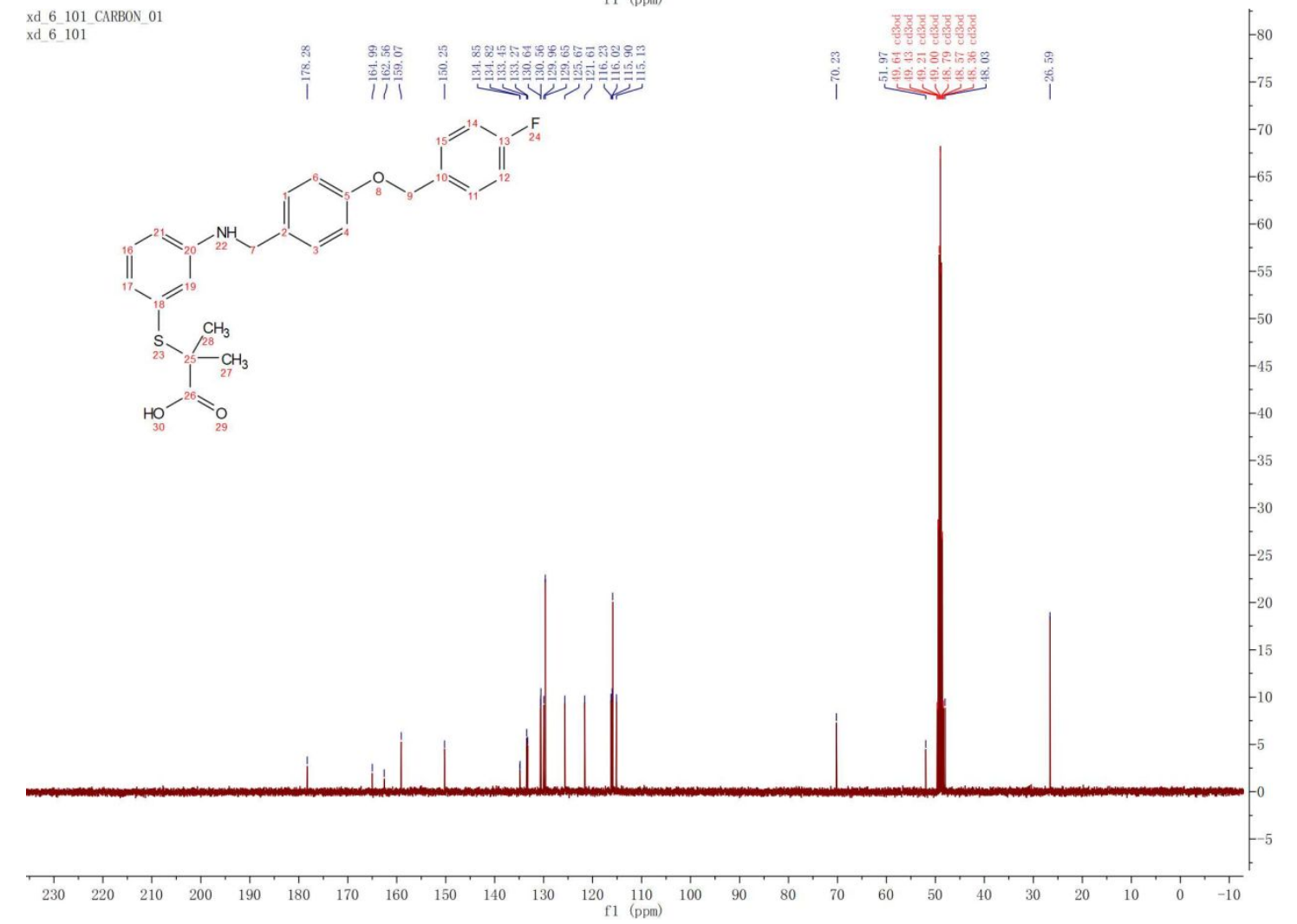

।

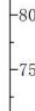

S44 
$\overrightarrow{0}$
a
।

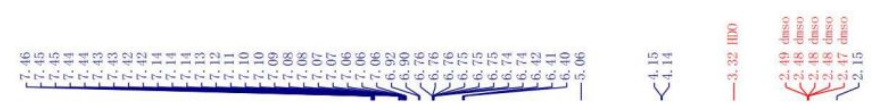

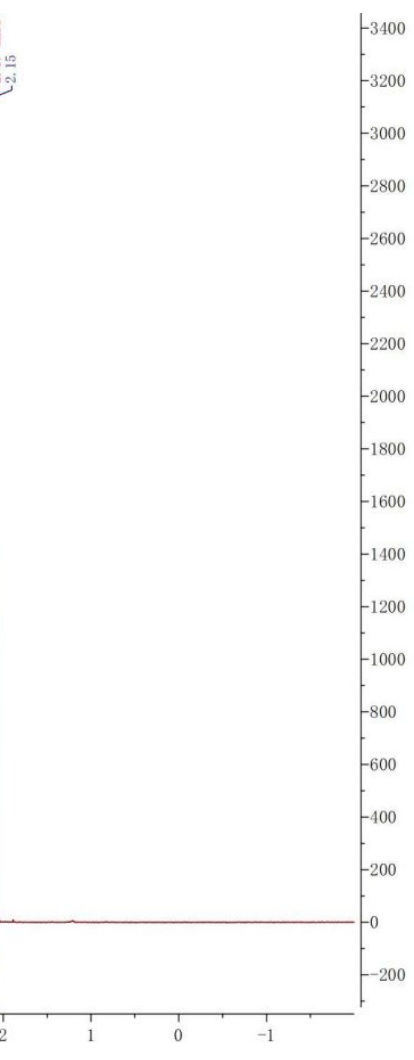

(2)
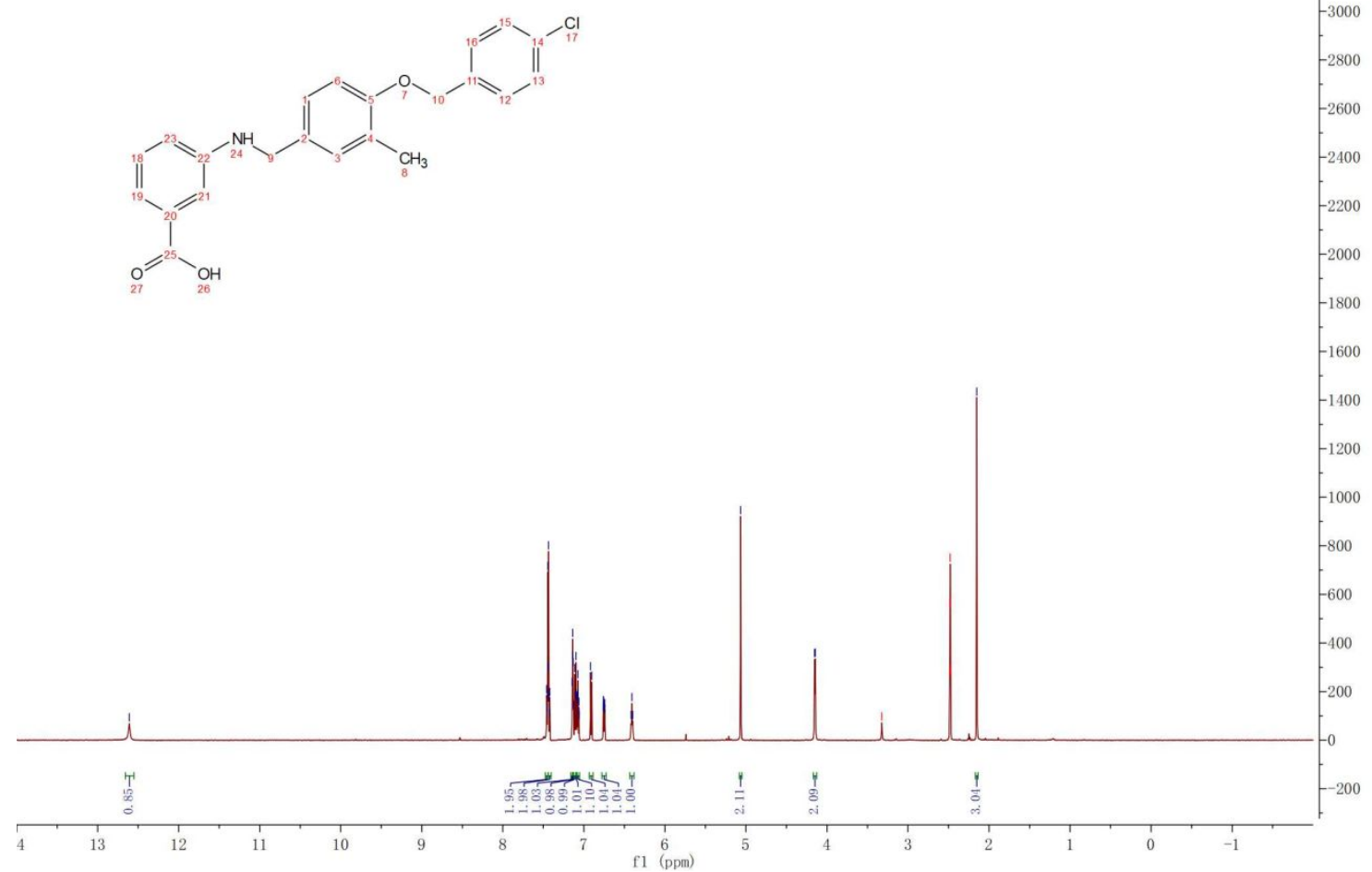

xd 749600 CARBON 01 xd 7_49_600

ल

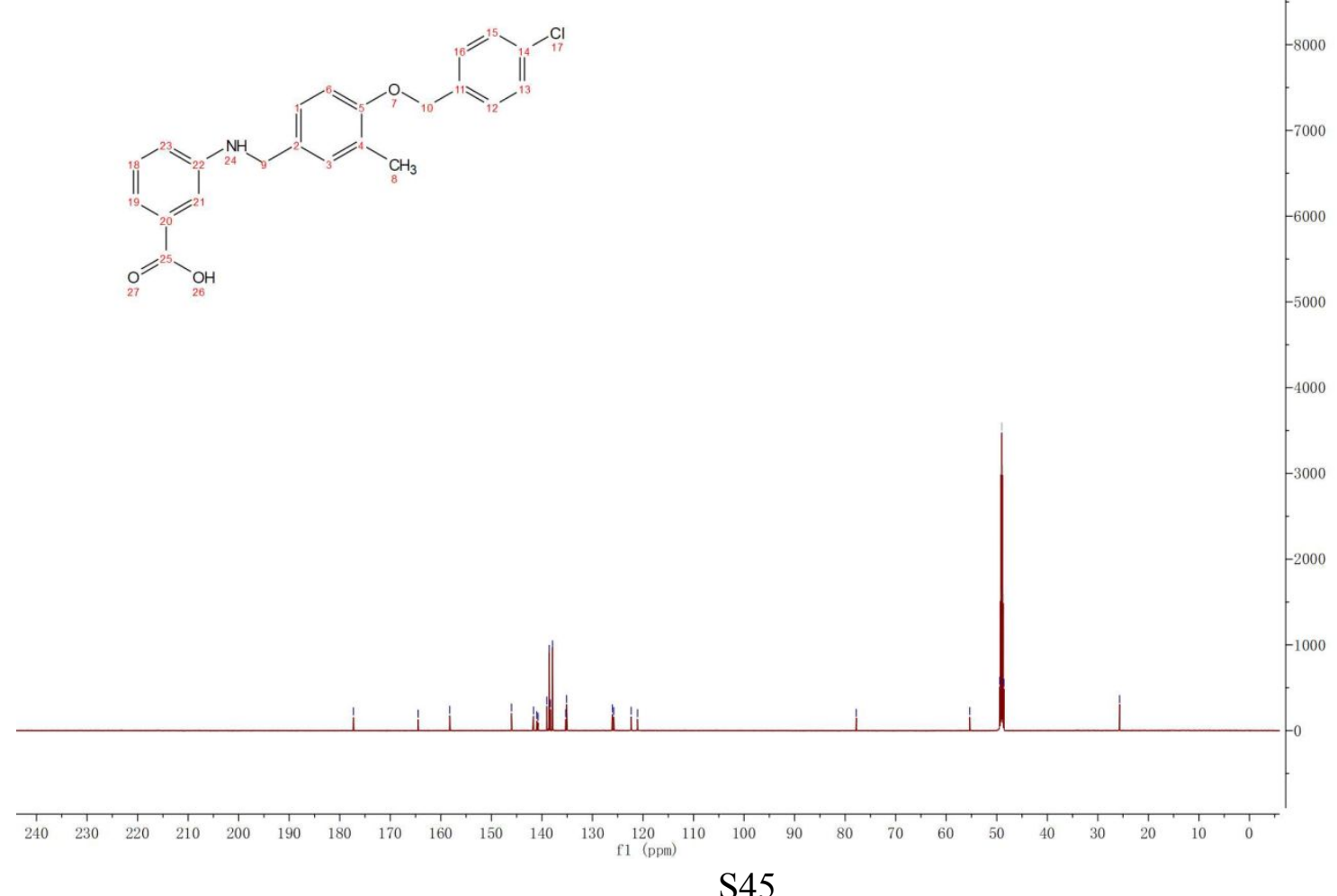

i

$-9000$

S45 
xd 7 51 600 PROTON 02

।

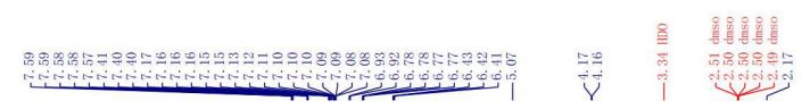<smiles></smiles><smiles>[Tl]</smiles>

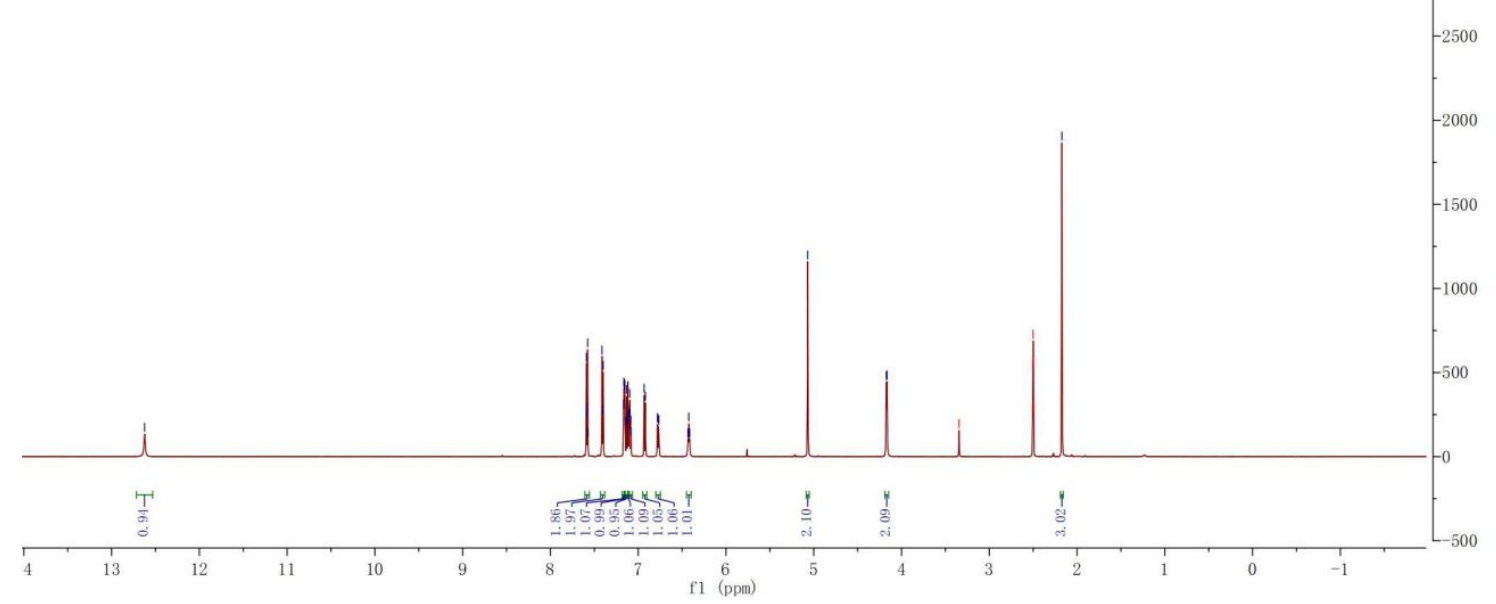

xd 7 751600 CARBON 01 xd $7 \_51600$

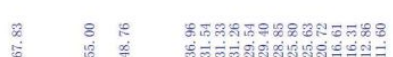

$\frac{1}{1} \quad \frac{1}{1} \frac{1}{1}$

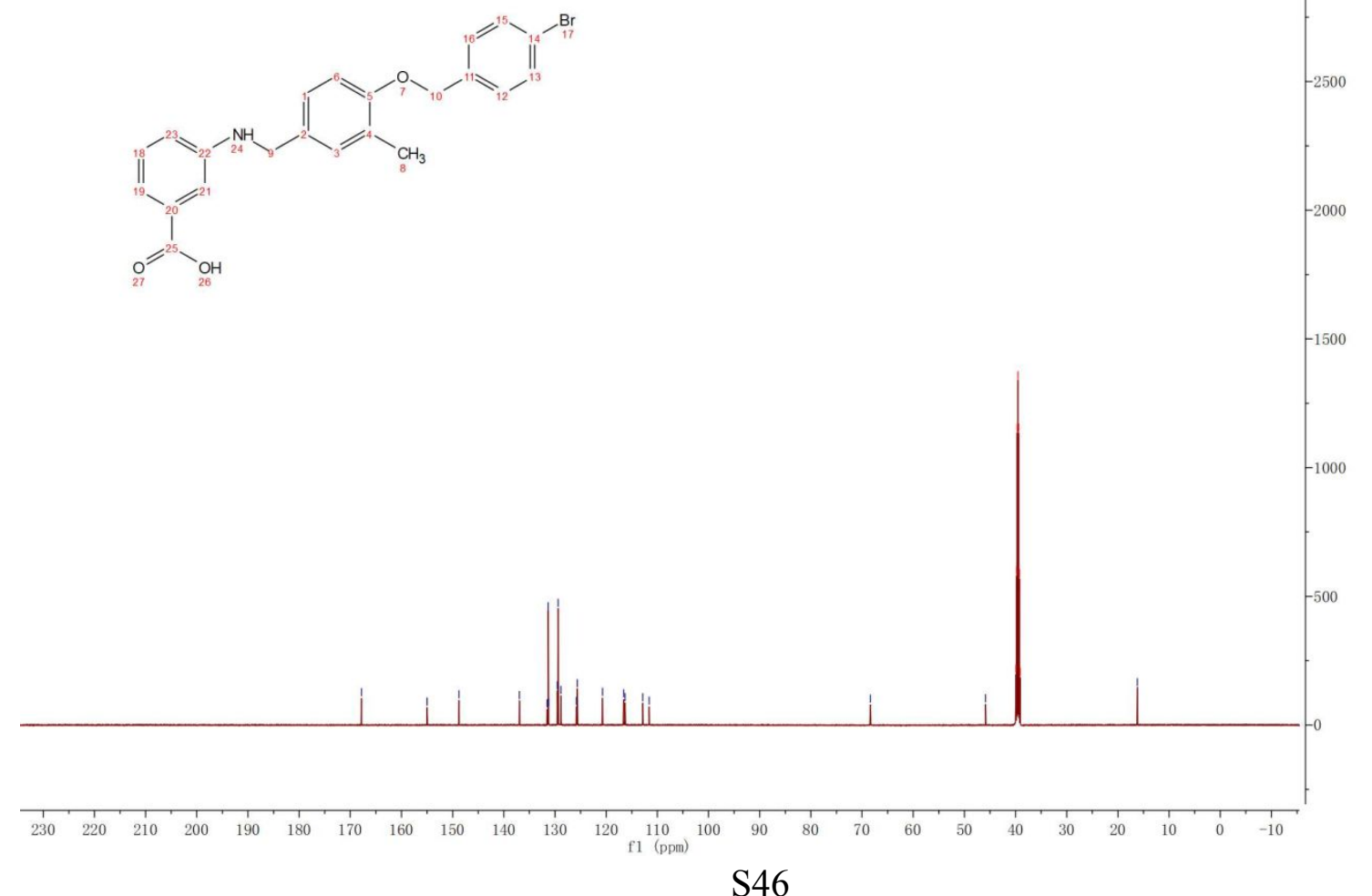

(n)

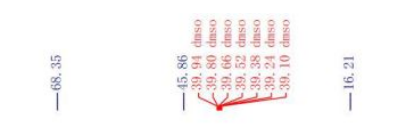

$-3000$ 
<smiles></smiles>

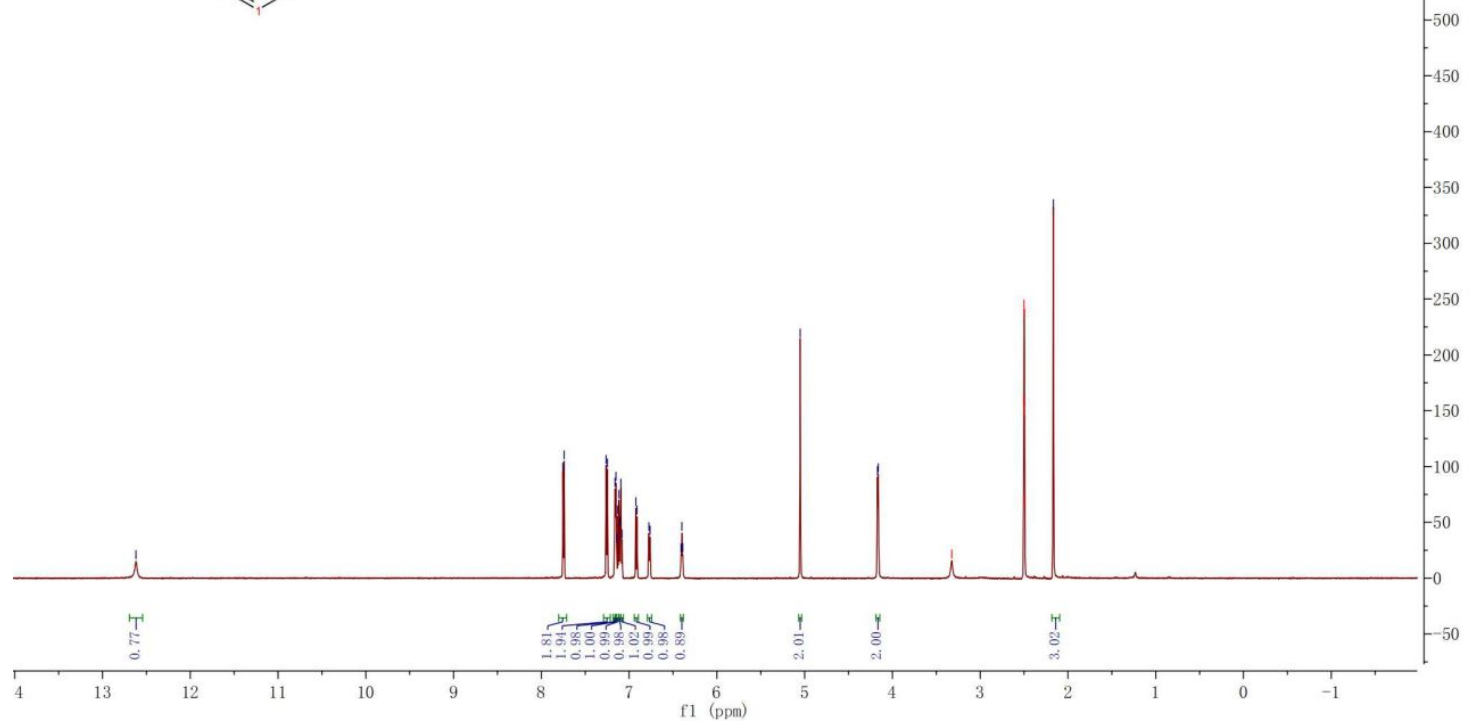

xd 7 753 600 CARBON_01

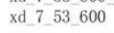
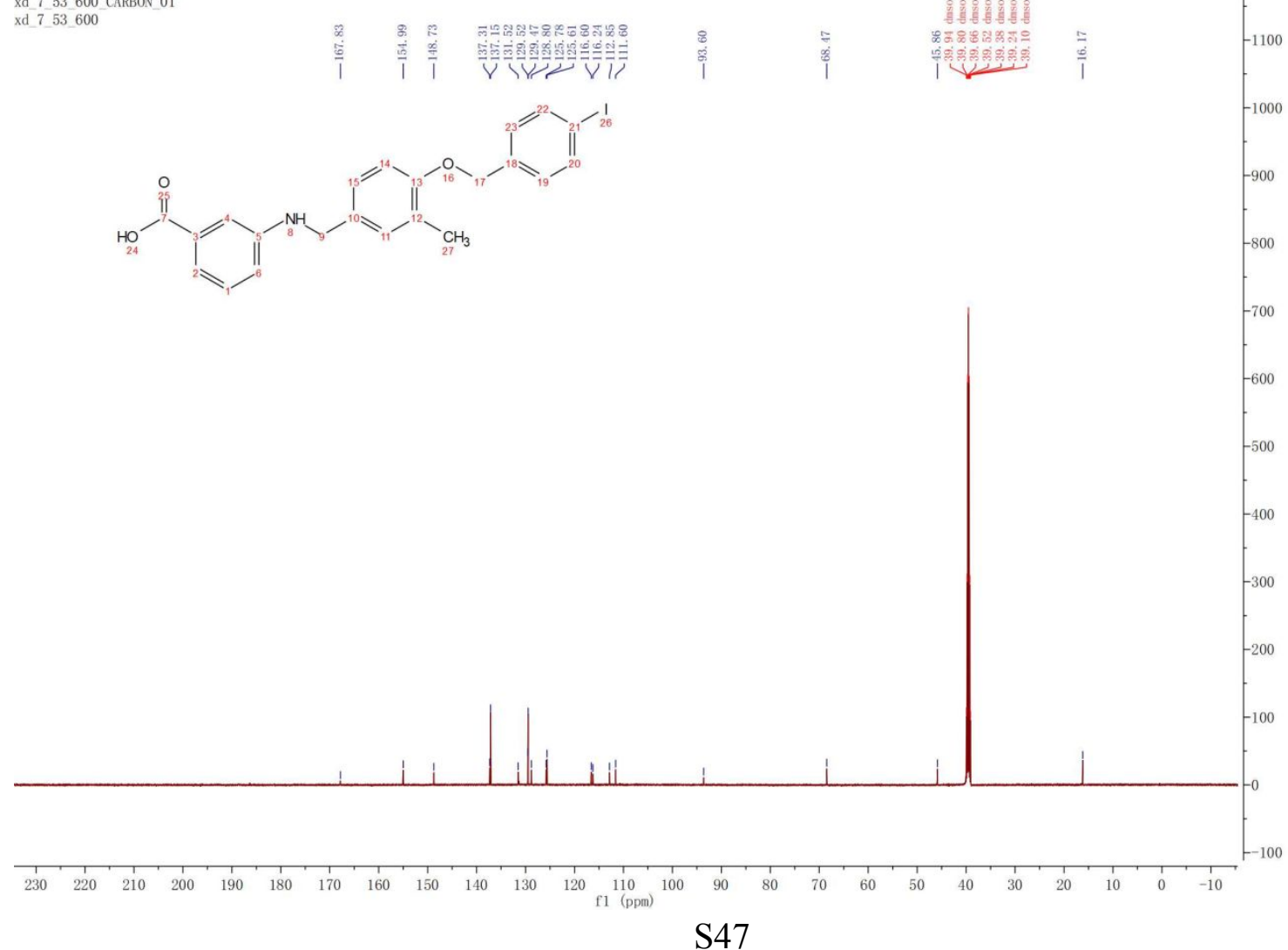
23

xd 6 - 193 PROTON 02 $\vec{b}$
$\stackrel{0}{1}$
$\stackrel{1}{1}$

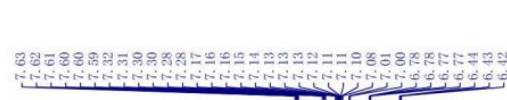

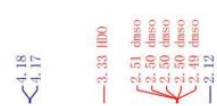

$-11000$

(19)

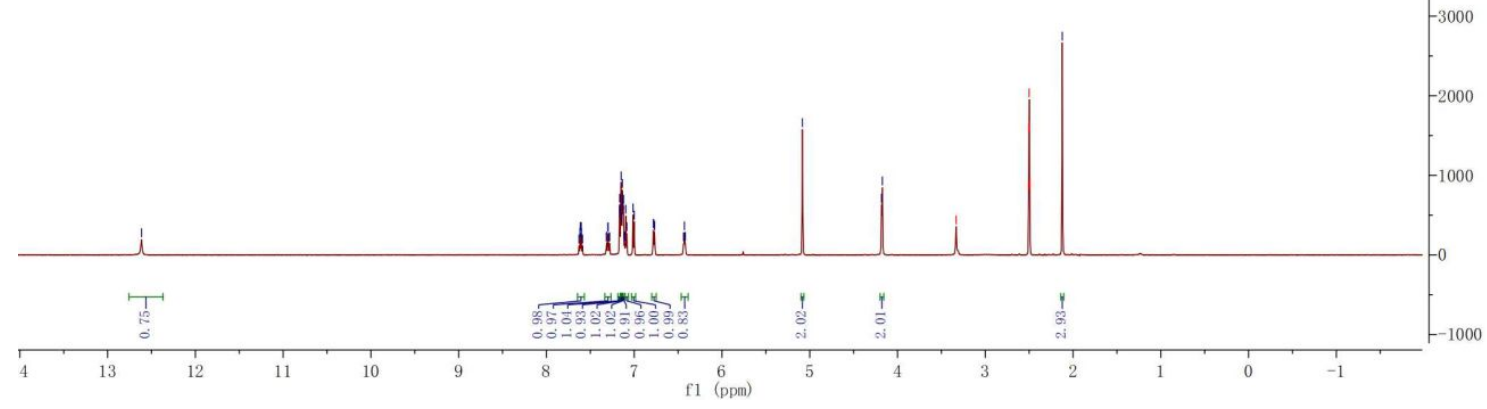

xd 6 6 193 CARBON 01 xd $6 \_193$

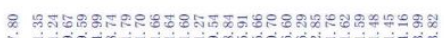

I V)
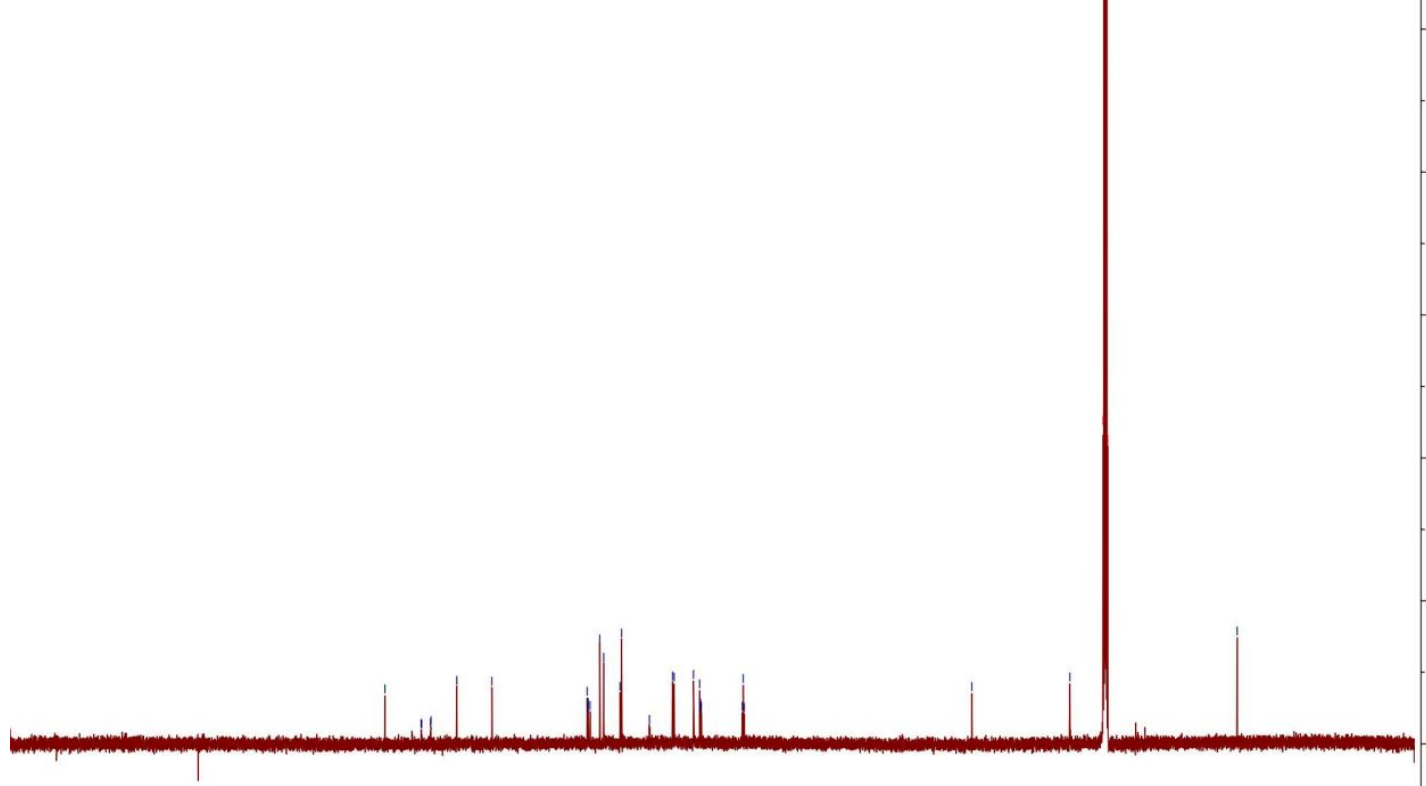

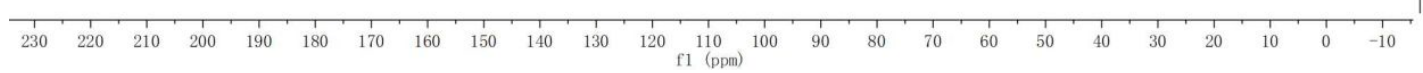



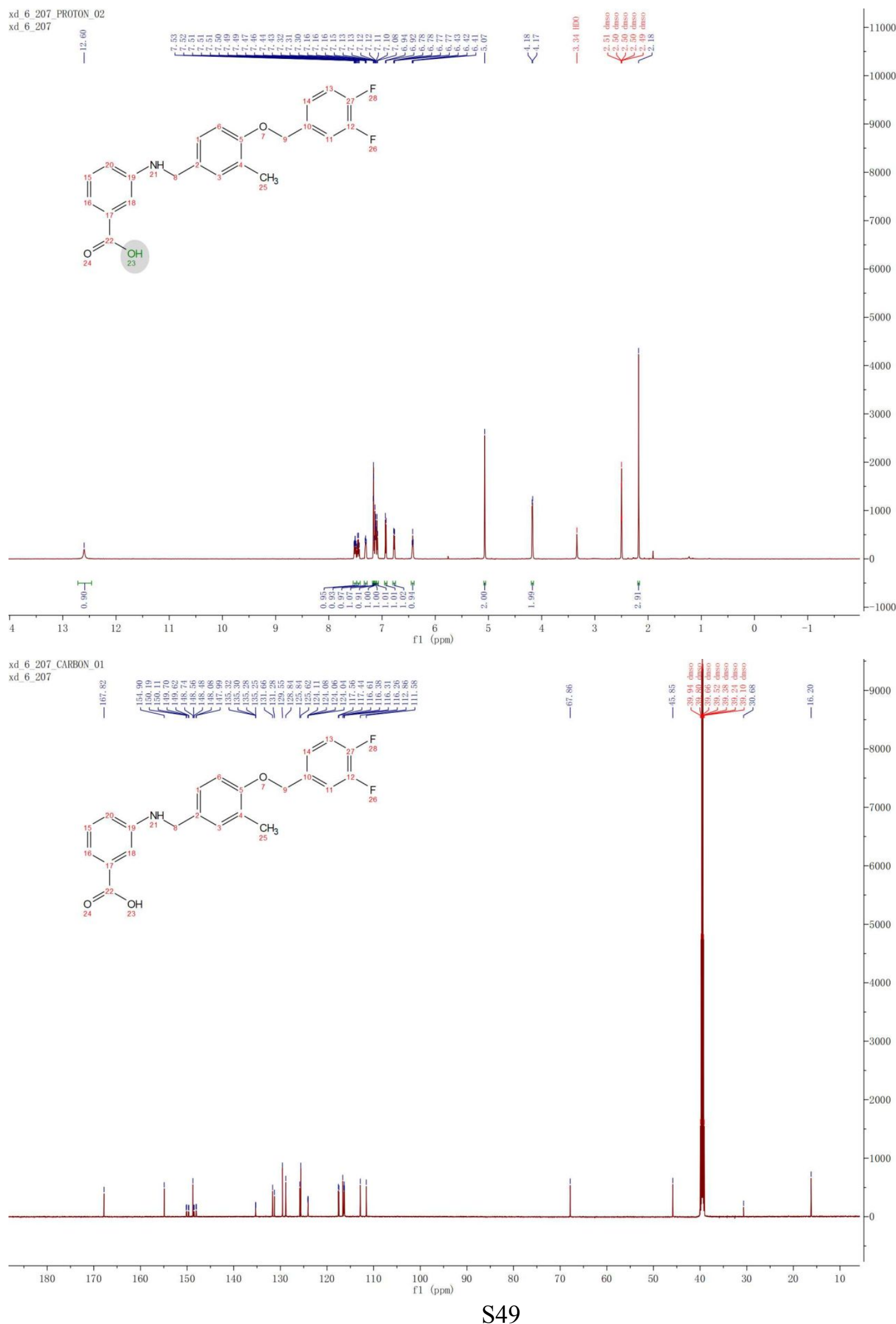
xd $6 \quad 215600$ PROTON 04

xd $6 \_215-600$
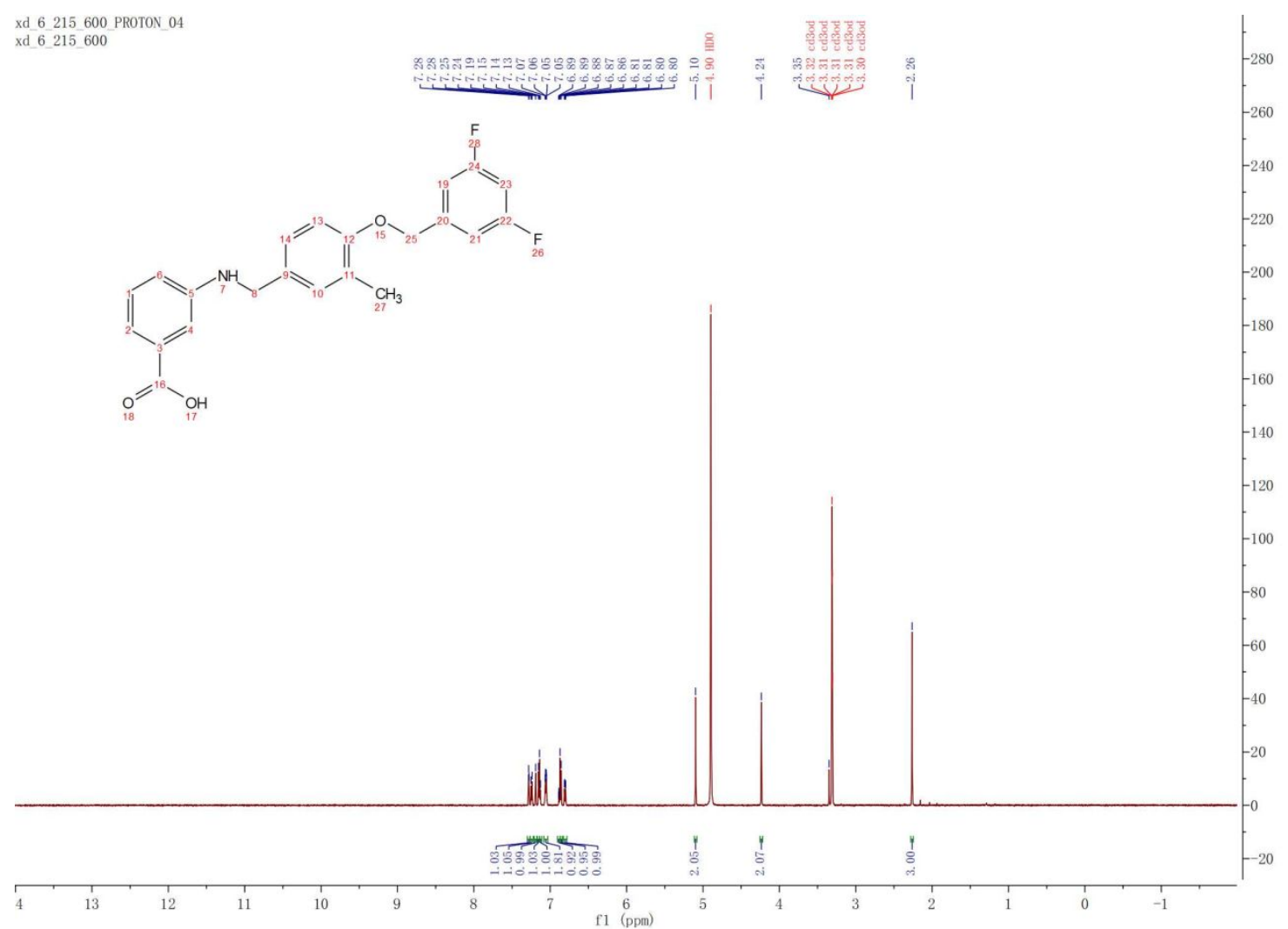

xd 6 215 600 CARBON 01

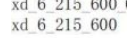
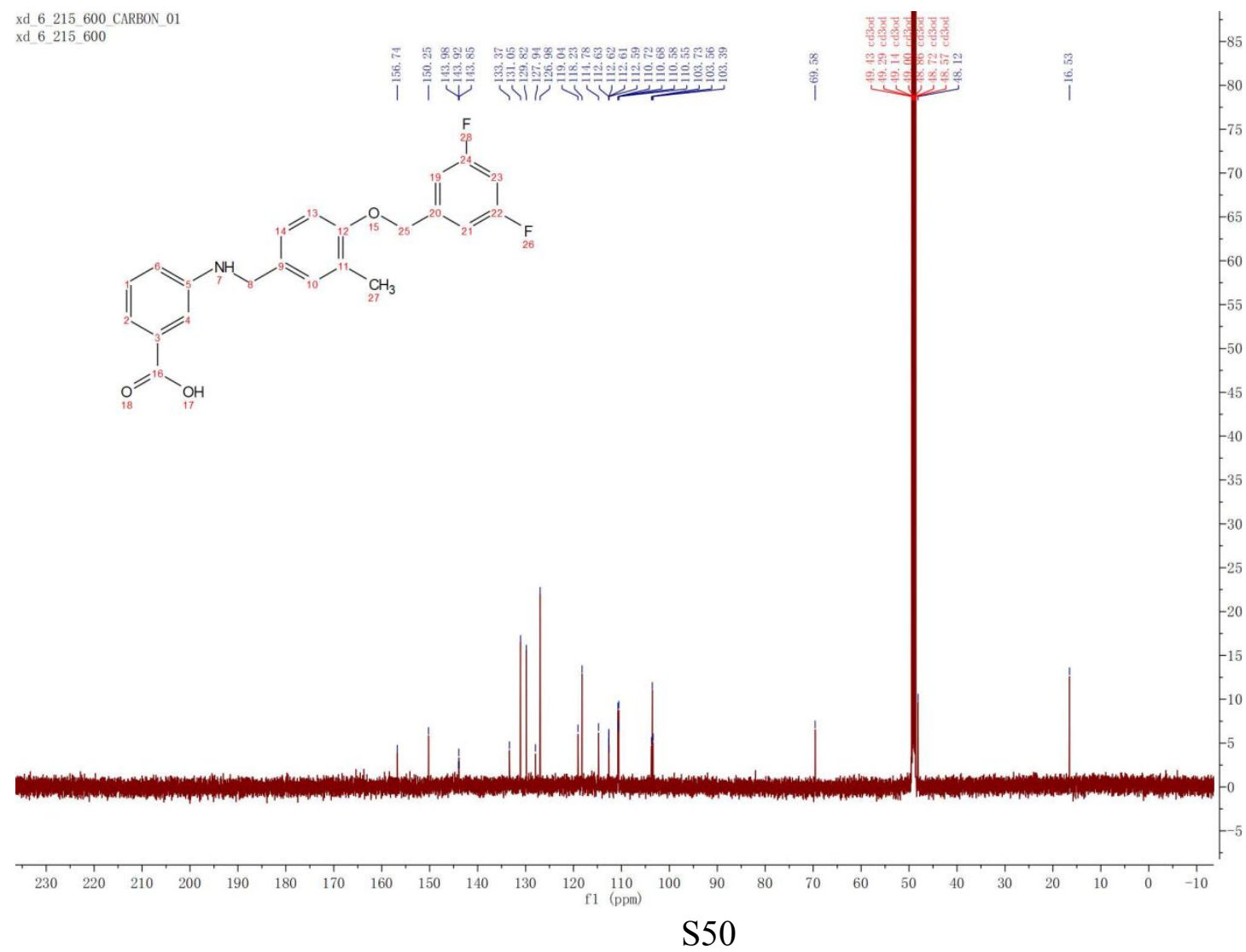
xd 6 253 PROTON 02

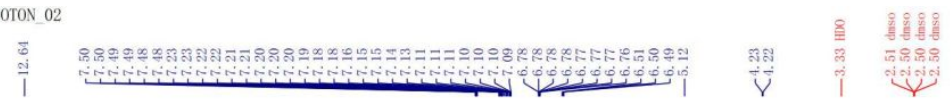<smiles></smiles>

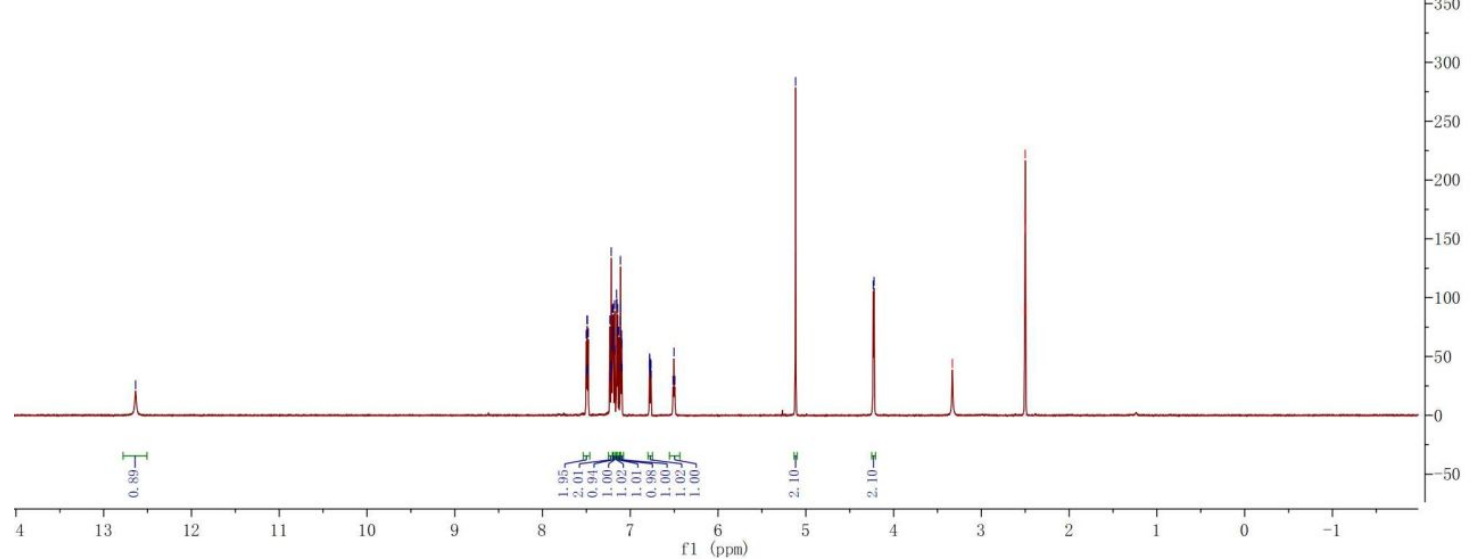

xd 6 253 CARBON 01 xd $6 \_253$
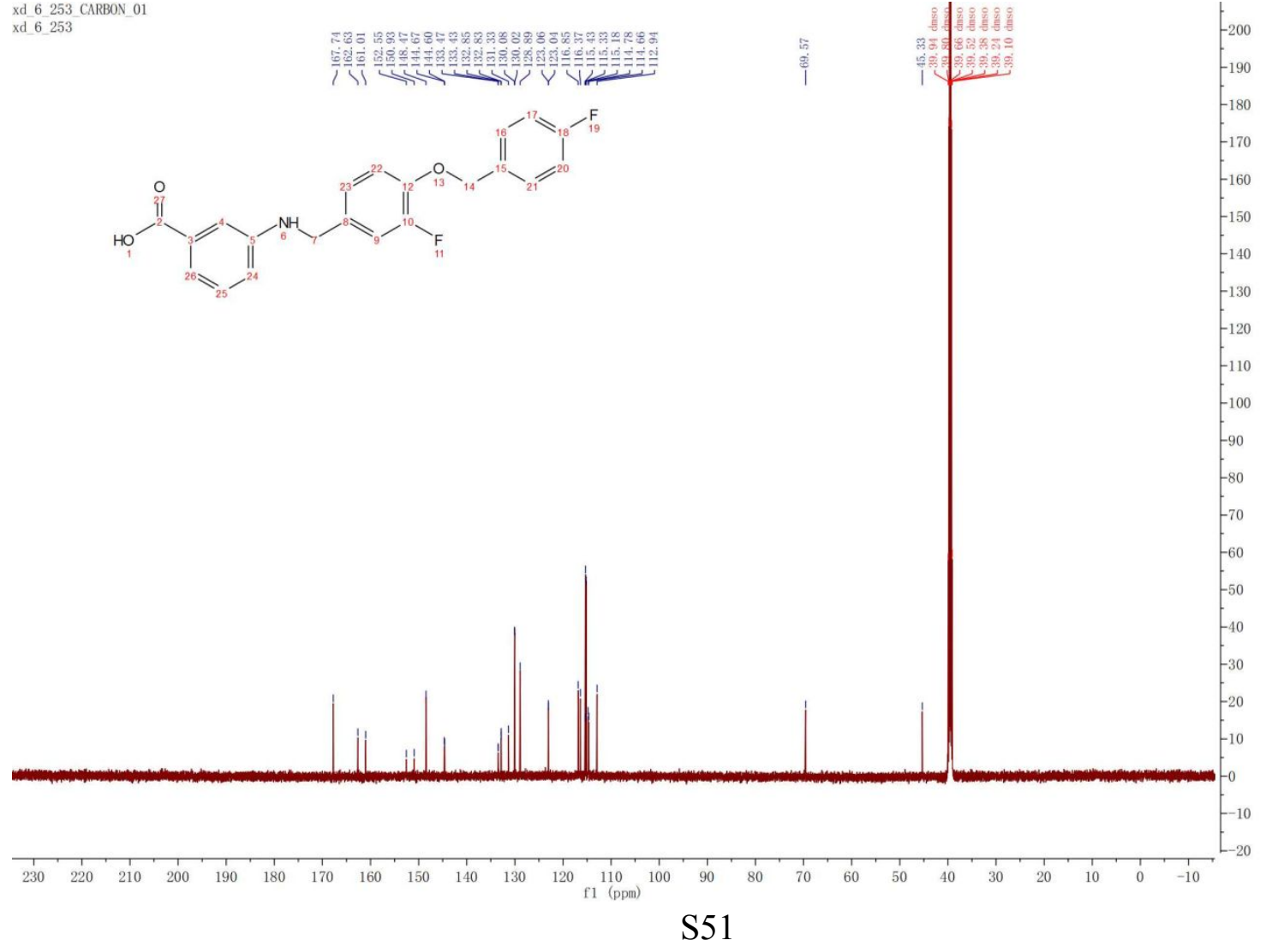
xd 6 291 600 PROTON 02

xd 6_291 600

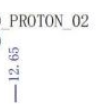<smiles></smiles>

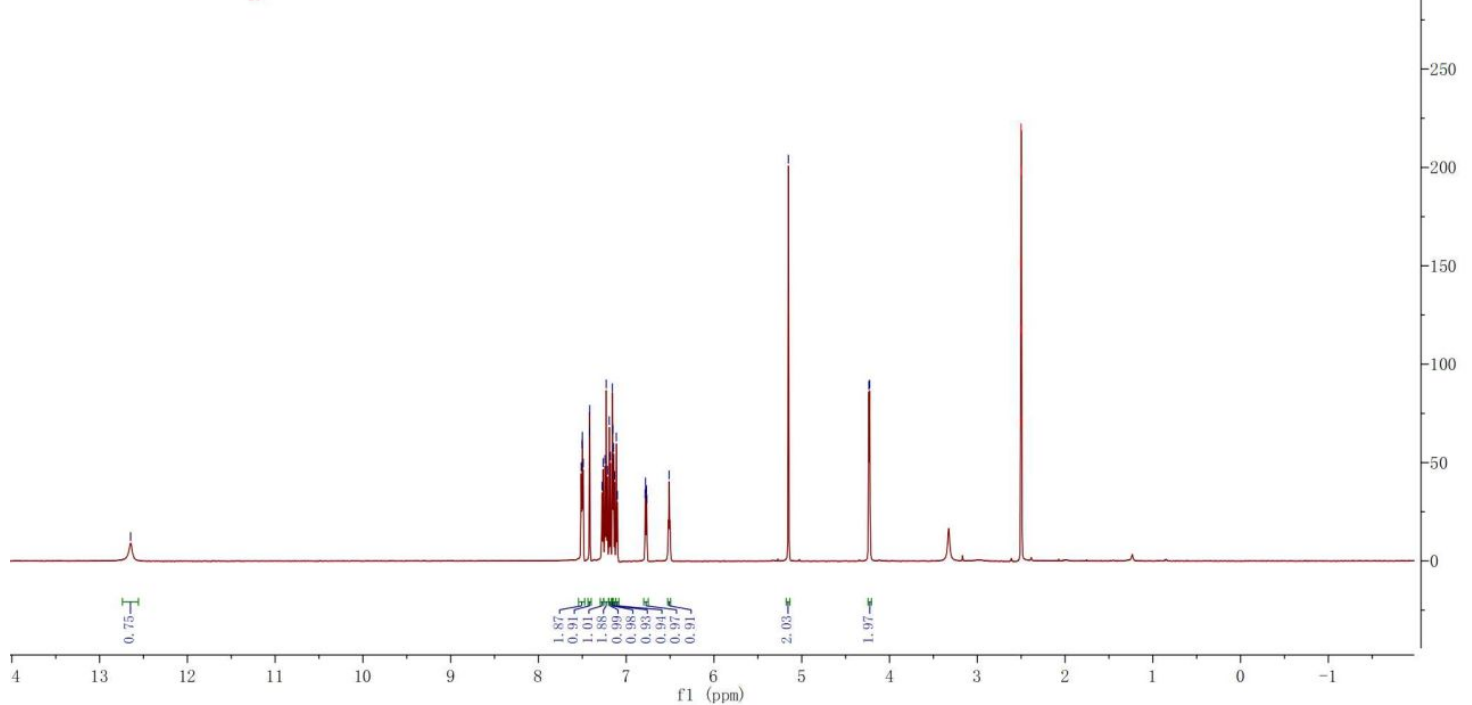

xd 6 291 600 CARBON 01 xd 6291600
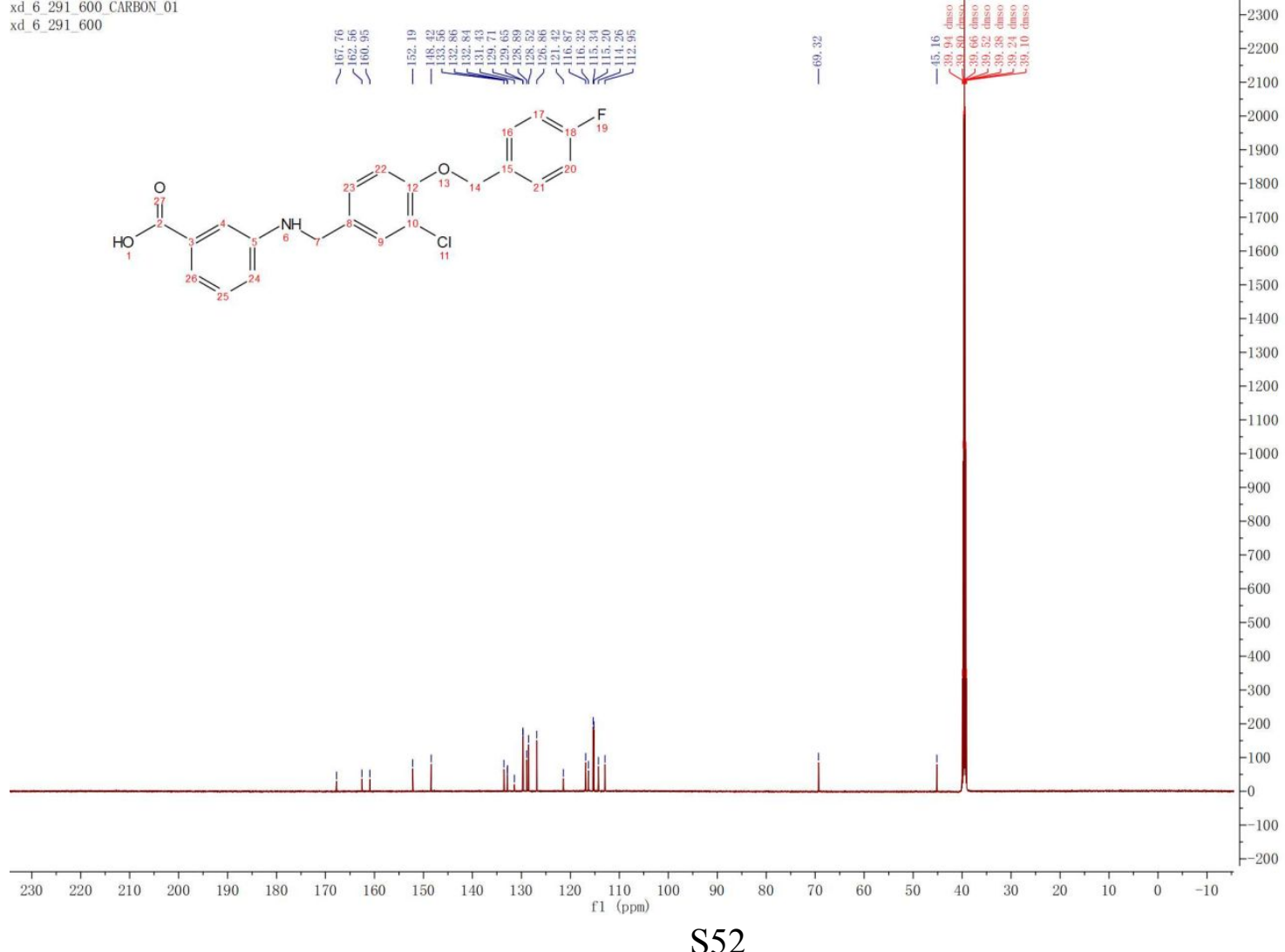


\section{8}

$\begin{array}{lll}\text { xd } 6 & 249 \text { PROTON } 02 \\ \text { xd } 6 & 249\end{array}$

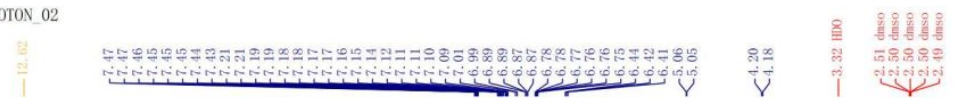

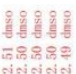

$\sqrt[4]{3.0899}$<smiles></smiles>

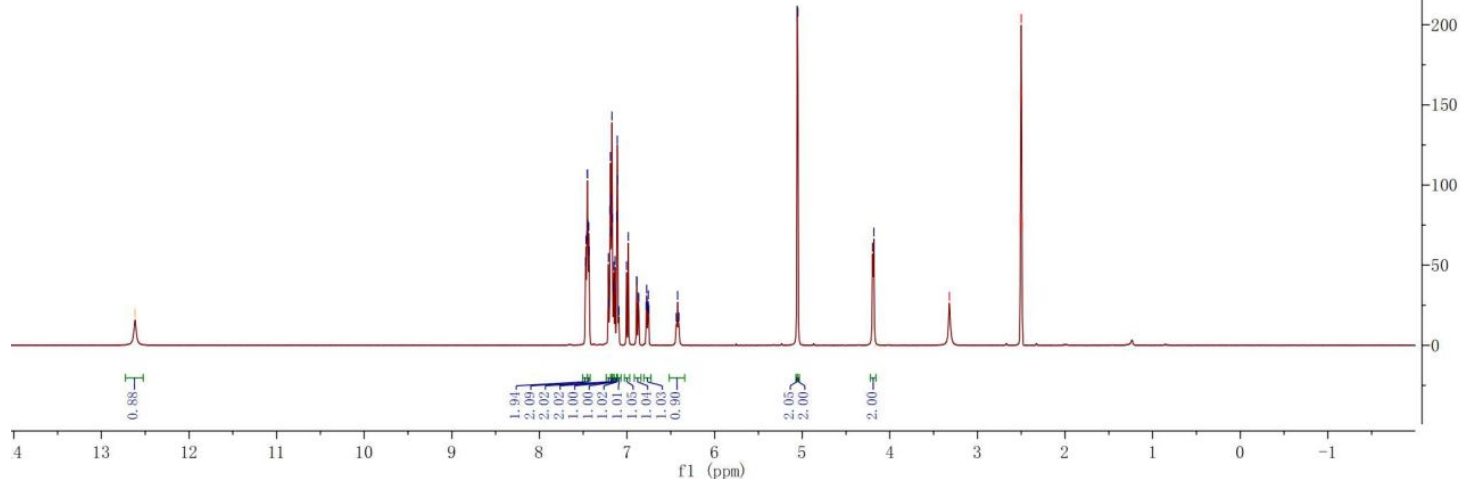

xd 6_249_CARBON 01 $\begin{array}{llll}x d & 6 & 249 \\ x d \quad 6 & 249\end{array}$

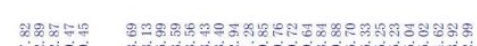
Y ᄂ<smiles>O=C(O)c1cc(NCc2ccc(OPc3cnc(F)nc3)c(OPc3cncc(F)n3)c2)ncn1</smiles>

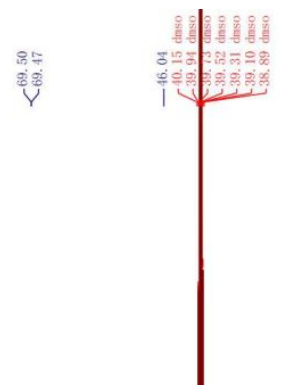

-80
-75
-65
-60
-50
-40
-30
-30
-25
-10
-20

$$
\frac{1}{230}
$$
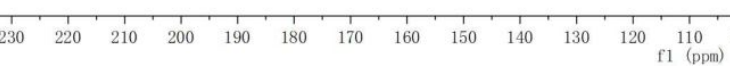

S53 
xd 7 75 600 PROTON 02

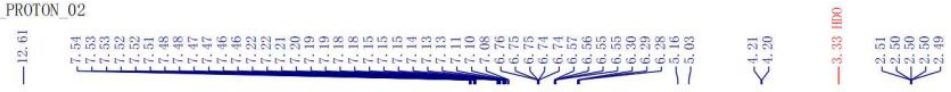<smiles></smiles>

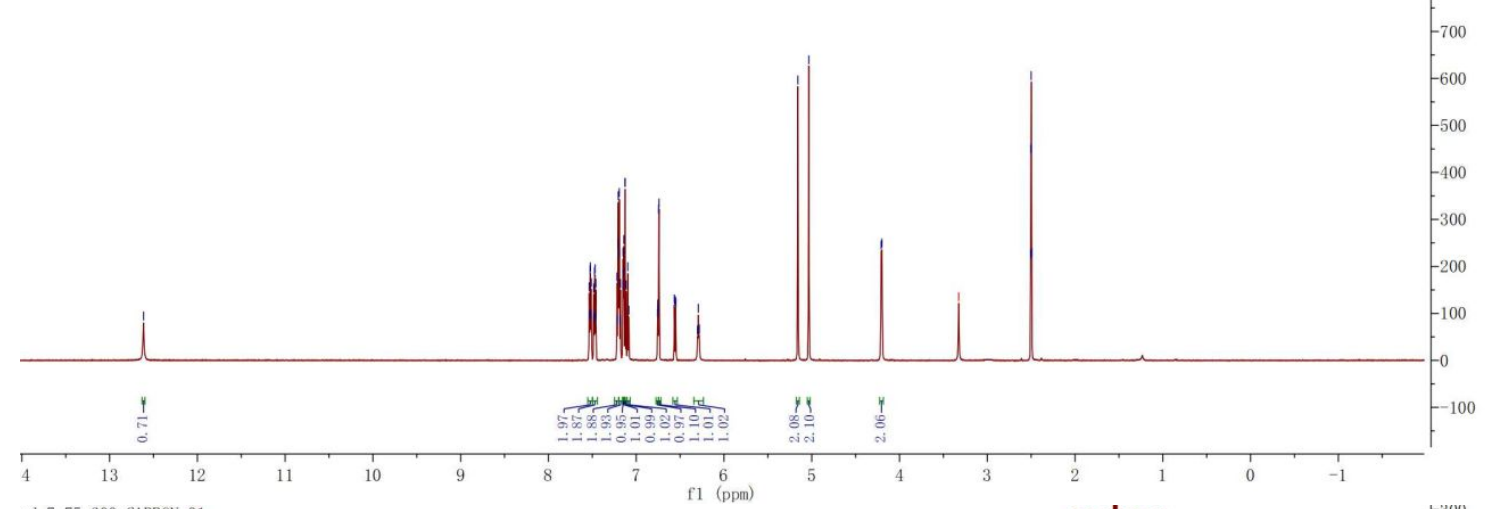

xd 7 7 $75 \_600$ CARBON 01 xd $775 \_600$

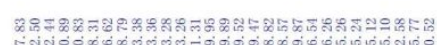
1<smiles></smiles>
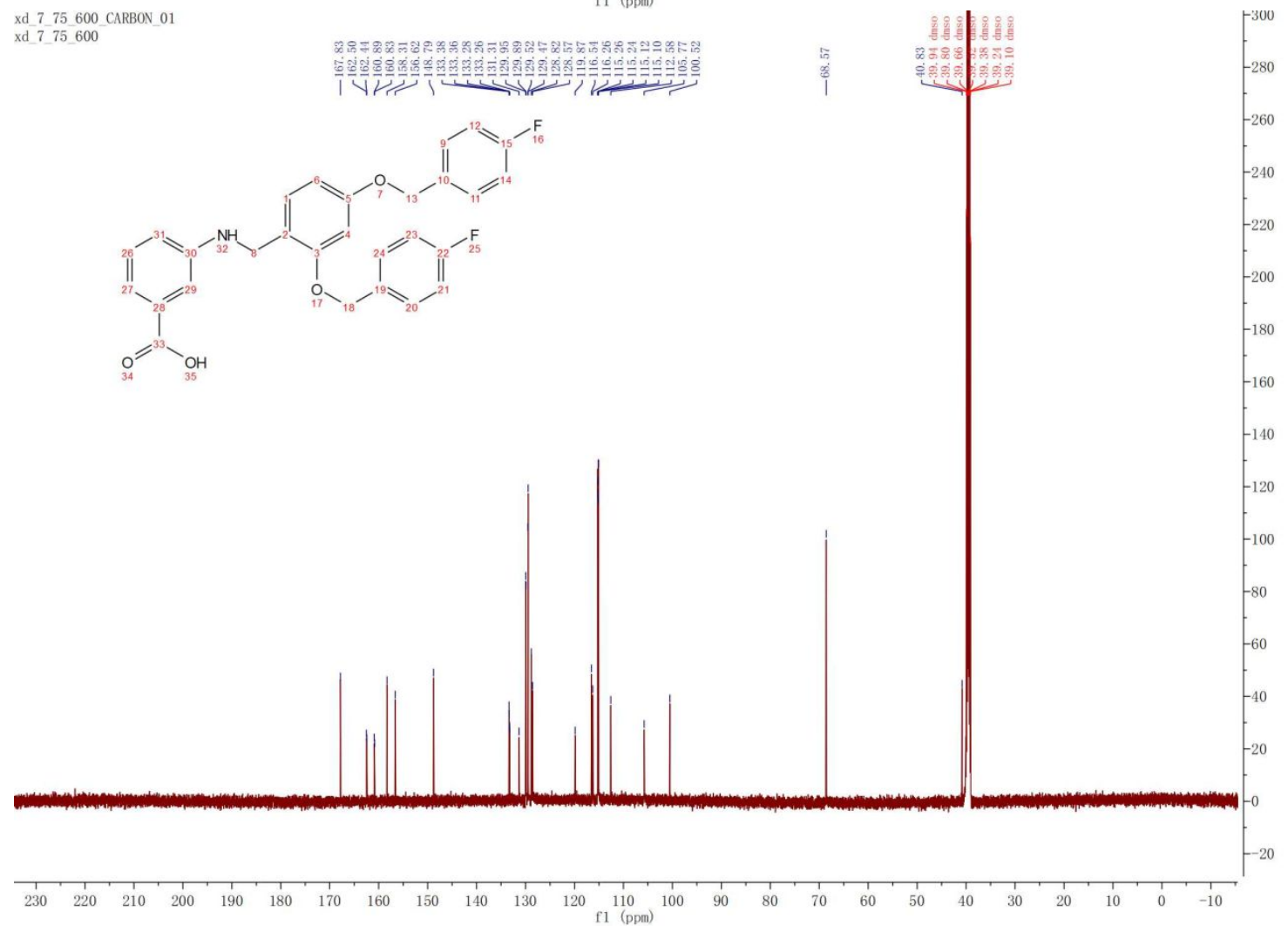
30

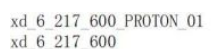

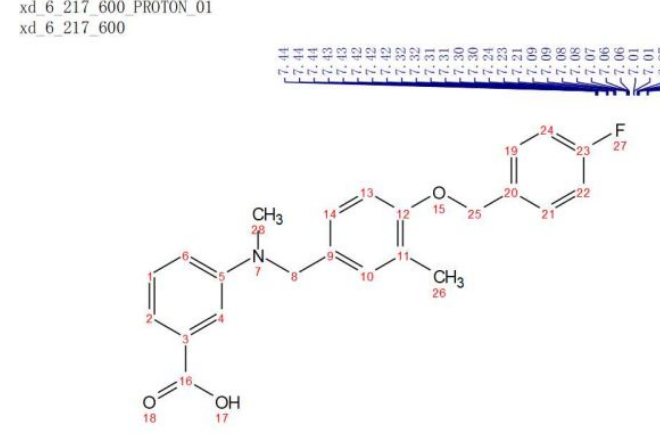

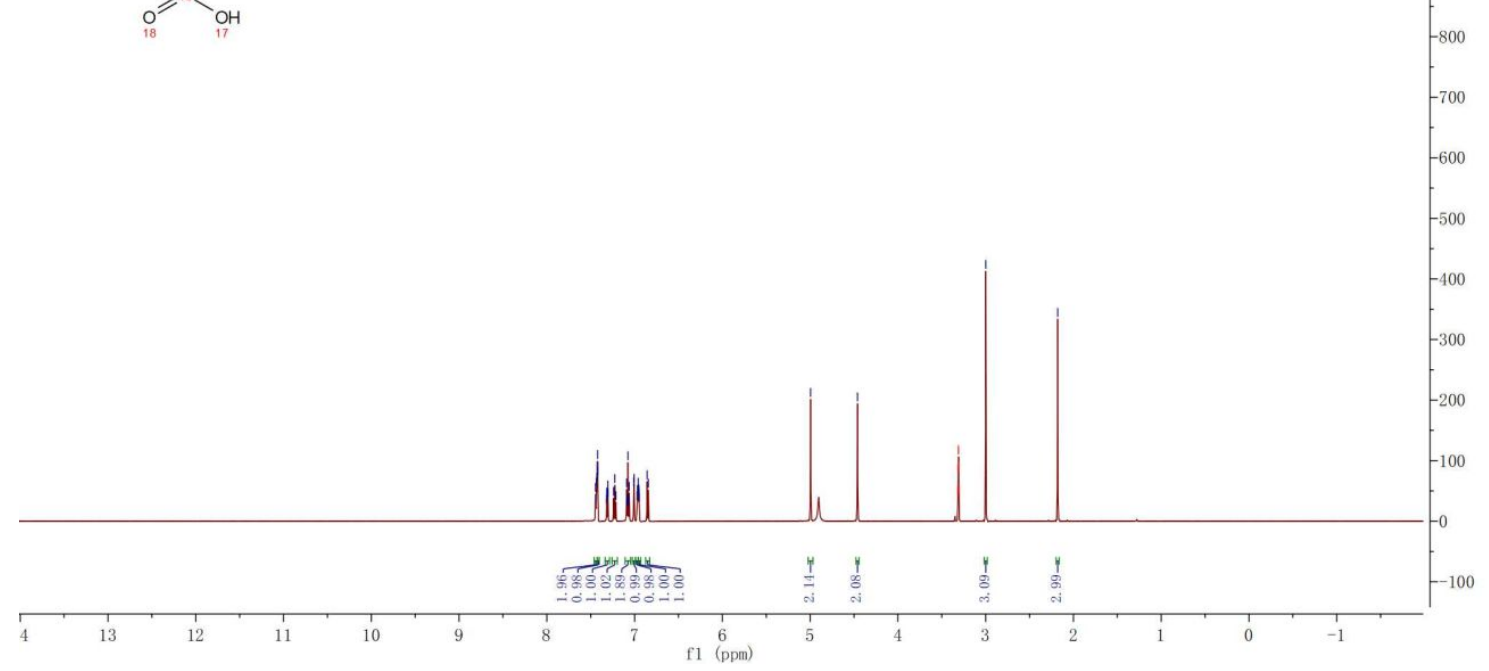

xd 6 217 600 CARBON 01 xd $6 \_217 \_600$

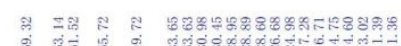

।<smiles></smiles>

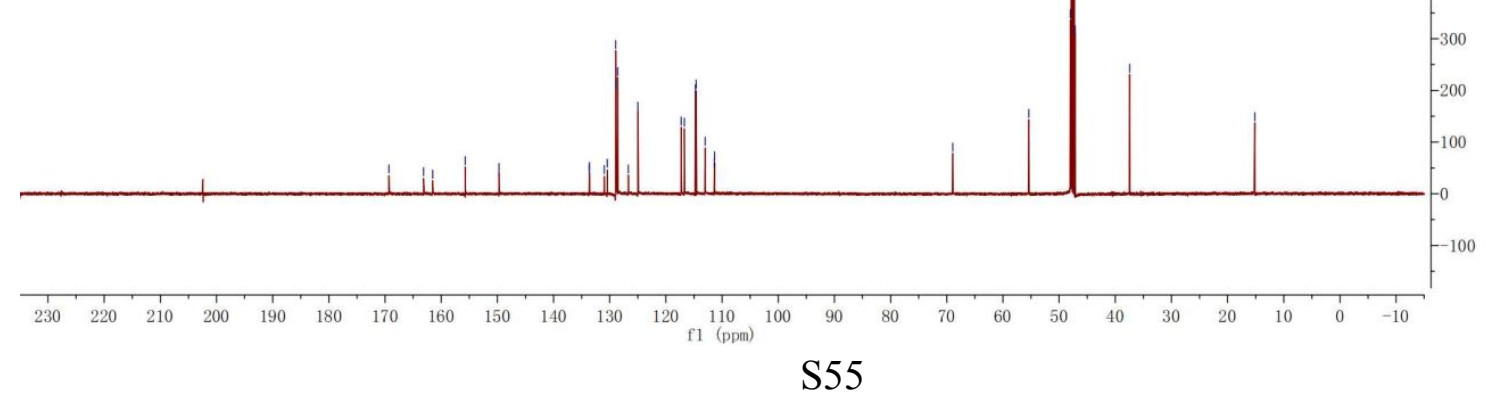


31
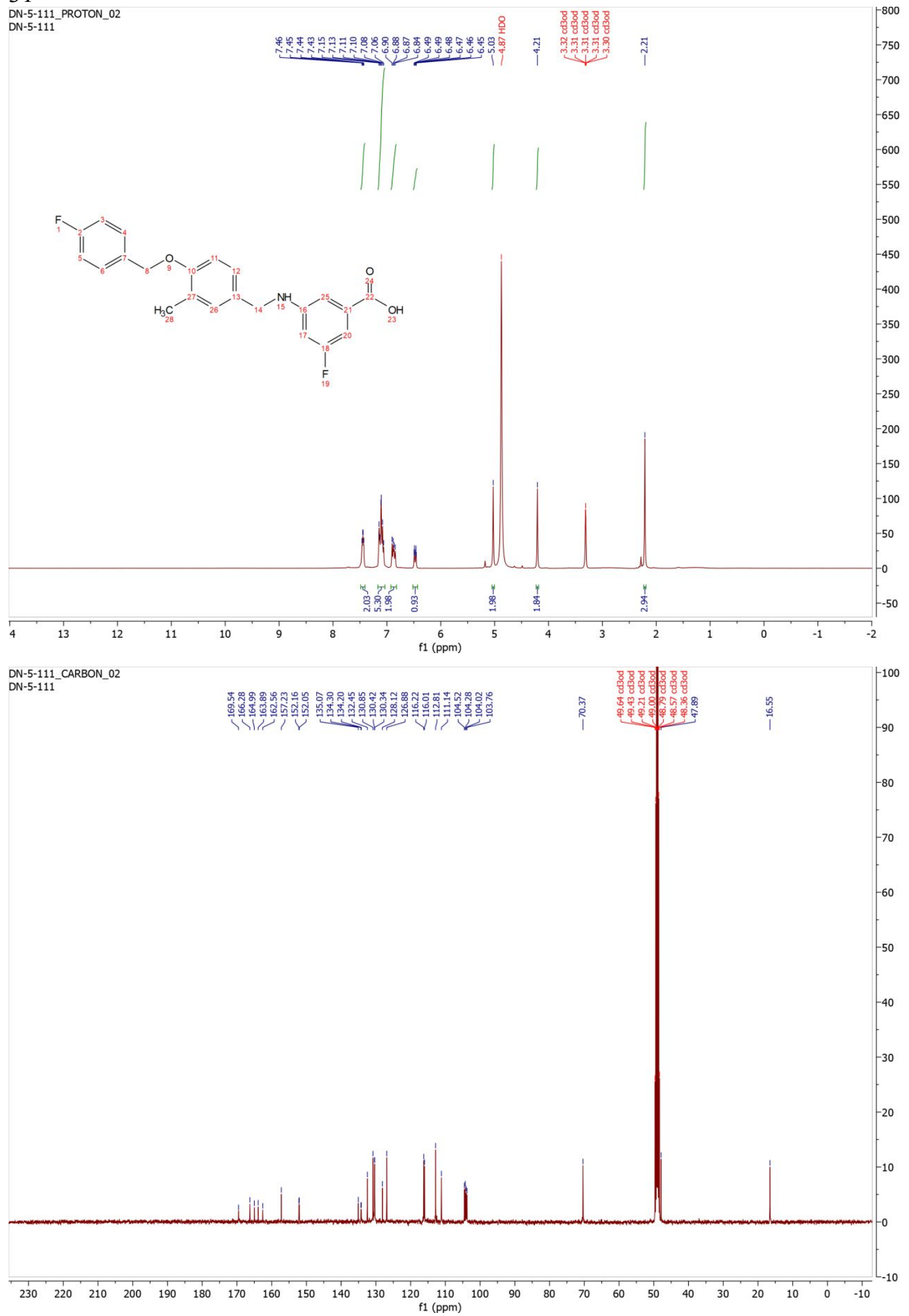

S56 
32
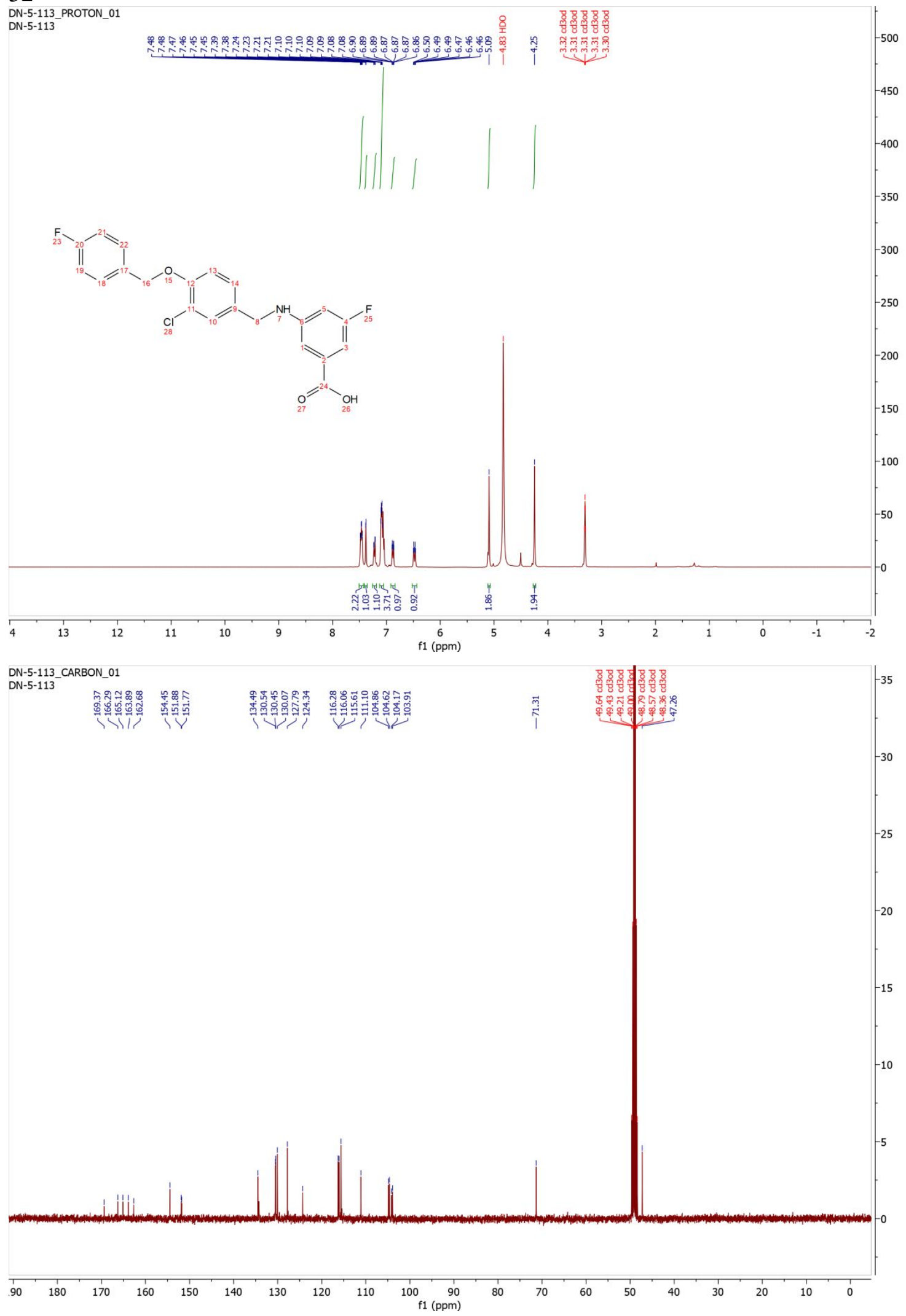

S57 
33

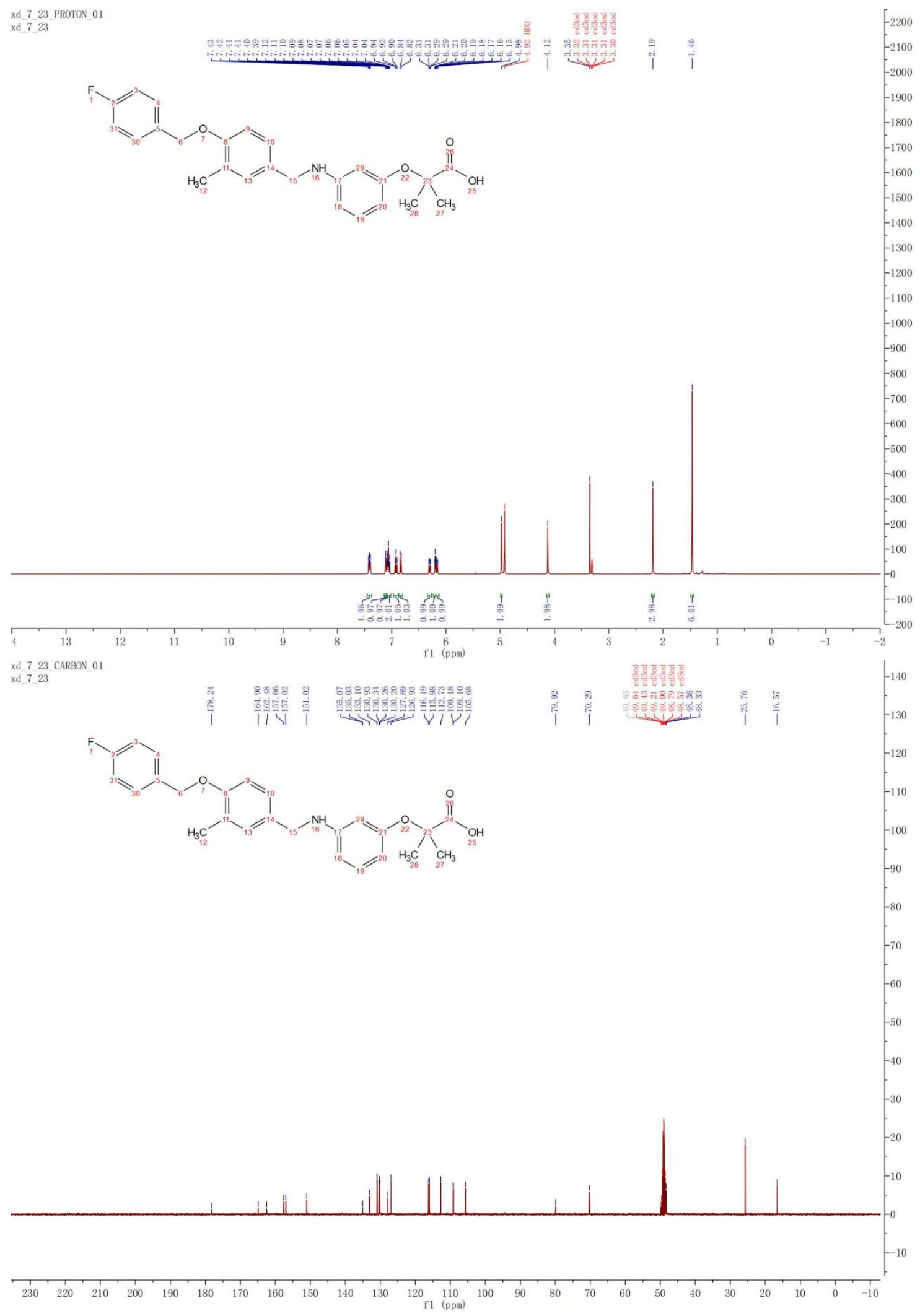

S58 


\section{HPLC Traces}

\begin{tabular}{|c|c|c|c|c|c|c|c|c|c|}
\hline Peak & t & RT & Area $\boldsymbol{t}$ & Height $\nrightarrow$ & Type $\nrightarrow$ & Saturated $\boldsymbol{\emptyset}$ & Width $\mapsto$ & FWHM $九$ & SNR $\oplus$ \\
\hline & 1 & 7.2 & 7611.9 & \begin{tabular}{|l|}
1917.3 \\
\end{tabular} & & & 0.293 & 0.059 & \\
\hline & 2 & 8.427 & 94.93 & 23.43 & & & 0.24 & 0.057 & \\
\hline
\end{tabular}

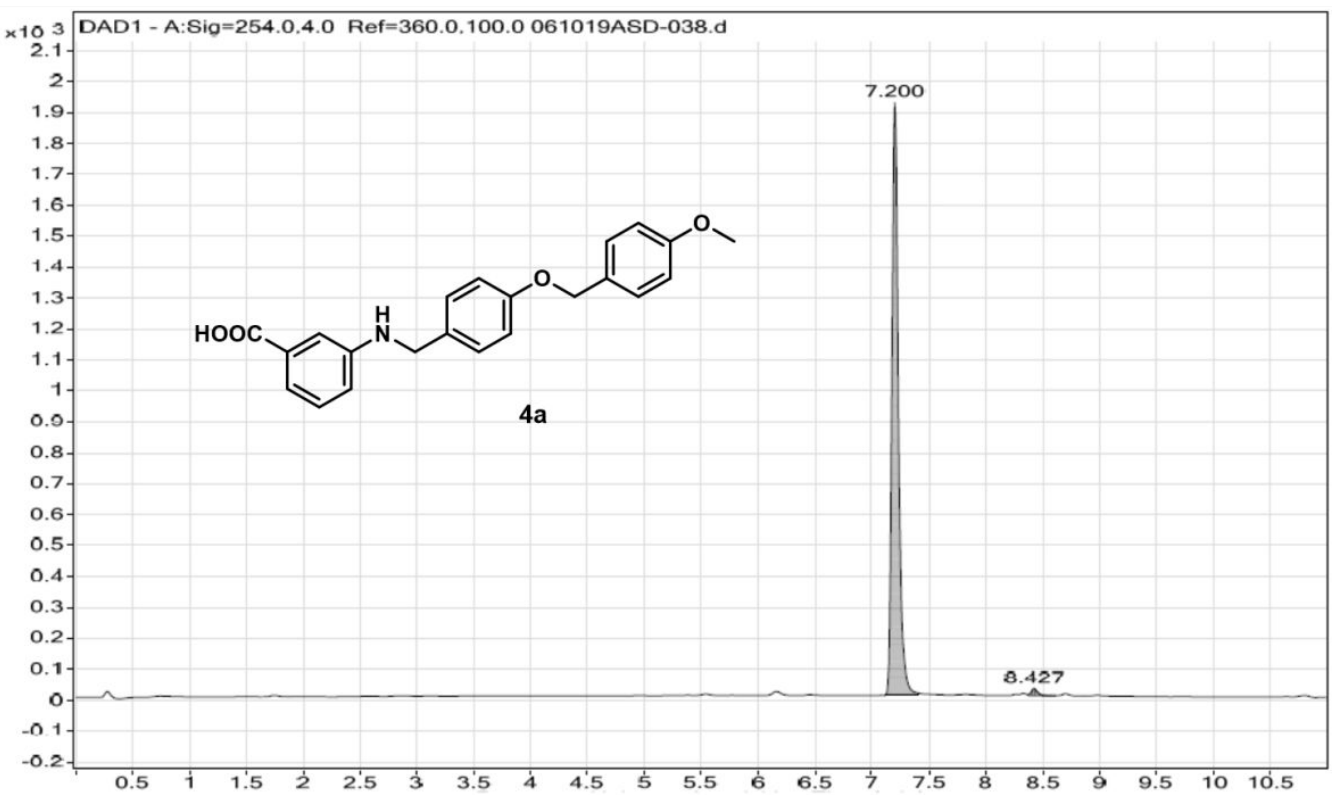

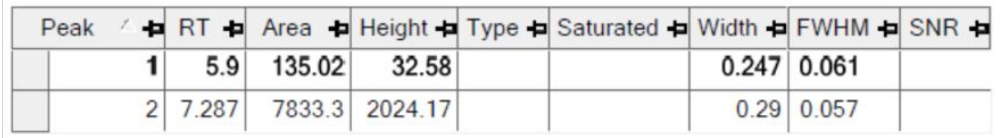

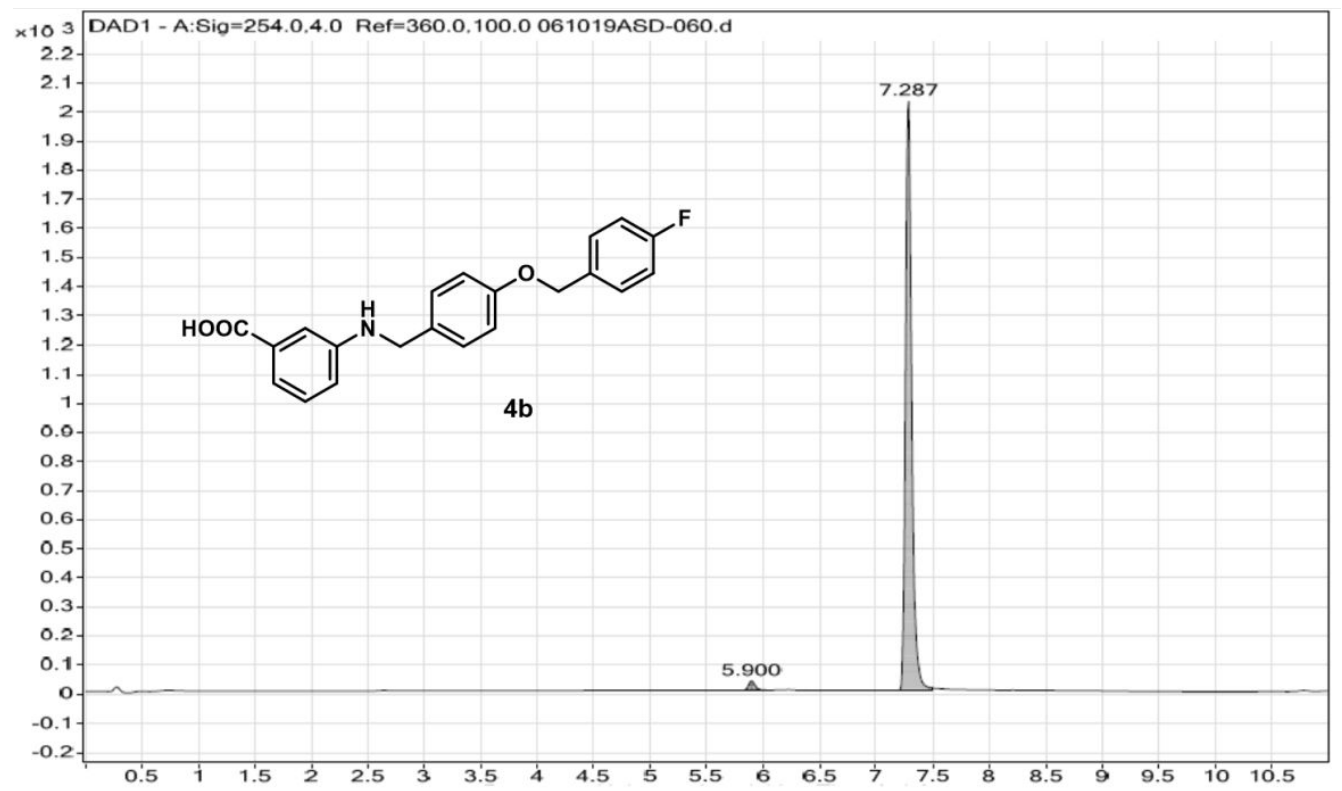




\begin{tabular}{|c|c|c|c|c|c|c|c|c|}
\hline Peak & $\mapsto$ & $R T \mapsto$ & Area $\mapsto$ & a Height $九$ & Type $わ$ Saturated $ゃ$ & Width & FWHM ↔ & SNR \\
\hline & 1 & 7.853 & 7214.4 & 1803.97 & & 0.3 & 0.059 & \\
\hline
\end{tabular}

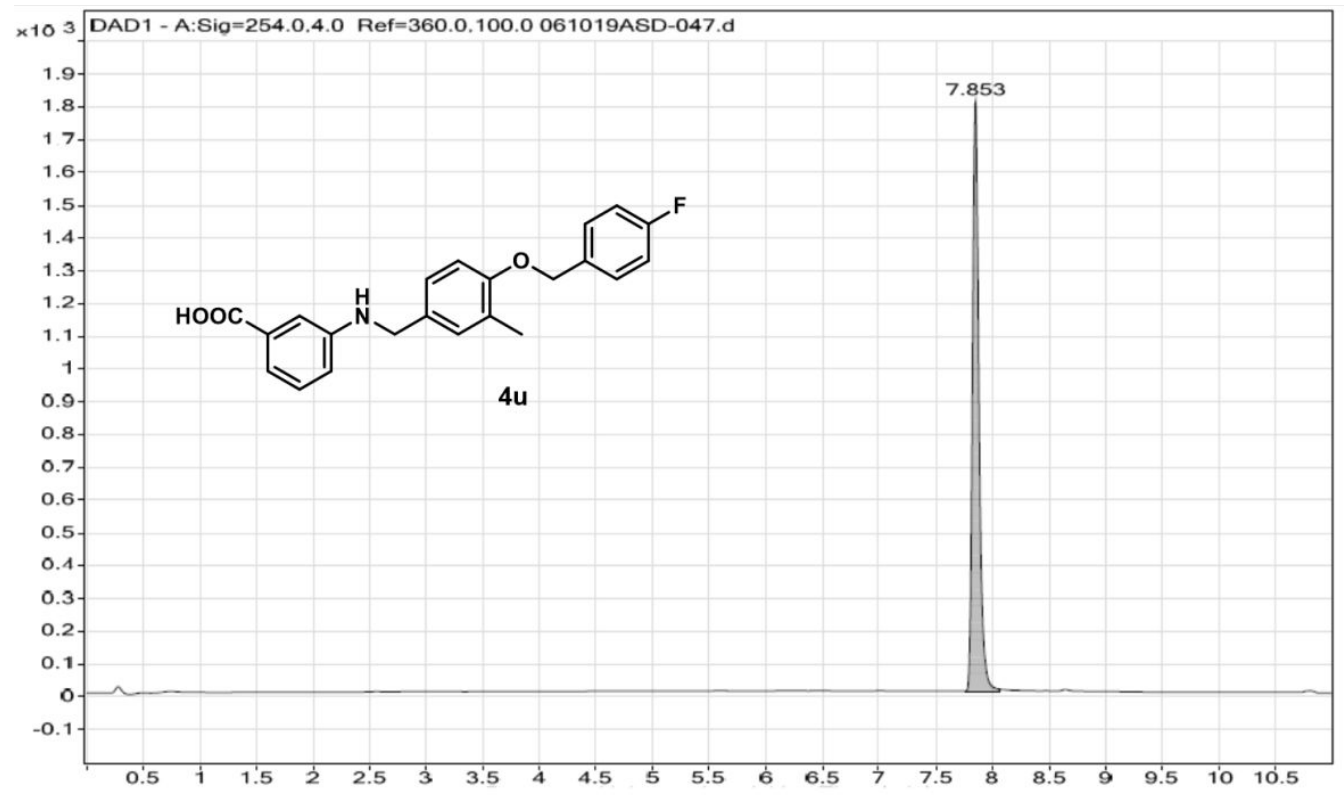

\begin{tabular}{|c|c|c|c|c|c|c|c|c|c|}
\hline Peak & t & RT & Area わ & Height $\nrightarrow$ & Type わ & Saturated $\nrightarrow$ & Width & FWHM & 口 SNR 中 \\
\hline & 1 & 7.913 & 7258.6 & 1920.86 & & & 0.287 & 0.056 & \\
\hline
\end{tabular}

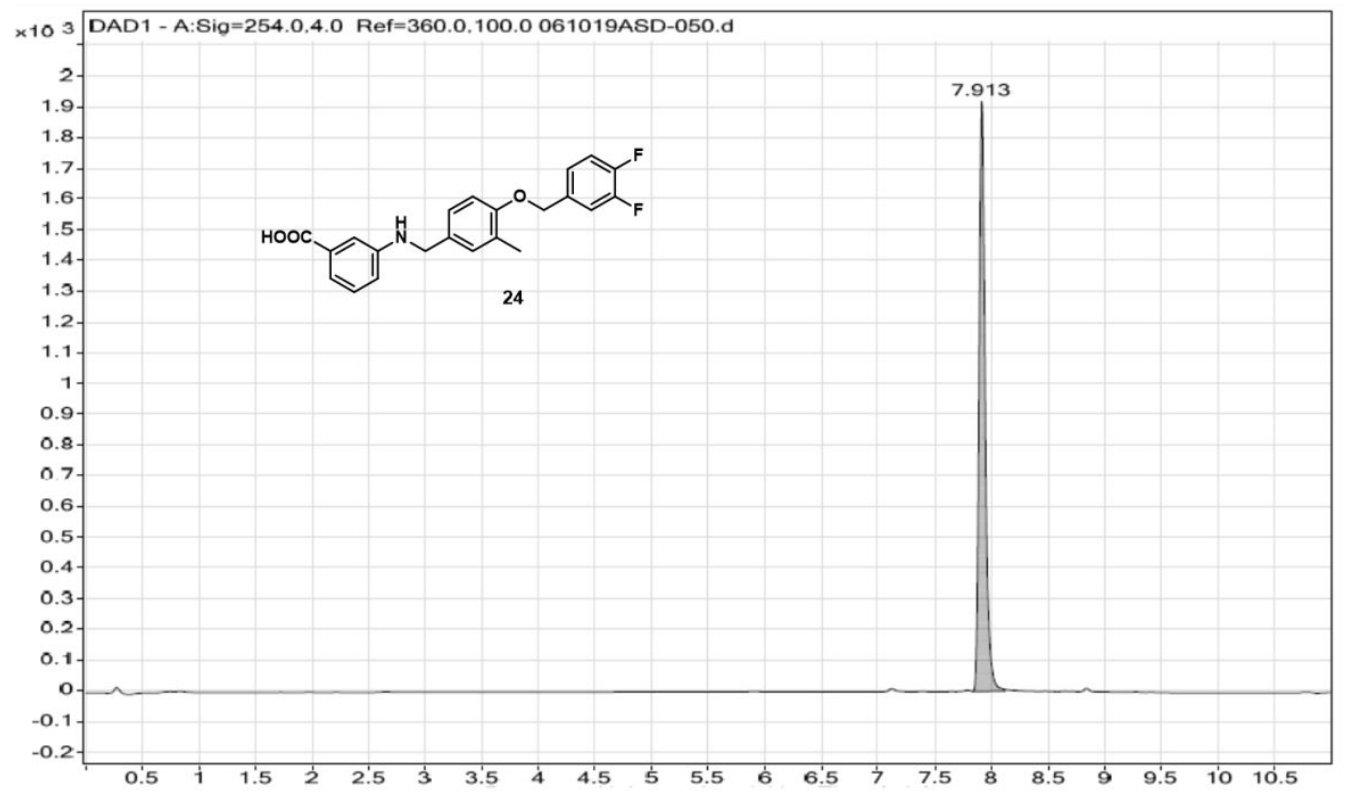




\begin{tabular}{|c|c|c|c|c|c|c|c|c|c|}
\hline Peak & t & RT $\mapsto$ & Area $\downarrow$ & Height $九$ & Type $九$ & Saturated & Width & - $\mathrm{FWHM}$ † & SNR † \\
\hline & 1 & 0.28 & 115.51 & 32.09 & & & 0.189 & 0.054 & \\
\hline & 2 & 3.867 & 62.05 & 16.78 & & & 0.193 & 0.052 & \\
\hline & 3 & 4.4 & 2570.6 & 805.61 & & & 0.226 & 0.048 & \\
\hline
\end{tabular}

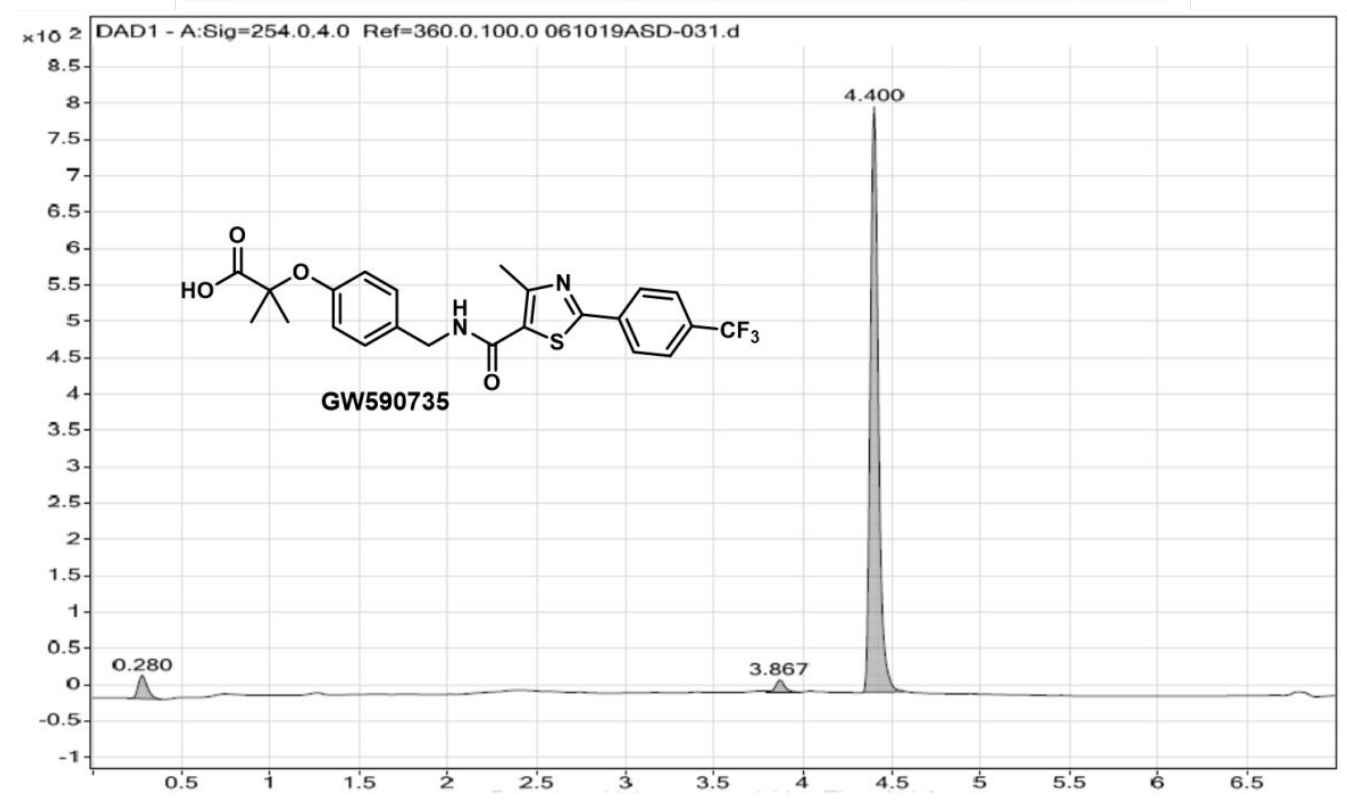

